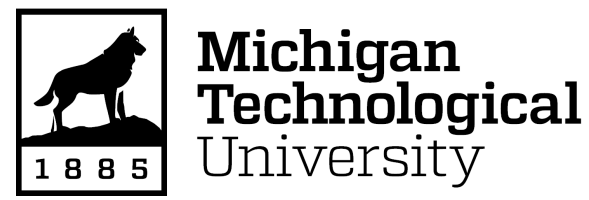

Michigan Technological University Digital Commons @ Michigan Tech

Dissertations, Master's Theses and Master's Reports

2017

\title{
The Role of Active Flow-Control Devices in the Dynamic Aeroelastic Response of Wind Turbine Rotors
}

Muraleekrishnan Menon Menon Muraleedharan Nair

Michigan Technological University, mmenon@mtu.edu

Copyright 2017 Muraleekrishnan Menon Menon Muraleedharan Nair

\section{Recommended Citation}

Menon Muraleedharan Nair, Muraleekrishnan Menon, "The Role of Active Flow-Control Devices in the Dynamic Aeroelastic Response of Wind Turbine Rotors", Open Access Dissertation, Michigan Technological University, 2017.

https://doi.org/10.37099/mtu.dc.etdr/447

Follow this and additional works at: https://digitalcommons.mtu.edu/etdr

Part of the Acoustics, Dynamics, and Controls Commons, Computer-Aided Engineering and Design Commons, and the Energy Systems Commons 
THE ROLE OF ACTIVE FLOW-CONTROL DEVICES IN THE DYNAMIC AEROELASTIC RESPONSE OF WIND TURBINE ROTORS

\author{
By \\ Muraleekrishnan M. Menon
}

\begin{abstract}
A DISSERTATION
Submitted in partial fulfillment of the requirements for the degree of DOCTOR OF PHILOSOPHY

In Mechanical Engineering - Engineering Mechanics
\end{abstract}

MICHIGAN TECHNOLOGICAL UNIVERSITY

2017

(C) 2017 Muraleekrishnan M. Menon 

This dissertation has been approved in partial fulfillment of the requirements for the Degree of DOCTOR OF PHILOSOPHY in Mechanical Engineering - Engineering Mechanics.

Department of Mechanical Engineering - Engineering Mechanics

Dissertation Advisor: $\quad$ Dr. Fernando L. Ponta

Committee Member: Dr. Leonard J. Bohmann

Committee Member: $\quad$ Dr. Lucia Gauchia

Committee Member: Dr. Rush D. Robinett

Department Chair: Dr. William W. Predebon 



\section{Dedication}

\section{To my parents, S. Padmakumari and D. Muraleedharan Nair}

who inspire me to stay truthful and dedicated in my endeavors 



\section{Contents}

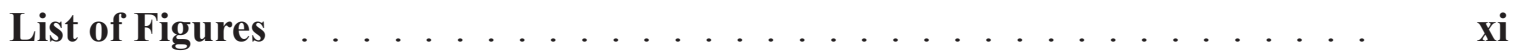

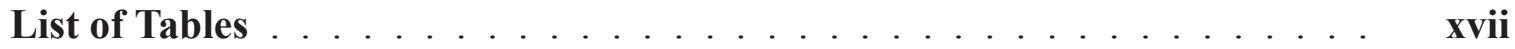

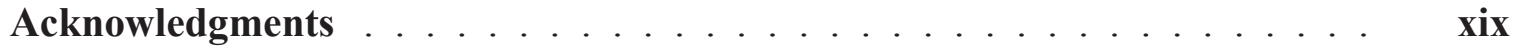

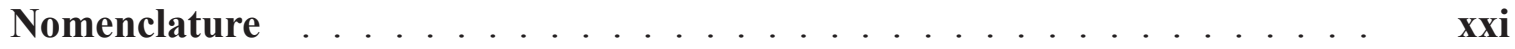

List of Abbreviations $\ldots \ldots \ldots \ldots \ldots \ldots \ldots \ldots \ldots \ldots \ldots \ldots$

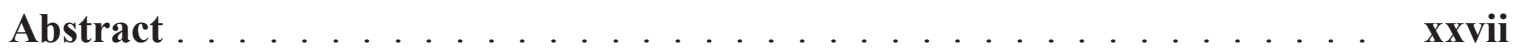

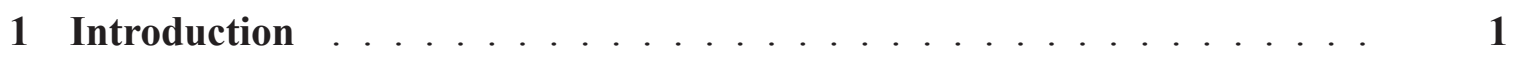

1.1 Dynamic Load Control in Wind Turbines . . . . . . . . . . . . . . 4

1.2 Aerodynamic Control . . . . . . . . . . . . . . . 5

1.3 Flow-Control Device for Load Control . . . . . . . . . . . . . 8





2 Numerical Model . . . . . . . . . . . . . . . . . . . . . . . . . . . 13 
2.1 Dynamic Rotor Deformation - Blade Element Momentum (DRD-BEM)

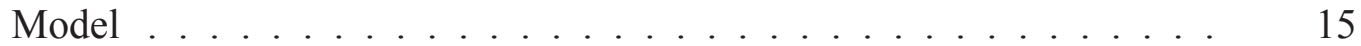

2.1.1 Blade structural model: The dimensional-reduction technique for

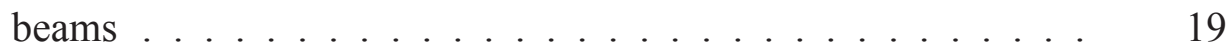

2.1.2 DRD-BEM procedure ................. 25

2.1.3 Dynamic update of corrective factors . . . . . . . . . . . 38

2.1.4 The Common ODE Framework (CODEF) . . . . . . . . . . . 42

2.2 FCD Module . . . . . . . . . . . . . . . . . . . 45

2.3 Power of Control Actuation . . . . . . . . . . . . . . 50

2.3.1 Conventional pitch-control . . . . . . . . . . . . . 52

2.3.2 Flap-actuation control . . . . . . . . . . . . . 53

2.3.2.1 Aerodynamic moment . . . . . . . . . . 54

2.3.2.2 Inertial moment . . . . . . . . . . . 56

3 Numerical Study . . . . . . . . . . . . . . . . . . 61

3.1 Need for rapid control action . . . . . . . . . . . . . . . 62

3.1.1 Aeroelastic effects of short-term load fluctuations . . . . . . . 63

3.1.2 NREL 5MW RWT as a numerical test bed . . . . . . . . . . 67

3.2 Rapid pitch control . . . . . . . . . . . . . . . . 68

3.2.1 Aeroelastic blade response to pitching control . . . . . . . . 72

3.2.2 Oscillatory blade response induced by rapid pitching action . . . 78 
3.2.3 Rotor response to rapid pitching for wind speeds above the nomi-

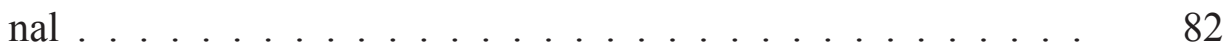

3.3 Rapid actuation of trailing-edge flaps . . . . . . . . . . 85

3.3.1 Rapid actuation of flap as dynamic load control . . . . . . . . . 87

3.3.2 Power consumption in rapid control . . . . . . . . . . . 93



4.1 Outlook for further work f . . . . . . . . . . . 106

References ......................... 107

A Copyright Agreements . . . . . . . . . . . . . . . . . . 121

A.1 Siemens press pictures . . . . . . . . . . . . . 121

A.2 Sandia report graphics . . . . . . . . . . . . . . . . . 122 



\section{List of Figures}

1.1 A Siemens SWT-7.0 shown to depict the realistic size of state-of-the-art turbine rated at $7 M W$ with a rotor of $154 m$ diameter [1]. (See appendix A.1 for copyright statement)

1.2 A $75 \mathrm{~m}$ long composite blade used on the Siemens SWT-7.0 wind turbine puts in perspective the size of turbine blades [2]. (See appendix A.1 for copyright statement) . . . .

1.3 Schematic depiction of fractional flow-control devices used for trimming control on air-

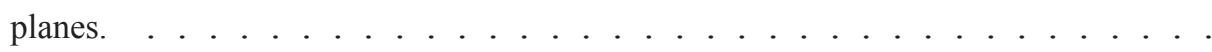

1.4 Schematic view of the trailing-edge flap and the modular attachment . . . . . . .

2.1 Schematic view of the dynamic generation of the annular actuator swept by a blade element (adaptation of a scheme presented in Burton et al. [3]). Left panel: turbine rotor with annular actuators, center panel: blade elements that correspond to the annular actuator, and right panel: a representation of Generalized Timoshenko Beam model for a generic beam section that also shows the reference-line, beam sections, and respective coordinate systems before and after deformation. . . . . . . . . . . . . .

2.2 Schematic representation of hub coordinate system according to standards from the Inter-

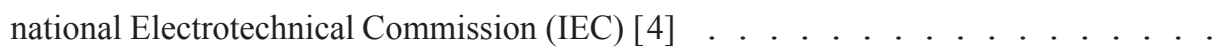


2.3 Depiction of a typical blade internal structure using a box-spar that is characterized by significant build-up of material on the spar cap zone between the shear webs. A balsa-core sandwich construction with triaxial fiberglass laminate is used in the design for exterior

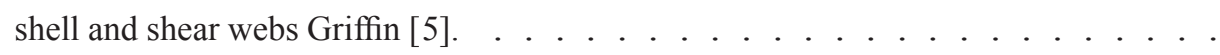

2.4 Definition of cone and tilt angles for upwind wind turbines, according to standards from the International Electrotechnical Commission (IEC) $[4] \ldots \ldots \ldots$

2.5 Blade coordinate system according to standards from the International Electrotechnical Commission (IEC) $[4] \ldots \ldots \ldots \ldots \ldots$

2.6 Graphical representation of the thrust coefficient $C T$ in function of the axial induction factor $a$. The parabolic curve given by conservation of momentum in the stream-tube; Glauert [6] and Buhl [7] empirical relations fitting Lock and Townend [8] experimental data; and the Power-Law fitting proposed here to minimize the error. The parabolic $C T$ curve form stream-tube theory is shown here affected by a tip-hub loss factor $f_{\text {th }}=0.9$ to illustrate the gap-problem on the Glauert approach. . . . . . . . . . . . .

2.7 Schematic diagram of the Common ODE Framework (CODEF) . . . . . . . .

2.8 Aerodynamic characteristics of a NACA $64_{3}-618$ attached with a $20 \%$-chord Clark Y profile trailing-edge flap actuated at configurations $\beta=-5^{\circ}, 0^{\circ}$, and $5^{\circ}$, plotted against angle of attack $\alpha$; (a) coefficient of lift $C_{l}$, (b) coefficient of drag $C_{d}$, and (c) coefficient of pitching moment $C_{m} \ldots \ldots \ldots \ldots \ldots$ 
2.9 Aerodynamic characteristics of a DU $93-\mathrm{W}-210$ attached with a $20 \%$-chord Clark Y profile trailing-edge flap actuated at configurations $\beta=-5^{\circ}, 0^{\circ}$, and $5^{\circ}$, plotted against angle of attack $\alpha$; (a) coefficient of lift $C_{l}$, (b) coefficient of drag $C_{d}$, and (c) coefficient of pitching moment $C_{m} \ldots \ldots \ldots \ldots \ldots \ldots$

2.10 Schematic representation of an NREL-5MW RWT blade, with the shaded region indicating the aerodynamically active span sections that could potentially be configured to include a Clark Y trailing-edge flap. . . . . . . . . . . . . . .

2.11 Algorithm adopted by DRD - BEM to use updated aerodynamic properties of airfoils attached with flow-control devices. . . . . . . . . . . . .

2.12 Coefficient of aerodynamic moment at the flap hinge, $C m_{h n g}$, plotted against angle of attack at the main airfoil section, $\alpha$. Left panel: NACA $64_{3}-618$, and right panel: DU 93-W-210, when attached with a 20\%-chord flap of Clark Y profile. . . . . . .

2.13 Schematic representation of airfoil-flap assembly adopted for Clark Y flaps attached on NREL 5MW RWT blades. . . . . . . . . . . . . . . . . . . .

2.14 Schematic representation of the box-beam internal structure of Clark Y flap. . . . . .

3.1 Instantaneous power generated $P$ indicative of turbine rotor behavior due to tower interference, plotted against time span of operation that includes one cycle of rotation. . .

3.2 Angle of attack $\alpha$ indicative of rotor blade behavior due to tower interference, plotted against time span of operation that includes one cycle of rotation. . . . . . . .

3.3 Schematic of a 2-D airfoil section of the blade, showing angles relevant to pitch control

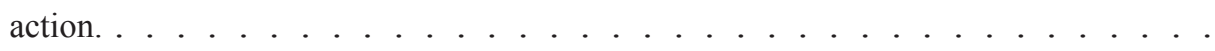


3.4 Time evolution of $\alpha$ at the $90 \%$ span section of hyper-stiff blade, for a series of pitchcontrol actions of a determined angle $\theta_{p_{c t r l}} \ldots \ldots \ldots \ldots$

3.5 Change in angle of attack, $\Delta \alpha$ for a control pitch action of $\theta_{p_{c t r l}}=5^{\circ}$ with different accelerations of pitching, $\Gamma_{\theta_{p_{c t r l}}}$; (a) $0.1 \% / s^{2}$, (b) $1 \% / s^{2}$, (c) $10 \% \mathrm{~s}^{2}$, (d) $100 \% \mathrm{~s}^{2}$. .

3.6 The $\Delta \alpha / \theta_{p_{c t r l}}$ relation in function of the pitch-control action $\theta_{p_{c t r l}}$ at different locations along the span. The dotted lines show the curves for the hyper-stiff blade, and the continuous lines the case of the standard blade. . . . . . . . . . . . . . . .

3.7 Frequency content in the aeroelastic response of the rotor for a pitch actuation of $\theta_{p_{c t r l}}=$ $5^{\circ}$ at two different accelerations of pitching; (a): acceleration of $10^{\circ} / \mathrm{s}^{2}$, (b): acceleration of $100^{\circ} / \mathrm{s}^{2}$. These spectra correspond respectively to cases (c) and (d) in figure 3.5. .

3.8 Thresholds on acceleration of pitching actuation for the appearance of single-frequency periodic oscillations, and for the appearance of multiple-frequency content. Markers $(\nabla)$ indicate the locus of cases for $\Gamma_{\theta_{p_{c t r l}}}=10^{\circ} / s^{2}$, and $100^{\circ} / s^{2}$, at $\theta_{p_{c t r l}}=5^{\circ}$, corresponding to the examples shown in figures $3.5(\mathrm{c})$ and $3.5(\mathrm{~d})$, and the frequency spectra shown in figure $3.7 \ldots \ldots \ldots \ldots \ldots \ldots$

3.9 Examples of time evolution of the angle of attack $\alpha$ for five different pitching angles, $\theta_{p_{c t r l}} \ldots \ldots \ldots \ldots \ldots \ldots \ldots$

3.10 Logarithmic decrement $\delta$, characterizing the damping at the nominal wind speed of $11.4 \mathrm{~m} / \mathrm{s}$ for different values of $\theta_{p_{c t r l}}$ (the solid line connecting the markers represents the best-fit cubic curve to the data). Also shown is the damping behavior for three wind speeds above nominal, $W_{\infty}=15,20$, and $25 \mathrm{~m} / \mathrm{s} \ldots \ldots \ldots \ldots$ 
$3.11 \theta_{p_{c t r l}(\delta=0)}$ and $\theta_{p_{(\delta=0)}}$ vs wind speed. Markers correspond to the same four wind speed cases shown in figure 3.10, and solid lines show their respective best-fit curves based on a cubic polynomial fitting. . . . . . . . . . . . . . . .

3.12 Aeroelastic response of the turbine rotor to rapid control action for flap actuation $\beta_{c t r l}=$ $-5^{\circ}$, and $5^{\circ}$ implemented in time-span of $1 s$; panels are presented to show the aerodynamic observable, deformational response, and global effects in terms of force and power. Panels (a) rotor thrust $T$, (b) instantaneous power $P$, (c) tip deflection $U_{h_{x_{t i p}}}$, and (d) angle of attack $\alpha$ at $90 \%$ span section. The case for $\beta_{c t r l}=0^{\circ}$ is shown as reference when a flap is attached but not actuated. . . . . . . . . . . . . . . . .

3.13 Consistent variation in rotor behavior for angles of flap actuation ranging between $\beta_{c t r l}=$ $-5^{\circ}$ and $+5^{\circ}$, showing an aerodynamic observable, deformational response, and global effects in terms of force and power. Panels show the final value of (a) rotor thrust $T$, (b) instantaneous power $P$, (c) tip deflection $U_{h_{x_{t i p}}}$, and (d) angle of attack $\alpha$ at $90 \%$ span section, plotted against angle of rapid flap-actuation control $\beta_{c t r l} . \quad \ldots . . .$.

3.14 Oscillatory behavior in rotor response observed for a case of $\beta_{c t r l}=-5^{\circ}$ that effects a reduction in rotor thrust $T$, at increasing rate of flap actuation - (a) $\Delta t_{c t r l}=2 \mathrm{~s}$, (b) $\Delta t_{c t r l}=1 \mathrm{~s}$ (c) $\Delta t_{c t r l}=0.2 \mathrm{~s}$, and (d) $\Delta t_{c t r l}=0.1 \mathrm{~s} \ldots \ldots \ldots \ldots$

3.15 Evolution of instantaneous power $P$ during nominal operating conditions due to rapid control action resulting in power reduction, presented for $\theta_{p_{c t r l}}=0.35^{\circ}$ on the left compared to $\beta_{c t r l}=-5^{\circ}$ cases on the right. Panels (a) and (b) show cases with $\Delta t_{c t r l}=1 \mathrm{~s}$, (c) and (d) $\Delta t_{c t r l}=0.2 \mathrm{~s}$, and (e) and (f) $\Delta t_{c t r l}=0.1 \mathrm{~s} \ldots \ldots \ldots \ldots$ 
3.16 Evolution of instantaneous power $P$ during nominal operating conditions due to rapid control resulting in power augmentation, presented for $\theta_{p_{c t r l}}=-0.2^{\circ}$ on the left compared to $\beta_{c t r l}=5^{\circ}$ cases on the right. Panels (a) and (b) show cases with $\Delta t_{c t r l}=1 \mathrm{~s}$, (c) and (d) $\Delta t_{c t r l}=0.2 \mathrm{~s}$, and (e) and (f) $\Delta t_{c t r l}=0.1 \mathrm{~s} \ldots \ldots \ldots \ldots$

3.17 Power required to perform rapid control $P_{c t r l}$, plotted in decreasing time-scale of the control application. Top panel: $\Delta t_{c t r l}=1 \mathrm{~s}$, middle panel: $\Delta t_{c t r l}=0.2 \mathrm{~s}$, bottom panel: $\Delta t_{c t r l}=0.1 \mathrm{~s}$; each panel compares rapid pitch-control against rapid flap-actuation. .

3.18 Peaks powers of control actions, $P_{c t r l}$ for comparable scenarios in rapid-pitch and rapid flap-actuation controls, plotted against the control action time span, $\Delta t_{c t r l} . \ldots 102$ 


\section{List of Tables}

3.1 NREL-5MW RWT operational parameters . . . . . . . . . . . . . 68 



\section{Acknowledgments}

It is indeed a great privilege to have worked under the guidance of Dr. Fernando L. Ponta for my doctoral research. He has been an excellent mentor, a good friend, and a remarkable source of inspiration to pursue scientific research. I am grateful for his passion and continuous support throughout the course of my degree. I would like to place on record my sincere gratitude to Dr. Ponta for serving as my academic adviser. I would like to sincerely thank my doctoral advisory committee, Dr. Lucia Gauchia, Dr. Leonard J. Bohmann, and Dr. Rush D. Robinett for their valuable inputs and interest in this research.

I am grateful to the National Science Foundation for extending financial support towards completing parts of this valuable research in science, which helps me earn this degree. I would like to thank the Department of Mechanical Engineering - Engineering Mechanics for providing me with opportunities to teach and guide undergraduate students, which also supported my educational expenses. The dedication of the departmental staff in many occasions made things easier, and I'm grateful for their support. The Doctoral Finishing Fellowship awarded through the Graduate School at Michigan Technological University was a great opportunity to focus on timely completion of the degree, and I appreciate the efforts from all well-wishers of the university in this respect.

I am extremely thankful to the WindGroup research team in the department of Mechanical 
Engineering - Engineering Mechanics for the constant support and criticisms. The numerous brainstorming sessions and philosophical discussions have shaped me into a better researcher.

As a mentor from my undergraduate days, Dr. Balakrishnan Shankar has inspired me to be a better researcher through the confidence he showed in my abilities. And, extremely important is the unrelenting support from my close-knit family and friends and their certitude, which persuades me to do better everyday. I would like to thank everyone for sticking by me through thick and thin. 


\section{Nomenclature}

$\alpha \quad$ Angle of attack of wind

$\beta \quad$ Angle of flap actuation

$\beta_{c t r l} \quad$ Angle of flap actuation as a control-action

$\delta \quad$ Logarithmic decrement

$\delta_{f l p} \quad$ Permissible design deflection on trailing-edge flap

$\theta_{p} \quad$ Angle of pitching

$\theta_{p_{0}} \quad$ Angle of initial pitch

$\theta_{p_{c t r l}} \quad$ Angle of pitch as a control-action

$\theta_{t w} \quad$ Sectional angle of twist

$\theta_{l L_{z}} \quad$ Deformation angle of twist

$\phi \quad$ Angle of incidence of flow

$\Gamma_{c t r l} \quad$ Acceleration of any control action

$\Gamma_{\theta_{p_{c t r l}}}$ Acceleration of pitch control action

$\Omega \quad$ Rotational speed

$\omega_{c t r l} \quad$ Angular velocity of control action

$\omega_{\text {str }} \quad$ Vibrational angular velocity vector of the structure

$\rho \quad$ Density 
a Axial induction factor

$a^{\prime} \quad$ Tangential induction factor

b Blade coordinate system

c Blade section chord-length

$C T \quad$ Thrust coefficient

$C_{l} \quad$ Coefficient of lift

$C_{d} \quad$ Coefficient of drag

$C_{m} \quad$ Coefficient of pitching moment

$C_{m_{h n g}} \quad$ Coefficient of pitching moment at the actuation hinge (for flaps)

C Orthogonal matrix for transformations or acting as linear operators

$\mathrm{C}_{l L} \quad$ Orthogonal matrix for transformation from the $L$ to $l$ coordinate system

$d F_{\text {lift }} \quad$ Sectional lifting force

$d F_{d r a g} \quad$ Sectional dragging force

$d M_{a e r} \quad$ Sectional pitching moment

$f_{t h} \quad$ Tip and hub loss factor

$\delta \mathbf{F} \quad$ Sectional aerodynamic force vector

$\mathbf{F}_{\text {str }} \quad$ Structural force vector

h Hub coordinate system

$I_{p_{z}} \quad$ Polar moment of inertia

$I_{t_{z}} \quad$ Translational inertia 
$k_{N} \quad$ Chord-normal stiffness

$l \quad$ Blade reference-line in instantaneous deformed configuration

$L \quad$ Blade reference-line in original non-deformed configuration

$\delta l \quad$ Length of blade element (Blade Element Theory)

$\mathbf{M}_{\text {str }} \quad$ Structural moment vector

$M_{\text {aer }} \quad$ Aerodynamic moment involved in control action

$M_{c t r l} \quad$ Moment (total) involved in control action

$M_{\text {iner }} \quad$ Inertial moment involved in control action

$P \quad$ Instantaneous rotor power

$P_{c t r l} \quad$ Power required for control actuation

$r_{h} \quad$ Radial position of the annular actuator (Momentum Theory)

$\delta r_{h} \quad$ Thickness of annular actuator at the rotor hub (Momentum Theory)

$S \quad$ Span (blade or flap)

$t \quad$ Time

$\Delta t_{c t r l} \quad$ Time-span of control action

T Rotor Thrust

$U_{h_{x t i p}}$ Blade tip displacement, axial to rotor, projected at $h u b$ coordinate system

$\mathbf{u}_{s t r} \quad$ Structural displacement vector

$\mathbf{v}_{\text {mech }}$ Mechanical velocity vector

$\mathbf{v}_{\text {str }} \quad$ Vibrational linear velocity vector of the structure 
$W_{\infty} \quad$ Free-stream wind velocity

$W_{h} \quad$ Wind velocity projected at the $h u b$ coordinate system

$W_{l} \quad$ Wind velocity projected at the instantaneous deformed configuration of the blade

$W_{\text {rel }}$ Relative wind velocity vector observed at a blade section 


\section{List of Abbreviations}

\begin{tabular}{|c|c|}
\hline BEM & Blade Element Momentum \\
\hline CFD & Computational Fluid Dynamics \\
\hline CODEF & Common Ordinary Differential Equation Framework \\
\hline DRD-BEM & Dynamic Rotation Deformation - Blade Element Momentum \\
\hline DU & Delft University \\
\hline FCD & Flow-control device \\
\hline GTBM & Generalized Timoshenko Beam Model \\
\hline HAWT & Horizontal Axis Wind Turbine \\
\hline IEC & International Electrotechnical Commission \\
\hline $\mathrm{kN}$ & kilo-Newton \\
\hline $\mathrm{kW}$ & kilo-Watt \\
\hline MW & Mega-Watt \\
\hline NACA & National Advisory Committee for Aeronautics \\
\hline NREL & National Renewable Energy Laboratory \\
\hline ODE & Ordinary Differential Equation \\
\hline RPM & Revolutions per minute \\
\hline RWT & Reference Wind Turbine \\
\hline $\mathrm{T}$ & Technical Specification \\
\hline
\end{tabular}




\section{Abstract}

The significance of wind as a renewable source of power is growing with the increasing capacity of individual utility-scale wind turbines. Contemporary wind turbines are capable of producing up to $8 \mathrm{MW}$ and consequently, their rotor sizes are rapidly growing in size. This has led to an increased emphasis on studies related to improvements and innovations in load-control methodologies. Most often than not, controlling the loads on an operational turbine is a precarious scenario, especially under high wind loading. The up-scaling of turbine rotors would thus benefit from a rationale change in load control through methodologies such as variable-speed stall, flexo-torsional adaptive blades, and active flow-control devices.

This thesis work extends the capabilities of an aeroelastic code to provide a platform to analyze wind turbines with flow-control devices as active load control techniques. It also explores the effectiveness of such devices under rapid load-control scenarios relevant to benchmark turbines. Pre-determined rapid control actions such as pitching and trailingedge flap actuation are implemented under nominal operating conditions. The benchmark turbine designed by National Renewable Energy Laboratory (NREL), which is an upwind three-bladed rotor rated at $5 \mathrm{MW}$ forms the test bed for the current thesis study. The goal is to obtain an overall understanding of the aeroelastic rotor response of utility-scale wind turbines under rapid control actions, paying special attention to the power of actuation. 



\section{Chapter 1}

\section{Introduction}

Wind is a major source of clean and sustainable energy and hence has a promising share in the future of renewable power. In Europe alone, wind accounts for $16.7 \%$ of total installed capacity (expanding from $6 \%$ in 2005), making it the second largest source of energy by capacity [9]. The increasing penetration of wind power puts more emphasis on the predictability of power production and hence, aerodynamically reliable turbine blade designs are growing in importance. The utility-scale market has been dominated by huge turbines that help to reduce the cost of energy, and heavier rotors are posing a bottleneck in expanding the capabilities of existing load-control techniques.

Utility-scale wind turbines comprises of three-bladed Horizontal Axis Wind Turbines (HAWT) installed onshore or offshore to capture the potential in higher winds. Today, 


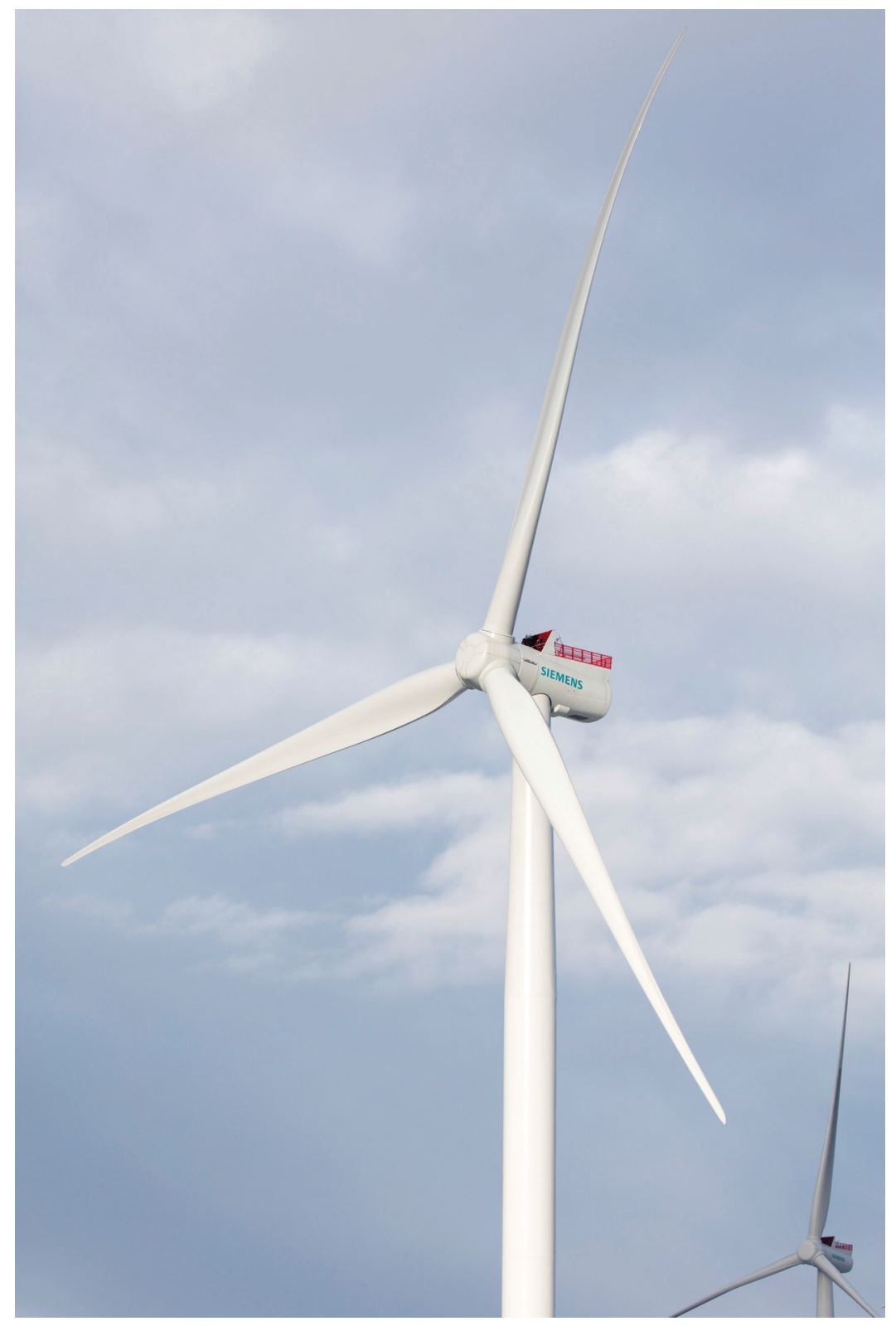

Figure 1.1: A Siemens SWT-7.0 shown to depict the realistic size of state-of-the-art turbine rated at $7 M W$ with a rotor of $154 \mathrm{~m}$ diameter [1]. (See appendix A. 1 for copyright statement)

individual wind turbines are capable of producing multi-megawatts of power. Recent studies conducted by [10] as part of the UpWind Project undertaken by European Wind Energy 
Association on huge offshore turbines indicate the capability to generate $20 \mathrm{MW}$ with rotor diameters of $250 \mathrm{~m}$. State-of-the-art turbines such as the Vestas V164-8.0 MW, are designed to generate up to $8 \mathrm{MW}$ and have rotor diameters up to $164 \mathrm{~m}[11,12,13]$. An example of most recent operational turbines installed by Siemens is the SWT-7.0-154, and is shown in figure 1.1. With rotor diameters of $154 \mathrm{~m}$, these turbines use huge blades that are up to $75 \mathrm{~m}$ long (see figure 1.2 to obtain a perspective of their proportional size). Various other operational wind turbines today have rotor diameters of more than $100 \mathrm{~m}$ [14]. Through the economies of scale factor they show a definitive trend towards upscaling of rotor size for higher power production at reduced cost. More swept area from longer blades increases the power produced due its dependence on square of the rotor diameter. These longer blades are however heavier and the down-side is presented by the cubical dependence of weight on the rotor diameter. These dependencies on rotor diameter are important in studies related to HAWT [15] and is known as the square-cubed law. Stretching the capabilities of existing load-control techniques such as pitching, stall-control, and yawing still present a bottleneck with the growing size of the turbine rotors. This has led to a deeper emphasis on studies related to improvements and innovations in dynamic loadcontrol methodologies $[16,17,18]$. 


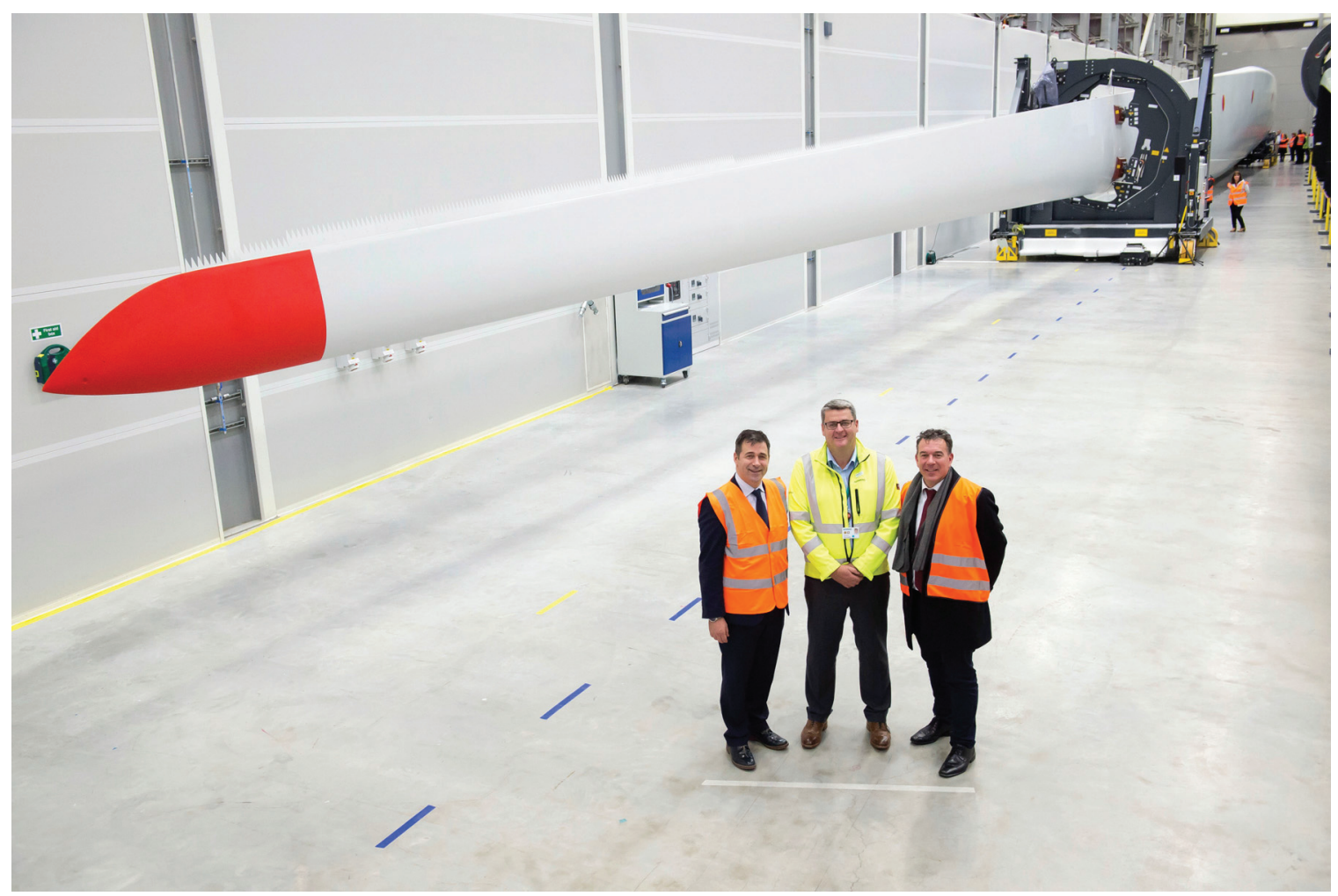

Figure 1.2: A $75 m$ long composite blade used on the Siemens SWT-7.0 wind turbine puts in perspective the size of turbine blades [2]. (See appendix A.1 for copyright statement)

\subsection{Dynamic Load Control in Wind Turbines}

Wind turbines are complex machines involving various dynamics and are subjected to fluctuating wind loads during operation. Most often than not, controlling the loads acting on an operational turbine is a precarious scenario, especially under high wind loading. Dynamic control of wind turbines alter certain aspects of the machine based on instantaneous operational state, causing a control action. The purpose varies from optimizing power generation to controlling loads acting on them and even to the extent of halting operation. The 
methodology adopted for load control can be broadly categorized into mechanical, electrical, and aerodynamic; and there is a wide-ranging study to improve specific aspects of some of these control techniques $[19,20,21,22]$. The current thesis work focus is one innovations in aerodynamic load controls.

\subsection{Aerodynamic Control}

This approach involves dynamically adapting the aerodynamics of the rotor by altering the orientation of the blade and/or rotor with respect to the wind. Methodology adopted in implementing such controls can be classified as passive and active. Passive methods make use of inbuilt characteristics such as the geometry of the turbine in an open-loop methodology. On the other hand, active load control alters specific machine attributes based on a feedback-response approach. Bianchi et al. [23] provides details of various aerodynamic load control approaches in use today. Some of the more important of these are yaw control [24, 25], stall control [26], active stall control [27], passive control using aeroelastic devices [28], and pitch control [29, 30, 31, 32, 33]. In yaw control, the entire rotor is turned in to the direction of wind, but this involves high gyroscopic loads especially in utility-scale wind turbines with huge rotors. Stall control relies on the aerodynamic stall as a result of high angles of attack achieved by the aerodynamically active sections of the blade, either as a geometric feature or by turning the blade to stall. This results in reduced power production due to a drop in lift generation. However, the fatigue loads associated 
with this state of blade operation are so high that effective load mitigation demands closer study on blade construction and materials. Active pitch control is preferred over both these approaches for next generation utility scale super wind turbines.

Control by pitch action accounts for a significant share of present day load-control methodology. In this approach, the turbine blade is rotated about its longitudinal axis to alter its orientation to wind and hence modifying the aerodynamic loads on the rotor. Most commercial turbines with pitch-control systems use a proportional-integral collective pitching approach to prevent detrimental structural loads, and limit the power generated to their rated value during high winds [34]. Under these regimes of operating condition of above rated wind speeds, the goal is to reduce the aerodynamic torque by pitching to feather and thus restricting the generated power. This also ensures reduction in the overall aerodynamic loads acting on the rotor obtained by lower angles of attack effected as a result of feathering. This ability to control the shaft torque through pitching is more beneficial compared to stall-controlled turbines that produce high stochastic loads during operation. Collective pitching can be considered useful in restricting the overall power generation at high winds, whereas individual pitching has the added advantage of mitigating cyclic loads that are more detrimental in fatigue damage of the turbines $[32,33,35,36]$. However, full span pitch controls are becoming increasingly difficult to manage with the up-scaling of turbine rotors - heavier blades need higher amounts of energy to be pitched. Lack of scalability of load mitigation studies hinders the increase in blade lengths. Today, a lot of research is underway to tackle this bottleneck to an effective control mechanism [37, 38, 39]. 


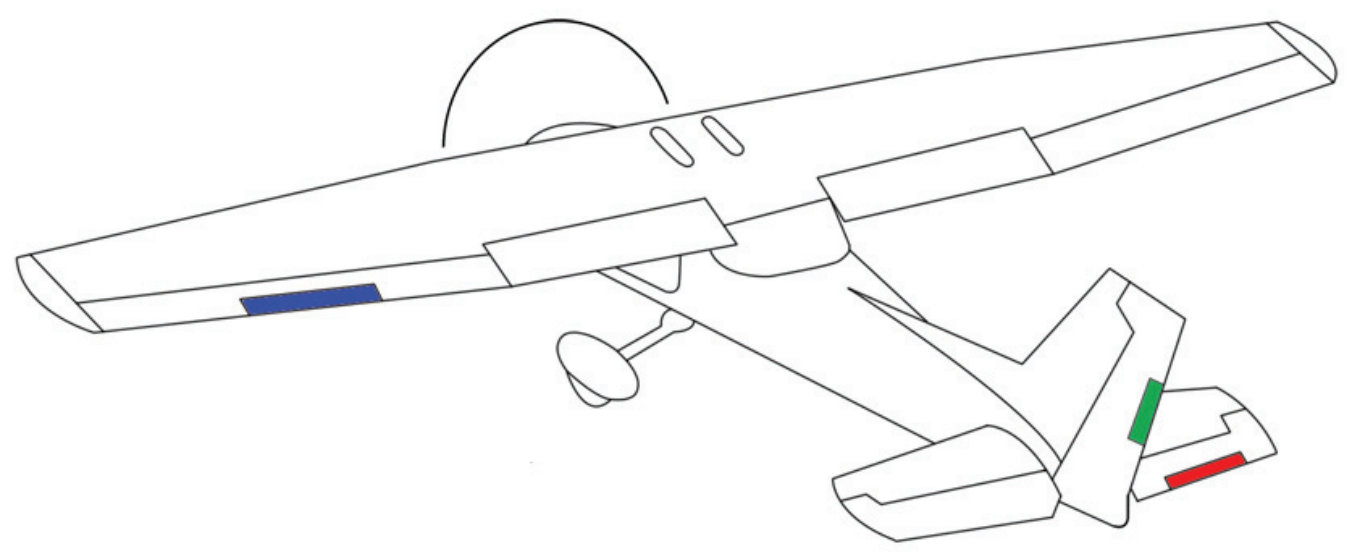

Figure 1.3: Schematic depiction of fractional flow-control devices used for trimming control on airplanes.

The up-scaling of turbine rotors would benefit from a rationale change in load control involving methodologies such as variable-speed stall, flexo-torsional adaptive blades, and active flow-control devices. Variable-speed stall machines use a control strategy combining aerodynamic characteristics of rotor blades and doubly-fed induction generation with power electronics to regulate torque, power, and reduce drive-train loads [15]. By controlling the rotor speed, turbines aim at increasing energy capture at low winds and hence maximizing power production, and limiting the rotor power to the rated output value at high winds. Such machines perform better than constant-speed stall machines in reducing extreme loads on the rotor and drive train by employing what is known as "soft stall". At high winds, the blades still operate in stall but in a more benign way [40]. However, the inertial loads acting on the rotor blades are a hold-up for up-scaling of such machines. Use of adaptive flexo-torsional blade designs are an alternative strategy [41, 42, 43, 44] where certain span sections of the blade twists as they bend under wind load, altering the angle 
of relative wind. The variable twist through the blade span passively changes the angle of attack for specific sections reducing the lift generated. However, a realistically attainable optimum twist distribution would restrict the bending-torsion coupling to the aerodynamically active part of the blade. Another strategy proposes the use of active flow control devices $[37,45]$. These devices are attachments on wings/blades capable of changing the aerodynamic behavior and are widely used in aeronautical applications. Increasing the lift generated by modifying the camber of the airfoil and/or modifying the flow around it has led these devices to be known as high-lift devices. Slats attached near the nose and tail-end attachments called flaps are examples of such devices that have been studied for use on airplane wings as early as 1914 [46, 47]. As light-weight devices, they are easier to handle and are capable of causing significant changes to the flow through minimal adjustments. Such innovative load-control approaches are less energy intensive and their relevance in future wind turbines are increasing compared to existing traditional techniques.

\subsection{Flow-Control Device for Load Control}

Flow-control devices (FCD) can be widely classified as active and passive based on the scope of fluid flow alterations. Whereas a passive device merely mixes the high momentum fluid flow and low momentum particles, an active device induces additional energy into the system. Passive devices are fixed attachments that are designed to alter flow properties in a predicted manner, and their relative position with the airfoil cannot be modified. On 
the other hand, active devices are designed to alter their configuration with changes in the flow enabling control of desired aerodynamic characteristics. Flaps and ailerons used on airplane wings are typical examples of active flow control devices. Flaps are adjustable panels near the root of the wing operated during take-off and landing to increase lift generated whereas ailerons are control surfaces towards the tip that provide lateral control for rolling. These devices have fractional chord lengths with respect to the corresponding airfoil chords and are easy to regulate [48]. Figure 1.3 depicts and example of actively controllable fractional trailing-edge devices used on airplane wings. Such devices are widely used to effect swift and minor alterations to the flight known as trimming. In the recent past, the interest in using such light weight devices on wind turbine rotor blades has been growing, and studies related to trailing-edge flaps conducted by Jost et al. [49], Wilson et al. [50], Behrens and Zhu [51], Barlas et al. [52], and Castaignet et al. [53] are significant in this context.

Flow-control devices are used on wind turbine blades either to delay stall or to regulate lift generation by modifying the fluid flow around airfoil sections $[54,55,56,57,58]$. Device shape, location on the blade, relative position with airfoil sections, and instantaneous configuration determine flow modification. Trailing-edge flaps among these devices are primarily important to the current study $[46,59]$. These external modular attachments 




Figure 1.4: Schematic view of the trailing-edge flap and the modular attachment

are designed to be regulated with low-energy actuators, enabling swift control for optimized blade performance over a wide range of wind conditions. Generating ample momentum for actuation under load ensures a cost effective and less energy intensive control approach. The structural similarities of wind turbine rotors to helicopter rotors makes studies on smart actuator systems by the European Rotorcraft Forum [60] relevant to the current study. Wind tunnel investigations conducted by the National Advisory Committee for Aeronautics (N.A.C.A.) have shown that external trailing-edge flaps act as high-lift devices and their extent of influence depends on the size, profile, hinge location, and relative angle of actuation $[61,62,63]$. Numerical studies have also shown improvement in lift behavior for multi-element airfoils derived from airfoil sections typically used for wind turbine blades [64]. Aerodynamic alterations through relative positioning can assist in load mitigation and being lighter assures faster response. Additionally, using a modular concept 
as depicted in figure 1.4 ensures minimal alteration to the manufacturing process for existing turbine blades. Such fractional chord flaps also make it easier on manufacturers for customizing these external attachments for specific blade designs. On the outlook, this approach seems to stand out compared to full-span pitching, yaw corrections, or stall control techniques. However, a comprehensive understanding of the effects of trailing-edge flaps on rotor dynamics is crucial to develop an optimum design for control.

\subsection{Focus of study}

Load control on wind turbines is widely studied and there is significant progress in understanding most of the approaches discussed above. However, studies focusing on control of rapid load variations that occurs within rotational cycles of turbine rotors are scarce. This is in spite of the fact that sources for such fluctuations like tower interference and gust loading are always a concern to the fatigue life. Even in the case of most commonly used pitch control, studies on conventional pitching to mitigate long-term variations are widespread. But there is very little progress towards studies on short-term pitch actions. Consequently, the boundaries of pitching as an effective methodology for rapid action control are not well understood. With respect to flow-control devices, the response of a flexible rotor that includes the coupled modes of deformation and aerodynamics is missing in current studies. As light weight devices they profess to have swift response and be easier to manage under dynamic operating conditions. 
This thesis work extends the capabilities of an aeroelastic code to create a platform to analyze wind turbines with flow-control devices as active load control techniques, and also explores the effectiveness of such devices under rapid load-control scenarios. Pre-determined rapid control actions such as pitching and trailing-edge flap actuation are implemented on a benchmark turbine under nominal operating conditions. The goal is to understand the aeroelastic rotor response of utility-scale wind turbines under rapid control actions, paying special attention to the power of actuation.

\subsection{Thesis outline}

This chapter provided a brief background on the significance of utility-scale wind turbines, some commonly used load control techniques, and the bottleneck in dynamic load control. Chapter 2 will discuss the details of the model used in numerical assessment of wind turbines, expansion of the control module for the integration of flow-control devices as active load control techniques, and key aspects of computing the power involved in rapid control actions such as pitching and flap-actuation. Then in chapter 3, we present the numerical results from the extensive aeroelastic analysis of a benchmark wind turbine. After a brief introduction on the need for rapid load control action on wind turbines, this chapter will explore conventional pitching and flap-actuation independently as rapid load control techniques. Finally, chapter 4 will briefly discuss the key outcomes of this thesis study on active flow-control devices for wind turbines and provide recommendations for further research. 


\section{Chapter 2}

\section{Numerical Model}

Turbine blades are highly complicated structures undergoing cyclical rotation in dynamic wind conditions. The combination of various factors such as fluctuating rotor loads, coupled aeroelastic behavior of turbine blades, interaction of blades with the tower, and a coupled control system makes the wind turbine a highly complex machine. The increasing size of the rotor and these interlinking factors make wind-tunnel studies of next generation super turbines difficult and hence necessitates full scale studies using computer models. One of the challenges in numerical study is the high computational cost involved in solving a complex non-linear 3-dimensional coupled aeroelastic problem. Numerically this problem has been resolved either as a full 3-D model or in a dimensionally-reduced manner. Full-3D simulations are computationally expensive limiting the ability to execute a wide range of cases, where different rotor designs need to be tested in various wind scenarios 
and using a range of control-strategies. Dimensionally-reduced methodology are hence preferred and employ either a vortex modeling or a stream-tube approach. The effectiveness of the model adopted also depends on its ability to alternate between the aerodynamic behavior and structural response and hence represent the coupled aspects of the turbine rotor. Reduced-order approaches typically model the structure as a Bernoulli or Timoshenko beam, either by the means of a discretization method (like finite elements) or by a modal description using limited finite number of deformation modes in the solution. The flow problem is normally solved through the well-known Blade Element Momentum (BEM) model. A combination of these two approaches allows a fully non-linear coupled scheme to represent the complexities involved (see [65] for a comprehensive discussion). Traditional aeroelastic modeling through codes such as FAST, and Aerodyn are based on this technique $[66,67,68]$. Though this approach provides a deep insight into the complex dynamics of a coupled multi-physics problem, there is a dearth in definition of the feedback introduced by the intrinsic coupling of the structural and aerodynamic modes.

The present study uses a novel numerical model capable of handling the aforementioned complexities. The current chapter introduces specific details of this numerical model, how it is implemented, and on how the control module capabilities are extended to integrate flow-control devices for active load control. Further, we will also discuss some of the key aspects related to computing power required for control actuation, which are relevant in the assessment of contemporary and innovative control techniques. 


\subsection{Dynamic Rotor Deformation - Blade Element Momen- tum (DRD-BEM) Model}

The numerical model used in this research is highly capable of representing the coupled multi-physics phenomena using two advanced numerical schemes. First, the structural response of heterogeneous composite blades is modeled to represent the complex modes of blade deformation while optimizing the computational expense [69]. Second, the flow behavior is represented using an innovative aerodynamic momentum model with capabilities to transform velocities, forces, and geometrical features through orthogonal matrices. Instantaneous deformed configuration of the rotor and their effects in computation of aerodynamic loads are completely represented in this approach known as the Dynamic Rotor Deformation - Blade Element Momentum model (DRD-BEM). This is achieved by the transformation of velocities acting at the rotor level through a series of orthogonal matrices projecting them on to the blade section, and in the same way re-projecting the forces and deformations acting at blade sections back to the rotor orientation. These numerical schemes work in the context of a multi-physics solver called the Common ODE Framework (CODEF), which also include modules that model the dynamics of the control system and electromechanic devices on the drive-train. The key features of DRD-BEM will be described in the following sub-sections, and for more details the reader is referred to Ponta et al. [70]. 
Among the stream-tube family of flow modeling for wind turbines, the Blade Element Momentum (BEM) model is widely used in the design and analysis of horizontal-axis wind turbine rotors (see [15] and [3] for further details of a classical BEM implementation). The classical formulation equates the change in momentum across an actuator disk with the aerodynamic forces computed at the blade sections using trigonometric functions to project velocities and forces. However, this is constructed on the assumption that cross-section of the blade are perpendicular to the radial axis of the actuator disk that is contained in the rotor's plane. This prevents BEM from considering various misalignments of blade sections associated with highly flexible blades during rotor operation, and hence misrepresenting the aerodynamic forces. The basics of the momentum theory remains valid and the actuator disk theory is extended for their application to horizontal axis turbine rotors. The equation of momentum changes is performed through consideration of a series of blade section elements that correspond to annular actuator rings at the rotor's plane corresponding to concentric stream-tubes. However due to blade deformations, these elemental cross-sections vary in thickness and alignment across the time-step analysis, misrepresenting the area of the annular actuator ring in momentum computation. Hence, the mathematical formulation should be able to consider the velocities projected at the instantaneous orientation of blade sections in computing the aerodynamic forces, and also use these resulting forces re-projected to the instantaneous deformed configuration of the annular actuators in the momentum equation. Additionally, recalculation of the annular actuator area needs to be performed based on instantaneous rotor deformations in equating the change in momentum 
along the stream tubes.

The DRD-BEM model used in the current study also belongs to the stream-tube family of interference models. However, a complete reformulation was adopted to take into account the misalignments at blade sections and modifications to annular actuator configurations, ensuring the resulting model fully represents the dynamic rotor deformation effects in a manner compatible with advanced structural models. This was achieved through the use of orthogonal matrices that act as linear operators in transforming the velocities and forces through a series of coordinate systems, each of which represents an important structural aspect of the rotor. The series of transformations begin at the global coordinate system aligned with the incident wind and goes through various intermediate stages culminating at the instantaneous position and attitude of the blade sections, where the axes are defined by a triad aligned along chord-normal, chord-wise, and span-wise directions. Figure 2.1 shows a schematic representation of the Blade Element Momentum model, with an annular actuator disc depicted on the left side for the corresponding blade element shown on the right side. The forces acting on the blade element of span-wise length $\delta l$ are projected on to the hub, to compute the change in momentum across the corresponding annular actuator with radial thickness $\delta r_{h}$ at a radius of $r_{h}$ from the hub center. The hub coordinate system $h$ here is defined in accordance with the International Electrotechnical Commission (IEC) [4] (see figure 2.2, and the discussion about expressions 2.10 to 2.13 in sub-section 2.1.2). Systematically equating this change in momentum ensures that the alteration to the area swept by the annular actuator is updated at every time step of the analysis. With defining 


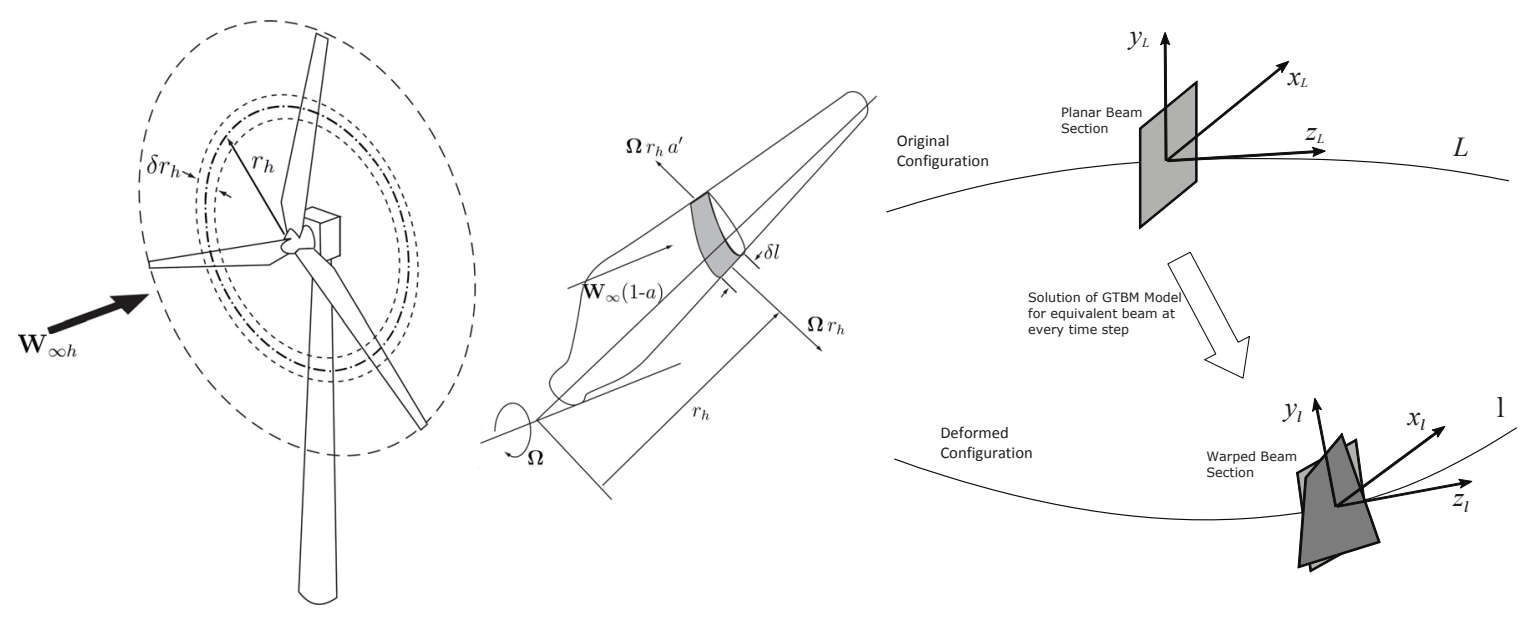

Figure 2.1: Schematic view of the dynamic generation of the annular actuator swept by a blade element (adaptation of a scheme presented in Burton et al. [3]). Left panel: turbine rotor with annular actuators, center panel: blade elements that correspond to the annular actuator, and right panel: a representation of Generalized Timoshenko Beam model for a generic beam section that also shows the reference-line, beam sections, and respective coordinate systems before and after deformation.

the hub coordinate system, $h$ at the hub of the rotor, it is also important to note the interference causes the stream-tube that is initially aligned with the direction of incident wind, deflects after passing through the annular actuator. This extend of the forces exerted on the flow (due to the presence of an actuator) will determine the amount of this deflection (see discussion about expression 2.1 in section 2.1.2). This technique allows to automatically include not only the misalignment caused by instantaneous blade deformation and/or preconforming manufacturing processes, but also the misalignments caused by the action of various mechanical devices that control yaw, pitch, and azimuthal (main shaft) rotations. Through this consistent mathematical formulation, even alterations in wind direction and changes in design characteristics such as tilt and cone angle could be accounted for in the same manner. 


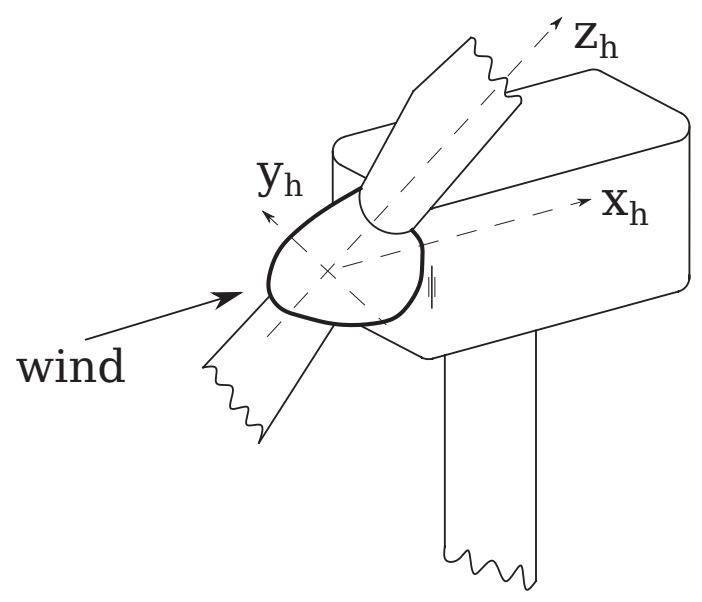

Figure 2.2: Schematic representation of hub coordinate system according to standards from the International Electrotechnical Commission (IEC) [4]

\subsubsection{Blade structural model: The dimensional-reduction technique for beams}

The numerical representation of the rotor blade structure is based on an advanced model capable of taking into account the increased flexibility of advanced blade designs. Before describing the detailed approach for DRD-BEM model, a brief description of the key features of this structural model is presented here. For a further details related to the implementation of our model and a discussions on historical background, please refer to Otero and Ponta [69] and the references therein. Otero and Ponta [69] also covers some studies conducted using the to the analyze vibrational modes of composite laminate wind turbine blades.

Reduced-order approaches to study rotor blades as slender beams amounts to substantial 


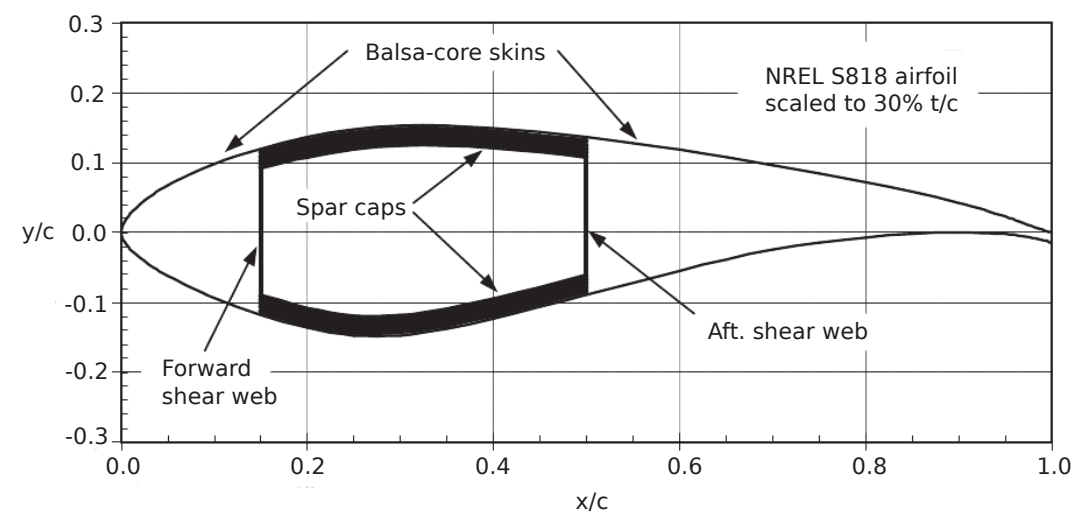

Figure 2.3: Depiction of a typical blade internal structure using a box-spar that is characterized by significant build-up of material on the spar cap zone between the shear webs. A balsa-core sandwich construction with triaxial fiberglass laminate is used in the design for exterior shell and shear webs Griffin [5].

savings in computational effort in comparison with a full 3-D analysis. However, the complex internal structure and heterogeneous distribution of material properties makes it challenging to accurately represent the complete blades characteristics using traditional approaches. See figure 2.3 from [5], for a typical example of a blade internal structure. Some of the traditional beam theories used in modeling turbine blades are the Euler-Bernoulli beam theory and standard Timoshenko beam theory. Their ad hoc kinematic assumptions however amount to intoruction of significant errors, especially when blades vibrate at wavelengths shorter than their length [71]. The Generalized Timoshenko Beam Model (GTBM) technique, used in the current study overcomes these limitations.

Originally proposed by Prof. Hodges and his collaborators [72, 73], the GTBM is a dimensional reduction technique for complex beams which may have a curved and/or twisted profile. It uses the same variables as the traditional Timoshenko beam theory, but the 
hypothesis of beam sections remaining planar after deformation is abandoned. The generalized approach takes into consideration the possibility of warping during deformation and uses a 2-D finite-element mesh to estimate the real warping of deformed sections. Through a mathematical procedure the 3-D strain energy of the beam is re-written for an equivalent one-dimensional beam in terms of the six classical variable used in traditional Timoshenko theory - the extensional strain, two transverse shear strains, the torsional curvature, and two bending curvatures. The complex geometry of blade-sections are reduced into a stiffness matrix for the the equivalent 1-D beam problem, and is then solved along the reference-line $L$, which represents the axis of the beam in its original configuration (see right panel of figure 2.1). In an asymptotic sense, the strain energy computed for the reduced 1-D model will be equivalent to the original strain energy of the 3-D blade structure.

From the numerical point of view, elimination of the ad hoc kinematic assumptions of the traditional Timoshenko theory produces a fully populated $6 \mathrm{x} 6$ symmetric stiffness matrix for the 1-D beam, instead of only the 6 individual stiffness coefficients of the traditional theory. This means that now the 6 modes of deformation are fully coupled, and it is why this technique is referred to as a generalized Timoshenko theory. Thus, bending and transverse shear in two directions, extension, torsion, and the coupled modes of deformation (like bending-torsional or bending-bending) are fully represented in a consistent theoretical frame.

Essentially, through the GTBM we are able to decouple a 3-D nonlinear elasticity problem 
into a linear 2-D cross-sectional analysis (which may be solved a priori), and a nonlinear 1D unsteady problem for the equivalent beam, which is solved using an advanced ordinary differential equation (ODE) algorithm at every time-step. The a priori 2-D analysis can be performed in parallel for multiple sections along the blade span, calculating the 3-D warping functions, and finding the stiffness matrix for the equivalent 1-D beam. Once the history of deformation for the ODE solution of the 1-D beam problem is obtained, the associated 3-D fields (displacements, stresses, and strains on the blade sections) at each time step can be recovered, a posteriori, using the 3-D warping functions calculated previously.

As can be seen in the right panel of figure 2.1, a system of coordinates intrinsic to the beam section, $\left(x_{L}, y_{L}, z_{L}\right)$, is used to represent the kinematic and dynamic variables along the original reference-line $L$. The intrinsic system follows the deformation of the beam into the instantaneous configuration $l$ to become $\left(x_{l}, y_{l}, z_{l}\right)$. When this technique is applied to blades, the intrinsic system remains aligned to the blade sections in the chord-normal, chord-wise, and span-wise directions. Thus, even during large displacements and rotations as observed in contemporary turbine blades, this technique allows accurate tracking of the position and alignment of airfoil sections as a natural outcome of the 1-D finite-element solution.

The solution of the 1-D model for the equivalent beam, as schematically indicated in the 
right panel of figure 2.1, is itself divided in two parts: dynamic and kinematic, each aspect represented through their respective set of equations (see Otero and Ponta [69] for a comprehensive description, including the complete mathematical derivations).

The dynamic part is written in terms of 4 vectorial quantities (i.e. 12 variables): linear velocity, angular velocity, force, and moments. The generalized velocity vectors represent the vibrational aspects and comprise of three variables each - 3 linear velocities $\mathbf{v}_{\text {str }}$ and the 3 angular velocities $\omega_{s t r}$ ). The generalized forces on the beam section are composed of the axial and the 2 shear forces contained in $\mathbf{F}_{\text {str }}$, plus the torsional and the 2 bending moments contained in $\mathbf{M}_{s t r}$. The 6 components of the generalized forces are directly related with the 6 variables of the Timoshenko theory through the $6 \times 6$ stiffness matrix for the equivalent beam mentioned above. The dynamic equations are essentially nonlinear, and could be either solved iteratively in a linearized mode to get steady-state solutions, or as a system of ordinary differential equations (ODEs) by means of an adaptive variable timestep ODE solver to get time dependent solutions.

The dynamic part of the solution also includes the inertia properties of the blade. Like the elastic properties discussed previously, these too are dimensionally-reduced to produce a $6 \times 6$ inertia matrix for the equivalent beam at each position along the reference-line. This matrix contains the mass per unit length, and the moments of inertia of first and second order for each blade section along the span. These are obtained from a two-dimensional integration performed over the area of each blade section which takes into account the details 
of its shape and its distribution of material properties. In this way, a full three-dimensional representation of the inertia properties of the blade are introduced into the dynamic solution. When operating in conjunction with the linear and angular velocities $\left(\mathbf{v}_{\text {str }}\right.$, and $\left.\omega_{\text {str }}\right)$, this matrix produces the 6 components of the linear and the angular momentum of the vibrational motion of the blade sections, and the inertia forces and moments associated with them. It also allows to compute the inertia forces associated with the rotation of the main shaft and the action of mechanisms like yaw or pitch. Thus, centrifugal, Coriolis, angular, and linear acceleration effects are completely accounted for in a full three-dimensional representation (see also the discussion about the computation of gravitational forces in subsection 2.1.2).

The kinematic part uses as input the previous solution of the dynamic part to produce the displacements, $\mathbf{u}_{s t r}$, and the orthogonal matrices $\mathbf{C}_{l L}$ representing the rotations of the blade sections from the original configuration $L$ to the deformed one $l$. The kinematic equations are highly nonlinear in nature due to the transcendental relations in the parametrization of rotations, and are solved through an iterative scheme, at each step of a time-dependent solution from the ODE algorithm.

Updating $\mathbf{C}_{l L}$ at every timestep of the ODE solution of the structural model, is key to transferring information between the structural and the aerodynamic models, together with the displacements of the reference-line $\mathbf{u}_{\text {str }}$, and the linear and angular vibrational velocities of the blade sections $\left(\mathbf{v}_{\text {str }}\right.$ and $\left.\omega_{\text {str }}\right)$. On the other hand, aerodynamic load information from 
the aerodynamic model is fed into the structural solution by means of the distributed aerodynamic forces due to lift, drag and the aerodynamic pitch moment on the airfoil sections.

\subsubsection{DRD-BEM procedure}

The algorithm for DRD-BEM model is a sequential process involving a series of tasks performed in thr following order:

\section{Modification of incident wind by action of the annular actuator}

First, we shall consider the velocity vector of flow passing through an annular actuator aligned with the $h u b$ coordinate system $h$. The presence of the actuator disk in the flow path causes an interference, which is measured on the flow velocity using two induction factors. A deficit in the axial velocity is determined as the effect of an axial induction factor $a$ that represents the interference in the direction normal to the actuator. And an increase in tangential velocity results from the tangential induction factor $a^{\prime}$ that represents the radial interference in a direction tangential to the rotor. Then the freestream wind is modified at the hub providing the velocity vector of wind going through the actuator as,

$$
\mathbf{W}_{h}=\left[\begin{array}{l}
W_{\infty h_{x}}(1-a) \\
W_{\infty h_{y}}+\Omega r_{h} a^{\prime} \\
W_{\infty h_{z}}
\end{array}\right]
$$


where $\mathbf{W}_{\infty h}$ is the undisturbed wind velocity field referred to the hub coordinate system, $\Omega$ is the angular velocity of the rotor, and $r_{h}$ is the instantaneous radial distance of the annular actuator (as shown in figure 2.1). As mentioned earlier, the concentric stream-tubes associated with each blade element are initially aligned with the hub coordinate system, and is deflected after passing through the actuator as a result of the forces acting on the flow particles from the actuators. The three-dimensional construction of $\mathbf{W}_{h}$ reflects this notion, reflected in the changes from $\mathbf{W}_{\infty h}$. Any changes in the orientation of incident wind due to rotor features such as a tilted rotor and/or yawing angle are represented in the $\mathbf{W}_{\infty h}$ beforehand, through a transformation from $\mathbf{W}_{\infty \text { wind }}$ with the use of orthogonal matrices. From this position, a series of orthogonal matrices are used to transform $\mathbf{W}_{h}$ through a set of coordinate systems to eventually obtain the instantaneous velocity of wind as seen by the blade element.

Orthogonal three dimensional matrices work in a twofold manner: they can act as a linear operators to transform vectors between two coordinate systems, or as mathematical representation of a rotation in the three-dimensional space (that is why they usually are simply referred to as rotation matrices). The case of coordinate transformation can be seen as a rotation of the first coordinate system to make it coincident with the second one.

The transformation of $\mathbf{W}_{\infty \text { wind }}$ into $\mathbf{W}_{\infty h}$ happens in a sequence of transformation, of which the first orthogonal matrix represents the yawing misalignment and is denoted by $\mathrm{C}_{\Delta \theta_{\text {yaw }}}$. This will take into account any misalignment between the wind direction and 


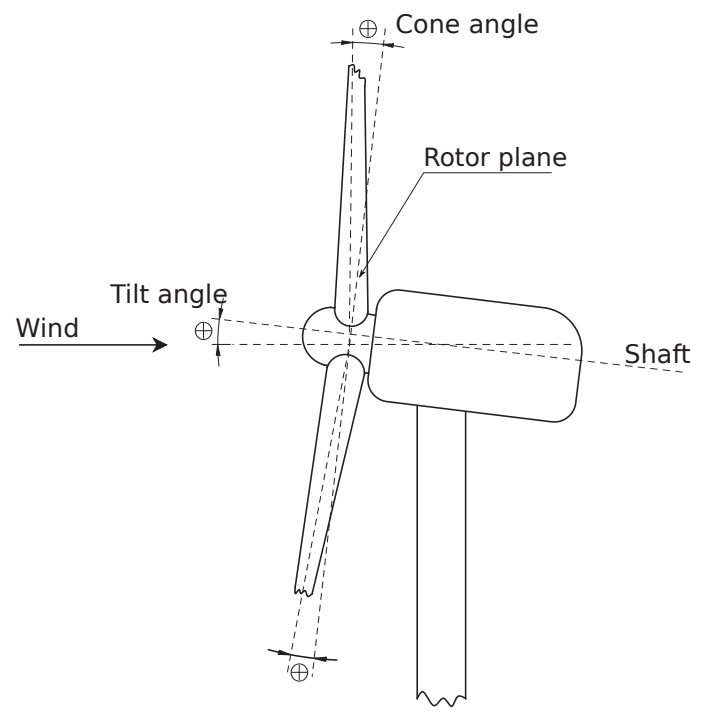

Figure 2.4: Definition of cone and tilt angles for upwind wind turbines, according to standards from the International Electrotechnical Commission (IEC) [4]

the nacelle orientation, represented by the angle $\Delta \theta_{\text {yaw }}$, analogous to a rotation around the vertical axis of the turbine. The matrix used in this transformation has the following form, resulting in the wind velocity being expressed in the nacelle coordinate system,

$$
\mathbf{C}_{\Delta \theta_{\text {yaw }}}=\left[\begin{array}{ccc}
\cos \left(-\Delta \theta_{\text {yaw }}\right) & \sin \left(-\Delta \theta_{\text {yaw }}\right) & 0 \\
-\sin \left(-\Delta \theta_{\text {yaw }}\right) & \cos \left(-\Delta \theta_{\text {yaw }}\right) & 0 \\
0 & 0 & 1
\end{array}\right]
$$

where $\Delta \theta_{\text {yaw }}=\theta_{\text {yaw }}-\theta_{\infty}$, with $\theta_{\text {yaw }}$ being the nacelle orientation and $\theta_{\infty}$ the direction of the free-stream wind. The minus sign is due to the fact that $\Delta \theta_{\text {yaw }}$ is defined positive counter-clockwise according to technical specification of IEC (TS 61400-13 IEC:2001), and both $\theta_{\text {yaw }}$ and $\theta_{\infty}$ are defined positive in clockwise sense from the North as in a compass rose. 
To obtain the wind velocity as projected at the $h u b$ coordinate system, there are two more transformations that considers the tilt orientation and azimuthal position. The vertical misalignment of turbine axis due to a tilting angle is considered as defined in the IEC standards [4] (see figure 2.4). First, a rotation around the horizontal axis of the nacelle system is contained in the tilting matrix $\mathbf{C}_{\theta_{t l t}}$. This transforms the velocity vector into a coordinate system that aligns the first axis parallel to the turbine shaft. Second, the azimuthal matrix $\mathbf{C}_{\theta_{a z}}$ transforms the wind velocity into the $h u b$ coordinate system $h$, by rotating around the main shaft to the instantaneous blade position. The $h u b$ coordinate system is depicted in figure 2.2. This results in in expression for the unperturbed wind velocity projected at the $h u b$ coordinate system:

$$
\mathbf{W}_{\infty h}=\left(\mathbf{C}_{\theta_{a z}} \mathbf{C}_{\theta_{t l t}} \mathbf{C}_{\Delta \theta_{y a w}} \mathbf{W}_{\infty \text { wind }}\right)
$$

\section{Projection of wind velocity on the blade section coordinate system}

Moving ahead from the $h u b$ coordinate system, $\mathbf{W}_{h}$ will be projected through several coordinate systems to align the velocity with the instantaneous blade section. First of these transformations are represented in the coning matrix $\mathbf{C}_{\theta_{c n}}$. This is a linear operator that takes into account the coning angle for the rotor (see figure 2.4), characterizing a rotation around the second axis of the $h u b$ coordinate system. This matrix could either represent coning as a in-built feature of the turbine as in the case of NREL-5MW reference wind turbine [74], or a control mechanism that intends to vary the coning angle 


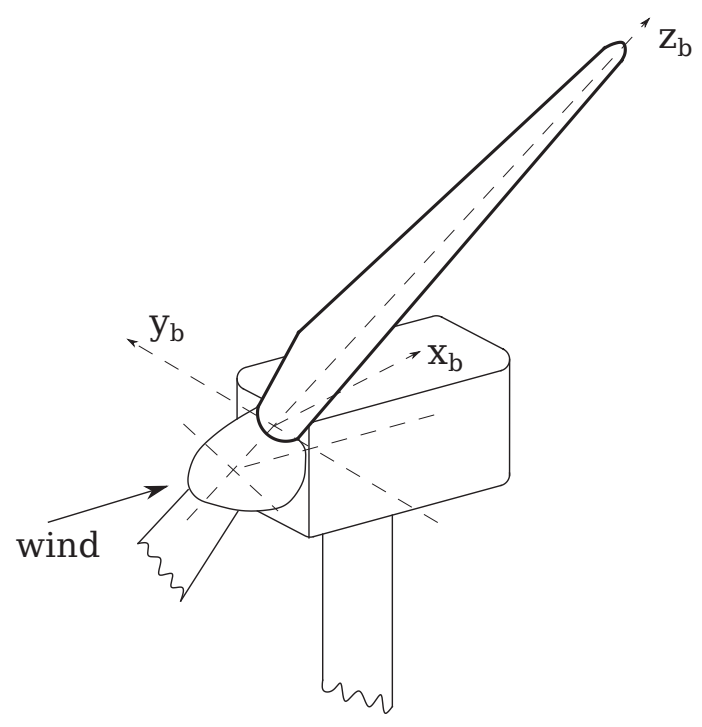

Figure 2.5: Blade coordinate system according to standards from the International Electrotechnical Commission (IEC) [4]

during operation. Either way, there will be a misalignment due to the coned rotor and the current code is designed to include both. For a detailed description of the concept of coning rotors and their effects see Jamieson [75], Crawford [76], Crawford and Platts [77].

Similarly, the pitching transformation matrix $\mathbf{C}_{\theta_{p}}$ involves a rotation around the pitch axis of the blade, which is the third axis of a coordinate system obtained from the sequence of transformations. The pitch angle $\theta_{p}$ again, could be a design feature of the turbine at various operational conditions or the result of a control mechanism, and reflects changes in pitch most often introduced by actuators of the control system. As per IEC standards [4] (see figure 2.5), we are now representing the velocity of wind at the so-called blade coordinate system denoted as $b$. As an example of these linear operators, the matrix of transformation for pitching is presented here. 


$$
\mathbf{C}_{\theta_{\mathbf{p}}}=\left[\begin{array}{ccc}
\cos \left(-\theta_{p}\right) & \sin \left(-\theta_{p}\right) & 0 \\
-\sin \left(-\theta_{p}\right) & \cos \left(-\theta_{p}\right) & 0 \\
0 & 0 & 1
\end{array}\right],
$$

with $\theta_{p}=\theta_{p_{0}}+\theta_{p_{c t r l}}$, the pitch angle, composed by $\theta_{p_{0}}$, a fixed angle set up as a constructive feature, and $\theta_{p_{c t r l}}$, the pitch angle varied by the control system. The minus sign appears here due to the sense in which positive pitch angles are defined in the IEC standards.

For turbines that use tilt or yaw as control mechanisms, a similar scheme could be used to adopt the respective rotational matrices to include the angular alterations from the control action. The interaction with control and/or electromechanical modules also requires a constant update of the matrices associated with mechanical devices. For example, in addition to the use of representing the instantaneous blade position, the azimuth matrix $\mathbf{C}_{\theta_{a z}}$ could also reflect certain control operations such as the use of variable rotor speed. Such action alter the dynamics of the electromechanical drive train for varying the rotor's angular speed $\Omega$.

The coordinate system at the instantaneous configuration of blade section represented as $\left(x_{l}, y_{l}, z_{l}\right)$ are defined along the deformed reference-line $l$ (see right panel of figure 2.1). After the pitching rotation, two more orthogonal matrices are involved in this transformation process. The first among them is based on the geometrical alignment of the blade sections along the span defined during the blade design, which could include pre-defined curvatures from bend or twist blades. Along its longitudinal axis $L$, the 
blade axis is no longer rectilinear and the third-axes of blade sections does not necessarily align with the third-axis of blade coordinate system $b$ (shown in figure 2.5). As it was mentioned earlier, the intrinsic system $L$ is aligned to the blade sections in the chord-normal, chord-wise, and span-wise directions. Thus, the above mentioned curvatures can reflect either an design twist along the longitudinal axis, or a combination of twist and pre-bending on the other two axes (i.e. coning-wise/sweeping-wise). To this end, the next orthogonal matrix $\mathbf{C}_{L b}$ represents the transformation from the blade coordinate system $b$ to the reference line $L$ of the blade in its original non-deformed configuration. This intrinsic system of coordinate for $L$ are presented as a combination of $x_{L}, y_{L}$, and $z_{L}$. The second orthogonal matrix involved in this last stage of transformation is denoted as $\mathbf{C}_{l L}$, and is provided by the solution of the kinematic equations on the structural model (as explained in sub-section 2.1.1), which transforms vectors from system $L$ to the instantaneous deformed system $l$.

These series of transformations allow the representation of the wind velocity vector $\mathbf{W}_{h}$ in the coordinate system of the blade section. At this point, we will also add the vibrational velocities $\mathbf{v}_{\text {str }}$ of the blade section (coming from the structural model), and the mechanical velocity components $\mathbf{v}_{\text {mech }}$, which are already expressed in the $l$ system. The $\mathbf{v}_{\text {mech }}$ represents motion of the blade sections due to the combined effects of mechanical devices such as yaw, pitch, and azimuthal rotations. Thus, the expression for wind velocity relative to the blade section, $\mathbf{W}_{l}$, is defined as 


$$
\mathbf{W}_{l}=\left(\mathbf{C}_{l L} \mathbf{C}_{L b} \mathbf{C}_{\theta_{p}} \mathbf{C}_{\theta_{c n}} \mathbf{W}_{h}\right)+\mathbf{v}_{s t r}+\mathbf{v}_{\text {mech }}
$$

\section{Computation of aerodynamic loads using Blade Element Theory}

Aerodynamic lift and drag forces acting at blade sections are computed using the sectional lift coefficients and the relative wind velocity. The aerodynamic coefficients are defined for each airfoil profile (at the respective sections) based on the relative angle of attack, $\alpha$. Now, with the knowledge of the magnitude of wind velocity relative to the blade section, $\left|\mathbf{W}_{l}\right|$, and its angle of attack $\alpha$, the sectional lift and drag forces per unit length of span are computed as,

$$
\begin{aligned}
& d F_{\text {lift }}=\frac{1}{2} \rho C_{l}\left|\mathbf{W}_{l}\right|^{2} c \\
& d F_{\text {drag }}=\frac{1}{2} \rho C_{d}\left|\mathbf{W}_{l}\right|^{2} c,
\end{aligned}
$$

where $C_{l}$ and $C_{d}$ are the lift and drag coefficients for the corresponding angle of attack, $\rho$ is the air density, and $c$ is the chord length of the airfoil section. The total aerodynamic load acting on the sectional blade element aligned with relative wind direction has components corresponding to the lift and drag forces and is given by 


$$
\delta \mathbf{F}_{r e l}=\left[\begin{array}{c}
d F_{\text {lift }} \\
d F_{\text {drag }} \\
0
\end{array}\right] \delta l
$$

where $\delta l$ is the span of the sectional blade element as shown in the center panel of figure 2.1.

\section{Aerodynamic forces on the hub coordinate system}

The aerodynamic forces observed at the blade section needs to be re-projected back to the $h u b$ coordinate system, which is again achieved through the use of orthogonal matrices. One important and much useful property of orthogonal matrices is that their transpose is equal to their inverse. This property is made use to transform the aerodynamic load $\delta \mathbf{F}_{r e l}$ back to the $h$ coordinate system, which is conveniently achieved by transposing the same orthogonal matrices and using them in the reverse order. Hence, the aerodynamic load on the blade element is expressed in $h$ coordinate as

$$
\boldsymbol{\delta} \mathbf{F}_{h}=\mathbf{C}_{\theta_{c n}}^{T} \mathbf{C}_{\theta_{p}}^{T} \mathbf{C}_{L b}^{T} \mathbf{C}_{l L}^{T} \mathbf{C}_{L t h a l} \mathbf{d} \mathbf{F}_{r e l} \delta l
$$

where $\mathbf{C}_{\text {Lthal }}$ is the matrix which projects the lift and drag forces onto the chord-normal and chord-wise directions, which are aligned with the coordinates of $l$. Expression (2.9) could be re-written as $\delta \mathbf{F}_{h}=\mathbf{d F}_{h} \delta l$, or in components 


$$
\boldsymbol{\delta}_{h}=\left[\begin{array}{c}
\delta F_{h_{x}} \\
\delta F_{h_{y}} \\
\delta F_{h_{z}}
\end{array}\right]=\left[\begin{array}{c}
d F_{h_{x}} \\
d F_{h_{y}} \\
d F_{h_{z}}
\end{array}\right] \delta l
$$

where $\mathbf{d} \mathbf{F}_{h}=\mathbf{C}_{\theta_{c n}}^{T} \mathbf{C}_{\theta_{p}}^{T} \mathbf{C}_{L b}^{T} \mathbf{C}_{l L}^{T} \mathbf{C}_{L t h a l} \mathbf{d} \mathbf{F}_{r e l}$.

\section{$\mathrm{v}$ Equating forces from Blade Element Theory and Momentum Theory}

Finally, the components of the force coming from the blade element theory $\delta \mathbf{F}_{h}$ are equated to the rate of change of momentum through the corresponding annular actuator. The aerodynamic force component normal to the annular actuator $\delta F_{h_{x}}$, is equated to the change in axial momentum on $W_{\infty h_{x}}$ associated with the axial interference factor $a$ (see expression 2.1), which after some algebraic modifications give

$$
d F_{h_{x}}=f_{t h} \frac{4 \pi \rho r_{h}}{B}\left(W_{\infty h_{x}}^{2} a(1-a)+\left(a^{\prime} \Omega r_{h}\right)^{2}\right) \frac{\delta r_{h}}{\delta l}
$$

where $f_{t h}$ is the combination of the tip and hub loss factors described in more detail later (sub-section 2.1.3), and $B$ is the number of blades used on the rotor. Here we included the term $\left(a^{\prime} \Omega r_{h}\right)^{2}$, which takes into account the fact that the rotation of the wake causes a pressure drop behind the actuator equal to the increase in dynamic head [3]. The term $\frac{\delta r_{h}}{\delta l}$ involves the transformation of $\delta l$ into $\delta r_{h}$, which is performed by means of the same set of orthogonal matrices already described. The tangential component $\delta F_{h_{y}}$, is then equated to the corresponding change in tangential momentum associated with induction 
factor $a^{\prime}$ which gives

$$
d F_{h_{y}}=f_{t h} \frac{4 \pi \rho r_{h}}{B}\left|W_{\infty h_{x}}\right|(1-a)\left(\Omega r_{h}\right) a^{\prime} \frac{\delta r_{h}}{\delta l}
$$

\section{Iterative solution for the induction factors}

As is observed in classic BEM approach, the set of equations (2.11) and (2.12) form a nonlinear system where the unknowns are the two induction factors $a$ and $a^{\prime}$. For each of the blade section elements, these equations are solved by an iterative process within each timestep of the aeroelastic solution. In traditional implementations of BEM, this is usually solved by functional iteration schemes starting from an initial guess value. Given the more complex nature, the DRD-BEM uses an advanced optimization algorithm to improve the stability and the speed of convergence of the iterative process. To

this end, an implicit expression for $a$ is formed from equation (2.11),

$$
a_{\text {Res }}=d F_{h_{x}}-f_{t h} \frac{4 \pi \rho r_{h}}{B}\left(W_{\infty h_{x}}^{2} a(1-a)+\left(a^{\prime} \Omega r_{h}\right)^{2}\right) \frac{\delta r_{h}}{\delta l}
$$

and equation (2.12) results in an explicit expression for $a^{\prime}$ as

$$
a^{\prime}=\frac{d F_{h_{y}} B}{f_{t h} 4 \pi \rho r_{h}\left|W_{\infty h_{x}}\right|(1-a)\left(\Omega r_{h}\right) \frac{\delta r_{h}}{\delta l}}
$$

To determine the final interference factors at each time step, the zero of equation (2.13) is solved for by minimizing the residual $a_{\text {Res }}$. This solves for the axial induction factor 
$a$, while the $a^{\prime}$ solution from equation 2.14 acts as a constraint at every time step. An adaptive algorithm based on a combination of bisection, secant, and inverse quadratic interpolation methods is used in solving the minimization problem. The main advantage of this close-interval method (instead of the traditional iteration from an initial guess value), is that the search is always bracketed between two limiting values that enclose the range where the solution is expected. It avoids the situation where the solution overshoots and diverges, or gets trapped into an endless loop. This ensures that the convergence criteria, and the error check, are constantly monitored by an efficient, and highly reliable numerical scheme [78, 79].

\section{Computation of distributed loads on the blade structure}

The dynamic loads acting on the blade structure, required by the GTBM structural part of the numerical model (see sub-section 2.1.1), are to be provided from the DRD-BEM model. These are computed as distributed loads and moments per unit span length of the blade, and are expressed in intrinsic system of coordinates at the instantaneous deformed configuration $l$. The distributed loads have two main components: one contributed from the aerodynamic forces, and the other from gravitational action.

After the deteremination of induction factors (in step VI) through convergence, the aerodynamic forces acting at each blade section are re-determined taking the interference into consideration. To this end, steps I to III are repeated but this time expressing them in system $l$. That is, $\mathbf{d F}_{l}=\mathbf{C}_{\text {Lthal }} \mathbf{d F}_{r e l}$, where the first two components give the 
chord-normal and the chord-wise aerodynamic loads. The traditional formula for computation of aerodynamic moment is used to determine $d M_{\text {aer }}$ acting at airfoil sections per unit span of the blade,

$$
d M_{a e r}=\frac{1}{2} \rho C_{m}\left|\mathbf{W}_{\text {rel }}\right|^{2} c^{2}
$$

where $\rho$ is the density of air, $C_{m}$ is the aerodynamic pitch coefficient of the airfoil section at the corresponding angle of attack $\alpha, W_{\text {rel }}$ is the velocity of relative wind at the airfoil section, and $c$ is the airfoil chord. The computation of aerodynamic forces and moment here (as well as in step III) would adopt a slightly different approach to use updated aerodynamic coefficients for blade sections, when they are fitted with flowcontrol devices. This is done through a consistent algorithm that checks for the presence or absence of such devices at every time-step, and will be discussed in more detail in section 2.2 .

The three-dimensional contribution of the gravitational action to the distributed forces and moments along the span is computed for the instantaneous position and attitude of each blade section. To this end, we use the same inertia properties included in the $6 \times 6$ dimensionally-reduced inertia matrix for the equivalent beam, previously described in sub-section 2.1.1. The numerical code has the capacity to switch the gravitational load on or off according to the preferences of the user.

With these inputs from DRD-BEM, the structural model is able to produce the dynamic 
and kinematic variables to characterize the rotor deformation. The next iteration of the process involves the inclusion of various corrective factors that are necessary in these computations.

\subsubsection{Dynamic update of corrective factors}

The implementation of the DRD-BEM also contains some additional improvements in the form of correction factors that are dynamically updated. Whereas in most traditional versions of BEM, such factors are pre-computed and remain constant along the calculation. The dynamic correction factors used in DRD-BEM aredescribed below:

$\dagger$ Airfoil aerodynamic data from static wind-tunnel tests are corrected at every timestep of the analysis, and considers both rotational-augmentation and dynamic-stall effects. The rotational-augmentation correction is based on the well-know models of Du and Selig [80] and Eggers [81]. And the dynamic-stall model is based in the works of Leishman and Beddoes [82, 83, 84]. The code also has the capacity to switch between three options for its application: it could be applied at each step of the iterative solution for the interference factors plus at the computation of the aerodynamic loads (i.e. at steps III and VII); it could be applied only during the computation of the aerodynamic loads after the iterative solution have converged (i.e. only at step VII); or it could be totally switched off. 
$\dagger$ The model is capable of using multiple data tables for the aerodynamic coefficients of the airfoil sections. These multiple data sets could be associated with different Reynolds numbers, with the static presence or active actuation of flow-control devices (such as flaps, ailerons, tabs, or spoilers), or with any other factor that modifies the original curves of coefficients versus angle of attack. The data on these tables are interpolated at every time-step providing updated coefficient values that account for the instantaneous aerodynamic conditions and/or control actions on the flow-control devices. This feature opens interesting possibilities for future studies and will be discussed in great detail in a later part of this thesis work.

$\dagger$ To ensure the availability of data for a range of angles of attack $\pm 180^{\circ}$, we use the well known extrapolation method proposed by Viterna and Janetzke [85], which is also applied in real-time like the other corrections previously mentioned (i.e. they are applied at every computation of the aerodynamic forces made in steps III and VII).

Our model also incorporates several empirical corrections that are typically present in stateof-the-art BEM models (see [3, 15]):

$\dagger$ BEM theory does not account for the influence of vortices being shed from the blade tips into the wake on the induced velocity field. These tip vortices create multiple helical structures in the wake which play a major role in the induced velocity distribution at the rotor. To compensate for this deficiency in BEM theory, a tip-loss model 
originally developed by Prandtl is implemented as a correction factor to the induced velocity field [86]. In the same way, a hub-loss model serves to correct the induced velocity resulting from vortex being shed from the blade roots at the rotor hub. Both are condensed in the $f_{t h}$ factor included in equations (2.11) to (2.14).

$\dagger$ Another modification needed in the any model based on momentum theory is the correction of the thrust on the annular actuator when operating in the so-called “turbulent-wake "state. This correction plays a key role when the turbine operates at high tip speed ratios and the axial induction factor $a$ is greater than 0.5 (in practical implementations, this limit is lowered to about 0.3 to 0.45 , depending on the corrective curve adopted). At $a=0.5$, the parabola representing the thrust coefficient $C T$ as a function of $a$ reaches its vertex (see figure 2.6), and beyond that, the basic assumptions of momentum theory on a stream-tube becomes invalid as part of the flow in the far wake that starts to propagate upstream. Physically, this flow reversal cannot occur and what actually happens is that more flow entrains from outside of the wake creating vortex structures and increasing the turbulent activity. This slows down the flow passing through the rotor, but the thrust continues to increase.

Glauert [6] was the first to propose an empirical correction to overcome this limitation in momentum theory. He fitted a parabolic function to the experimental data from Lock and Townend [8] for wind turbines operating in the turbulent wake state. Glauert's fitting function is tangent to the stream-tube $C T$ curve at $a=0.4$ (see figure 2.6). Other authors such as Burton et al. [3] and Wilson [87] also proposed 




Figure 2.6: Graphical representation of the thrust coefficient $C T$ in function of the axial induction factor $a$. The parabolic curve given by conservation of momentum in the stream-tube; Glauert [6] and Buhl [7] empirical relations fitting Lock and Townend [8] experimental data; and the Power-Law fitting proposed here to minimize the error. The parabolic $C T$ curve form stream-tube theory is shown here affected by a tip-hub loss factor $f_{t h}=0.9$ to illustrate the gap-problem on the Glauert approach.

alternative fitting functions to the experimental data. Nevertheless, a discontinuity between the fitting function and the stream-tube $C T$ function appears when correction factors for tip and hub losses are taken into account [7]. This discontinuity becomes critical when the induction factors are to be obtained by iterative approaches. Buhl [7] proposed a new empirical relationship for the thrust coefficient that solves the gap-problem by ensuring a tangent matching with the stream-tube $C T$ function regardless if it is affected by corrective factors for tip and hub losses.

The model used in this study is able to employ different empirical relations fitting the experimental data by Lock and Townend [8], that could be chosen through a switch in the input. A new corrective curve is also introduced based on a Power-Law fitting, which substantially reduces the error of approximation to Lock and Townend 
[8] empirical data and also avoids the gap-problem. This is because the Power-Law fitting always intercepts the stream-tube $C T$ function regardless of the corrective factors for tip and hub losses applied. Both, Buhl [7] curve, and our Power-Law fitting are shown in figure 2.6.

$\dagger$ The influence of the tower on the flow field around the blade must also be modeled. We use the models developed by Bak et al. [88] and Powles [89], which provide the influence of the tower on the local velocity field at all points around it. These models account for the increase in wind speed around the sides of the tower, the appearance of cross-stream velocity components, the deceleration of the flow at the stagnation zone upstream of the tower, and the velocity deficit in the separated wake behind it in case the rotor operates in a downwind configuration.

\subsubsection{The Common ODE Framework (CODEF)}

The previous sections covered the numerical approach from the perspective of interaction between the structural and aerodynamic models and how they combine to fully utilize the advanced capabilities. This is achieved through an integration of the multi-physics of these models using an ODE time-step solution. And such a platform gives the flexibility to include various other aspects that affects the dynamics of the rotor, such as the controlsystem, and electromechanical devices. 
Hitherto, we have seen how our structural model will interact with our aerodynamic model providing a comparable level of description in order to make full use of the advanced capabilities of both models. This notion of integral dynamic multi-physics modelling through an ODE solution in time could be extended to include other aspects that greatly affect the dynamics of the rotor and the overall performance of the wind-turbine, like the response of the control-system and/or the turbine's electromechanical devices.

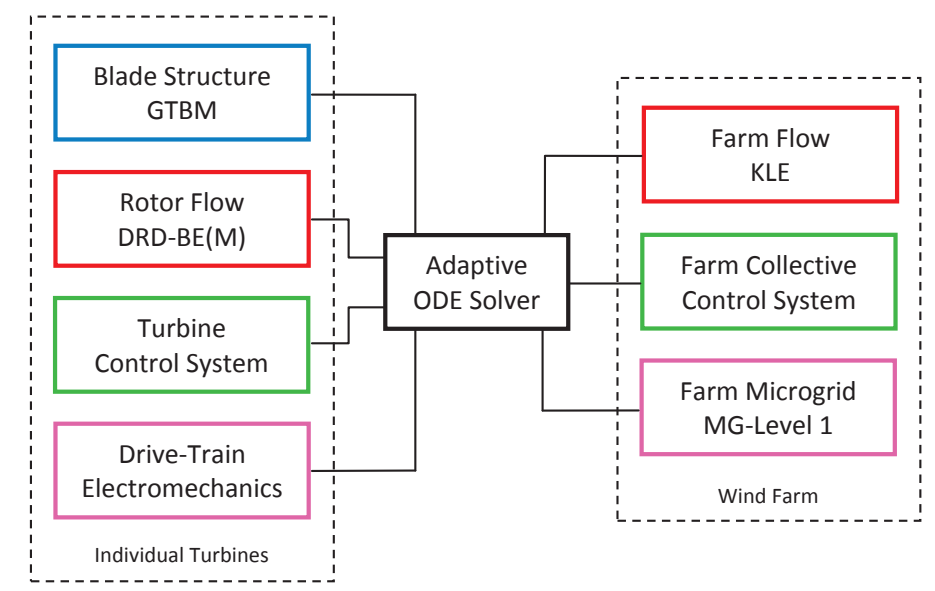

Figure 2.7: Schematic diagram of the Common ODE Framework (CODEF)

As discussed in section 2.1, the equations of motion for the 1-D finite-element problem of an equivalent beam are solved using a nonlinear adaptive ODE solver. This solver is based on a variable-timestep/variable-order ODE algorithms that monitors the truncation error at each time-step of the solution. It also attempts to minimize the truncation error that ensures stability and higher efficiency of the time-marching problem. Above all, the use of a non-linear adaptive ODE algorithm allows a natural integration of the various multi-physics aspects of the problem. Including the differential equations that model the control system and interactions with electromechanical devices allows their integration into 
the system, modifying the boundary conditions of the aeroelastic problem and vice-versa. Figure 2.7 shows a schematic diagram of this global scheme indicating the interrelation between the different modules. These modules may be treated individually, interfacing with the common ODE routine. And adopting a modular approach allows individual updates/modifications to the modules, making possible a continuous enhancement of the code in a simplified manner. Moreover, it opens the door in the future for an interconnection of the dynamics of individual turbines into an integral simulation of their collective dynamics within a wind-farm, including all physical aspects of turbine-to-turbine interaction: aerodynamic, electrical, and collective control at farm-level.

The dynamics of the control-system module and its integrated functions in the ODE framework can be extended to introduce innovative control techniques. A feedback-based approach involving the electro-mechanical components of the turbine in a closed-loop interaction with the control module is necessary for this. It involves the simulation of an integrated system of sensors, actuators, and the control devices itself, and will enable the dynamic updation of the aeroelastic, structural, and control-system properties on a realtime basis. 


\subsection{FCD Module}

In the context of this study, flow-control devices (FCD) are fractional devices on turbine blades that can be triggered to alter the air-flow dynamics near the rotor. They are also designed to dynamically change configuration during the turbine operation using low energy actuation mechanisms enabling them to actively control the flow and the resulting dynamics of the machine. Use of such devices in controls bring a two-fold advantage of the ability to vary the control parameter for a range of values while making use of minimal power to execute the control action. Fractional-chord trailing-edge flaps that can be fitted as modular attachments on to existing benchmark blades are of key interest in the current study (see figure 1.4 in chapter 1). Among other properties, flaps have the ability to either revitalize separated boundary layer or create the separation near the trailing edge of blades based on the relative angle of arrangement with the original airfoil section.

Aerodynamic loads on the blade is a cumulative effect of forces and moments acting along each section of the blade, which are given by equations 2.6, 2.7, and 2.15 (see section 2.1.2 for details). At the sectional level, these forces are primarily determined based on the nondimensional aerodynamic coefficients of lift $C_{l}$, drag $C_{d}$, and pitching moment $C_{m}$. These coefficients characterizes each airfoil section, with their values dependent on the angle of attack $\alpha$. The aerodynamic forces acting at blade sections are modified when airfoil sections are fitted with trailing-edge flaps, and the behavioral alterations depend on properties 

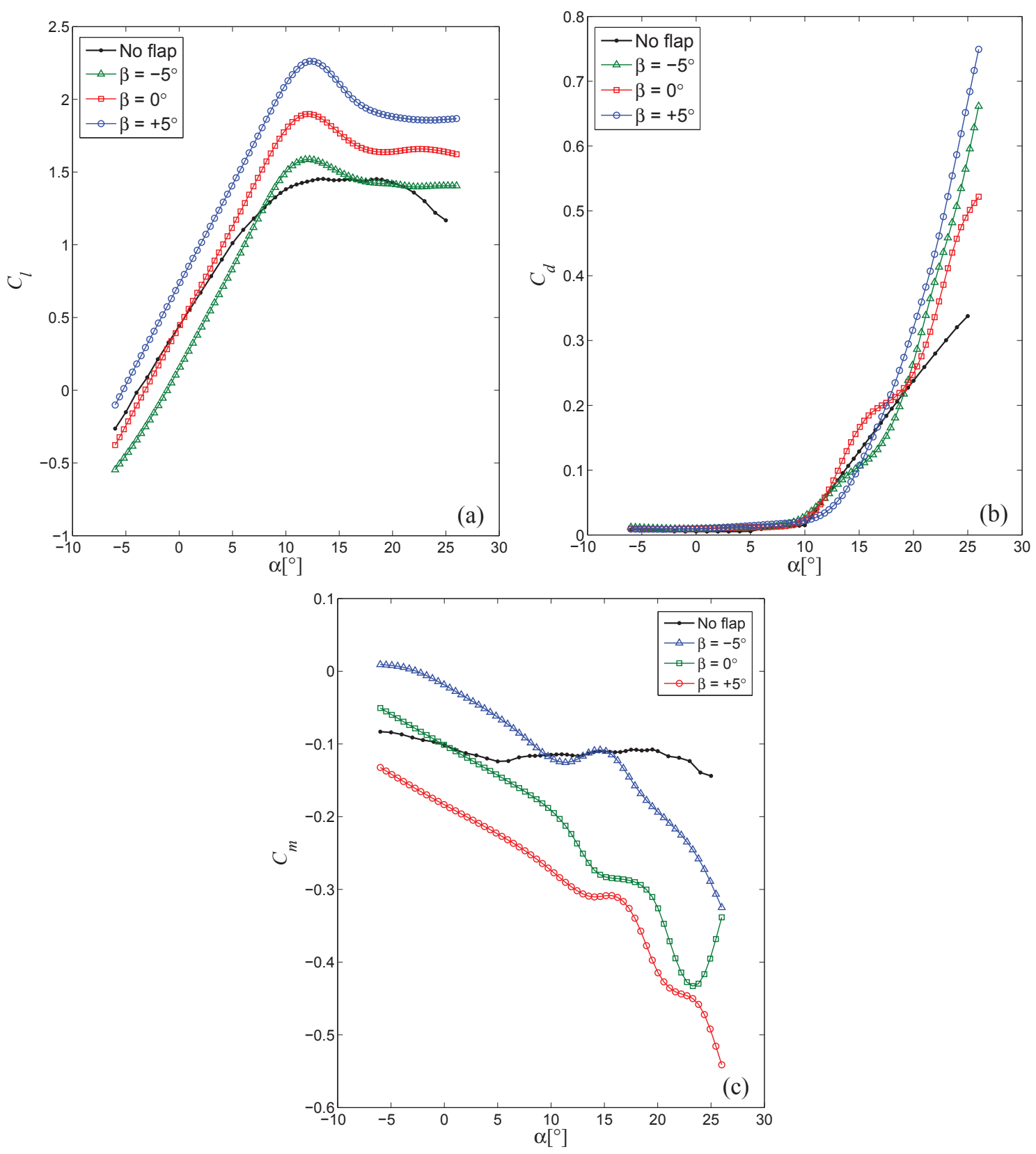

Figure 2.8: Aerodynamic characteristics of a NACA $64_{3}-618$ attached with a $20 \%$-chord Clark Y profile trailing-edge flap actuated at configurations $\beta=-5^{\circ}, 0^{\circ}$, and $5^{\circ}$, plotted against angle of attack $\alpha$; (a) coefficient of lift $C_{l}$, (b) coefficient of drag $C_{d}$, and (c) coefficient of pitching moment $C_{m}$

such as the flap chord, flap span, and the extend of flow alteration based on the airfoil-flap configurations. Fractional FCDs such as the trailing-edge flaps modifying airflow near the 
airfoil trailing-edge causing noticeable variations in the aerodynamic characteristics of the airfoil, providing a new set of aerodynamic coefficients for each configuration.
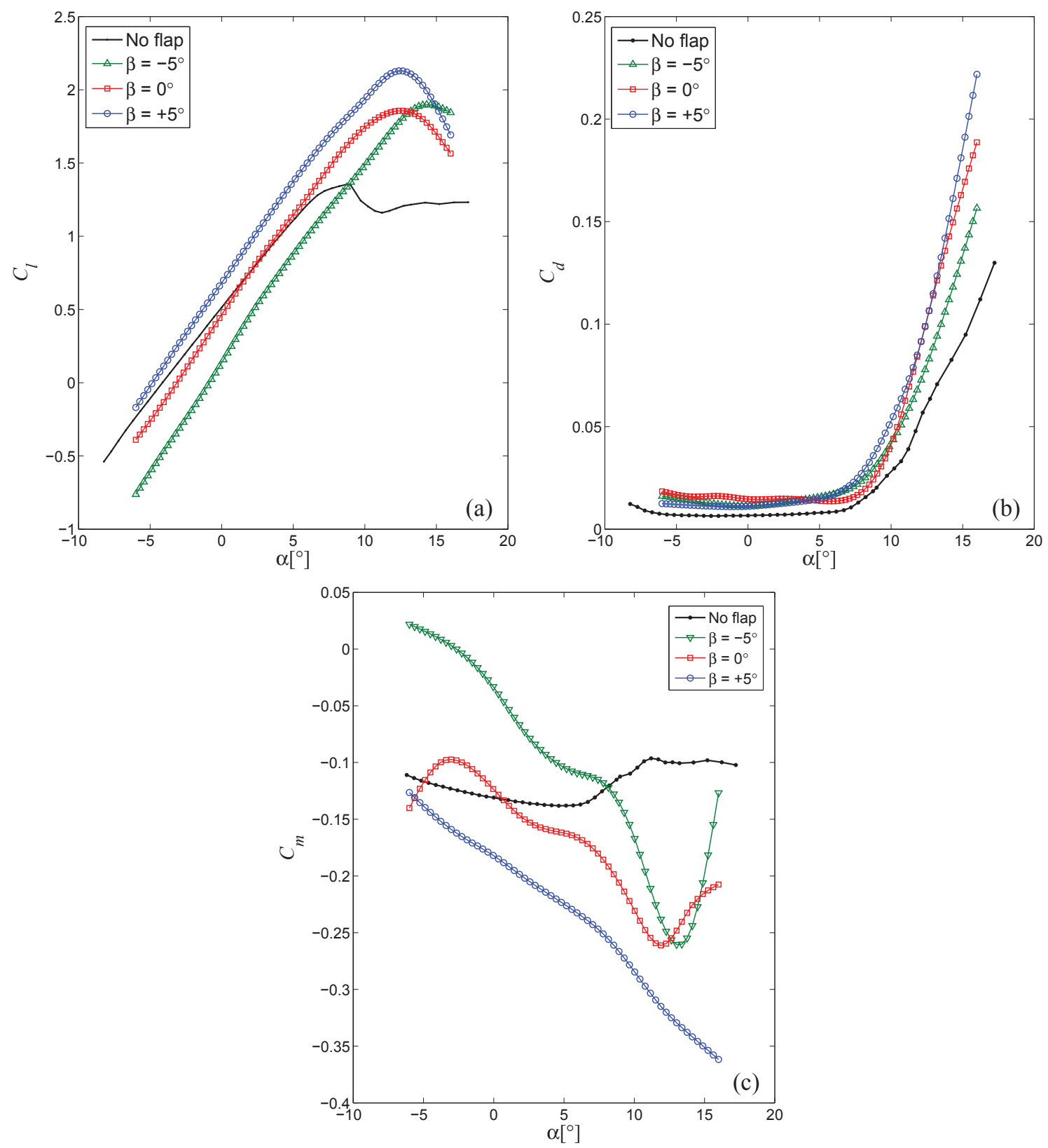

Figure 2.9: Aerodynamic characteristics of a DU $93-\mathrm{W}-210$ attached with a $20 \%$-chord Clark Y profile trailing-edge flap actuated at configurations $\beta=-5^{\circ}, 0^{\circ}$, and $5^{\circ}$, plotted against angle of attack $\alpha$; (a) coefficient of lift $C_{l}$, (b) coefficient of drag $C_{d}$, and (c) coefficient of pitching moment $C_{m}$ 
The modified aerodynamic coefficients were computed for two key airfoils NACA $64_{3}-618$ and DU 93-W-210, when attached with a 20\%-chord Clark Y profile trailing-edge flap. These airfoil sections are among the more aerodynamically efficient sections and are widely used in contemporary wind turbine blade designs, such as the benchmark wind turbine designed by National Renewable Energy Laboratory (NREL), known as the NREL-5MW Reference Wind Turbine (RWT). On such a blade, these two airfoil sections cumulatively make up about $45 \%$ of the span, as indicated in figure 2.10 . The inner regions of the blade (closer to the root) have airfoils that are thicker to ensure structural stability, whereas the outer regions (closer to the tip) use thinner airfoils that have higher aerodynamic efficiency. A major share of this aerodynamic contribution to blade operation originate from the regions shaded in figure 2.10 , which are essentially the span region equipped to be attached with trailing-edge flaps. The relative positioning of the trailing-edge flap adopted in this study will be depicted later in section 2.3. As mentioned earlier, the configuration of the airfoil-flap assembly plays a key role in determining the quantitative modification in aerodynamic behavior. These airfoil sections were studied for a range of configurations of the airfoil-flap assembly, and is defined using the relative angle $\beta$ between the airfoil and flap chords. The repository for aerodynamic characteristics of these airfoil section are available for a range of $\beta=-5^{\circ}$ to $\beta=5^{\circ}$, evaluated at regular intervals of $\beta$ configurations. Figure 2.8 shows the non-dimensional sectional coefficients of lift, drag, and pitching moment characterized for NACA $64_{3}-618$, and figure 2.9 shows the same for DU 93-W-210. Aerodynamic coefficients of these two key airfoil sections 


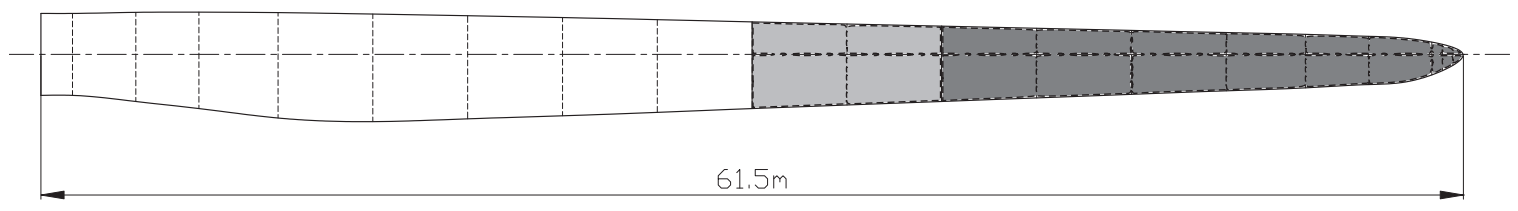

Figure 2.10: Schematic representation of an NREL-5MW RWT blade, with the shaded region indicating the aerodynamically active span sections that could potentially be configured to include a Clark Y trailing-edge flap.

with attached trailing-edge flaps present valuable data for the design of future innovative turbine blades with active flow- control devices. These properties were obtained from an extensive study on two-dimensional flow characterization for a wide range of airfoils-flap assemblies. A steady-state pressure-based computational fluid dynamic solver was used to this effect, and the range of flap configurations cover a substantial set of scenarios relevant to wind turbine operating conditions. For more details of this study, the reader is referred to $[90]$ and the references therein.

The control system module in CODEF currently has the ability to integrate the dynamics of control techniques such as yaw, pitch, and coning. Using trailing-edge flaps as a prototype, the module is extended with the capability to incorporate the dynamics of flow-control devices (FCD), simulating the interaction of such control actions with the dynamic aeroelastic response of the rotor. This means that the effects on the rotor dynamics from a control decision of the FCD module and vice-versa will be evaluated at every instant of the dynamic numerical simulation. The aerodynamic characteristics of modified airfoil sections fitted with fractional trailing-edge flaps are made available to the control module, which has a functionality to interpolate the coefficients from the repository based on the instantaneous 
configuration. At each instant of the time-step analysis (see section 2.1.2), based on the instantaneous input to the control module, the adaptive algorithm evaluates the presence or absence of a trailing-edge flap for each section of the blade. As a result, each blade section adopts either their original aerodynamic characteristics (in the absence of FCD) or accept an updated set of values (in the presence of FCD). The flowchart shown in figure 2.11 gives an overview of the functional algorithm that is used by the control system module of CODEF. The aerodynamic coefficients and the resulting loads acting at each airfoil sections will now depend on two instantaneous parameters - angle of attack $\alpha$ at the blade section, and angle of flap actuation $\beta$ defining the airfoil-flap configuration. The adaptive ODE framework ensures that structural deformations and their effects on the aerodynamic loads that arise as a result of such aerodynamic alterations, are also considered through the natural integration of the multi-physics of the machine.

\subsection{Power of Control Actuation}

For a comparative evaluation of innovative load-control techniques in rapid response situations, an aeroelastic analysis is performed on a benchmark wind turbine. Conventional pitch-control and flap-actuation control are independently implemented on pre-determined load-control scenarios. The dynamic response of the rotor is assessed for the entire time of operation and the power needed for performing the control action $P_{c t r l}$ is computed. This section will discuss the analytical approach use to determine $P_{c t r l}$ for both pitching 


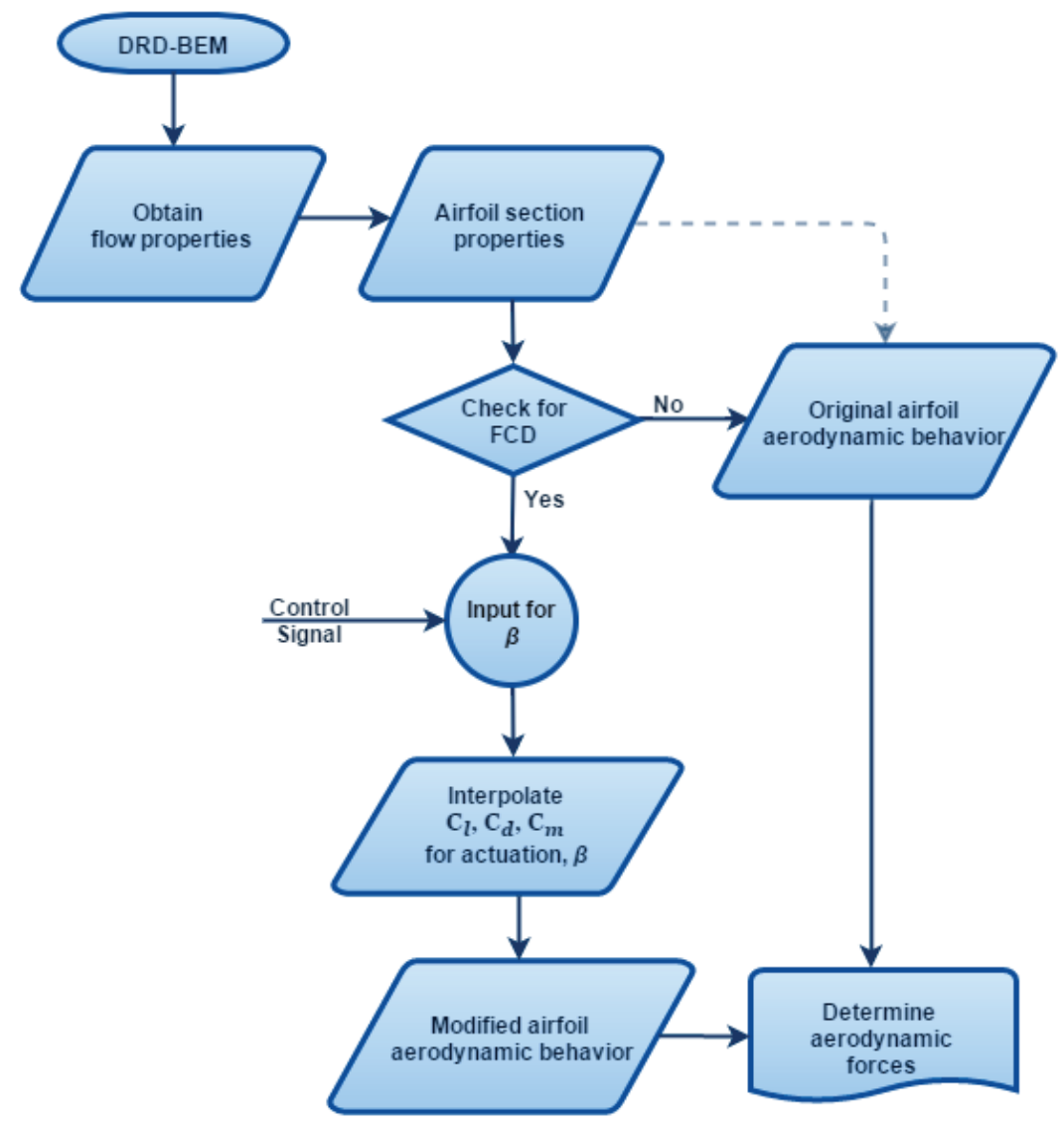

Figure 2.11: Algorithm adopted by DRD - BEM to use updated aerodynamic properties of airfoils attached with flow-control devices.

and flap-actuation control approaches from the numerical results of the aeroelastic analysis.

The power required for control action is determined at every instant of the turbine operation and is computed as

$$
P_{c t r l}=M_{c t r l} \omega_{c t r l} .
$$

where $M_{c t r l}$ is the total control moment, and $\omega_{c t r l}$ is the angular velocity of the control 
action. Here, the control moment is defined as the total moment required to effectuate a control action that involves overcoming the instantaneous aerodynamic moment, $M_{a e r}$, and the inertial moment $M_{\text {iner }}$ of the control device. In the case of pitch-control the device is entire blade while only the fractional-span flap is considered as the device in flap-actuation control.

\subsubsection{Conventional pitch-control}

Conventional pitch-control action involves rotating the blade around its longitudinal axis $(L$ in the original configuration, and $l$ in the deformed one), which is considered as the pitching axis. Physically, the pitch control is achieved through hydraulic actuators or electric motors, and hence the power supplied to these actuators become relevant in the equation for total generated power of the turbine. In case of pitching, $P_{c t r l}$ will depend on both the instantaneous aerodynamic loads and the inertia of the entire blade. The sum of instantaneous blade root moment for each blade around their respective pitching axes, obtained from the rotor simulation gives the total moment needed to pitch one blade. In the rapid control scenarios studied, a cumulative pitching of all three blades is implemented, and the total control moment $M_{c t r l}$ is obtained as the sum of moments involved in these operations. This $M_{c t r l}$ value is directly obtained as a result of the aeroelatic analysis that provides the loads acting at the blade roots at each time-step of the turbine operation. The other significant component in the $P_{c t r l}$ calculation is the rapidity of control action, which in this 
case would be the velocity of pitching $\omega_{\theta_{p_{c t r l}}}$. As the control scenario is pre-designed, the rapidity of actuation is available for calculations from the beginning. The equation 2.16 is then used to determine the instantaneous power required for the pitch-control action.

\subsubsection{Flap-actuation control}

Flap-actuation as an active rapid-control action involves altering the airfoil-flap configuration by energizing the flap from one configuration to another. As in the case of pitchcontrol, a coupled effect of aerodynamic and inertial loads acting on the flap impact the power needs $P_{c t r l}$ of the control action. However, the computation of $P_{c t r l}$ in this case is slightly different and will be done independently for overcoming the aerodynamic moments and inertial moments that are involved. As these FCDs are much lighter than the blade itself the inertial effect on the rotor dynamics are negligible and hence, the control module of CODEF integrates only the aerodynamic effects of trailing-edge flaps in the aeroelastic analysis. Computation of $P_{c t r l}$ is effectively based on the moment required to overcome both the aerodynamic and inertial loads at every instant of operation. However, the computation of aerodynamic moment, $M_{a e r}$ and inertial moment, $M_{\text {iner }}$ are done separately, considering the instantaneous aerodynamic loads and the inertial loads acting on the flap hinge respectively. The rapidity of control action is defined by the velocity of flapactuation $\omega_{c t r l}$, which is known from the design of the load-control scenarios. The total moment required for the control actuation is given as $M_{c t r l}=M_{a e r}+M_{i n e r}$, which is then 
used in equation 2.16 along with the rapidity of flap actuation to obtain the power required for flap-actuation control. The factors involved in the computations of $M_{\text {aer }}$ and $M_{\text {iner }}$ will be discussed in the following sub-sections.

\subsubsection{Aerodynamic moment}

Aerodynamic loads acting on a turbine blades primarily depends on the instantaneous flow characteristics faced by the rotor such as the velocity of wind, rotor orientation, and rotational speed. The forces and moments acting on an attached trailing-edge flap depends also on the configuration of airfoil-flap assembly (provided by flap-actuation angle $\beta$ ), and the instantaneous angle of attack $\alpha$ observed at the respective blade sections. To enable a flap actuation in the direction desired by the control action, the actuation mechanisms should supply enough torque to overcome the aerodynamic loads acting at that instant. The most important information necessary to compute this aerodynamic torque (and hence the power required) is the coefficient of aerodynamic moment around the flap actuation hinge, denoted by $C_{m_{h n g}}$. This coefficient value depends on various factors such as the airfoil-flap configuration, instantaneous angle of attack $\alpha$, and the airfoil profile itself. Figure 2.12 presents coefficients of hinge moments computed for both NACA $64_{3}-618$, and DU 93-W-210 airfoil sections with an attached 20\%-chord Clark Y profile trailing-edge flap, for a range of configurations relevant to the current study. These results were obtained from an extension of the two-dimensional computational fluid dynamic study on airfoil-flap 

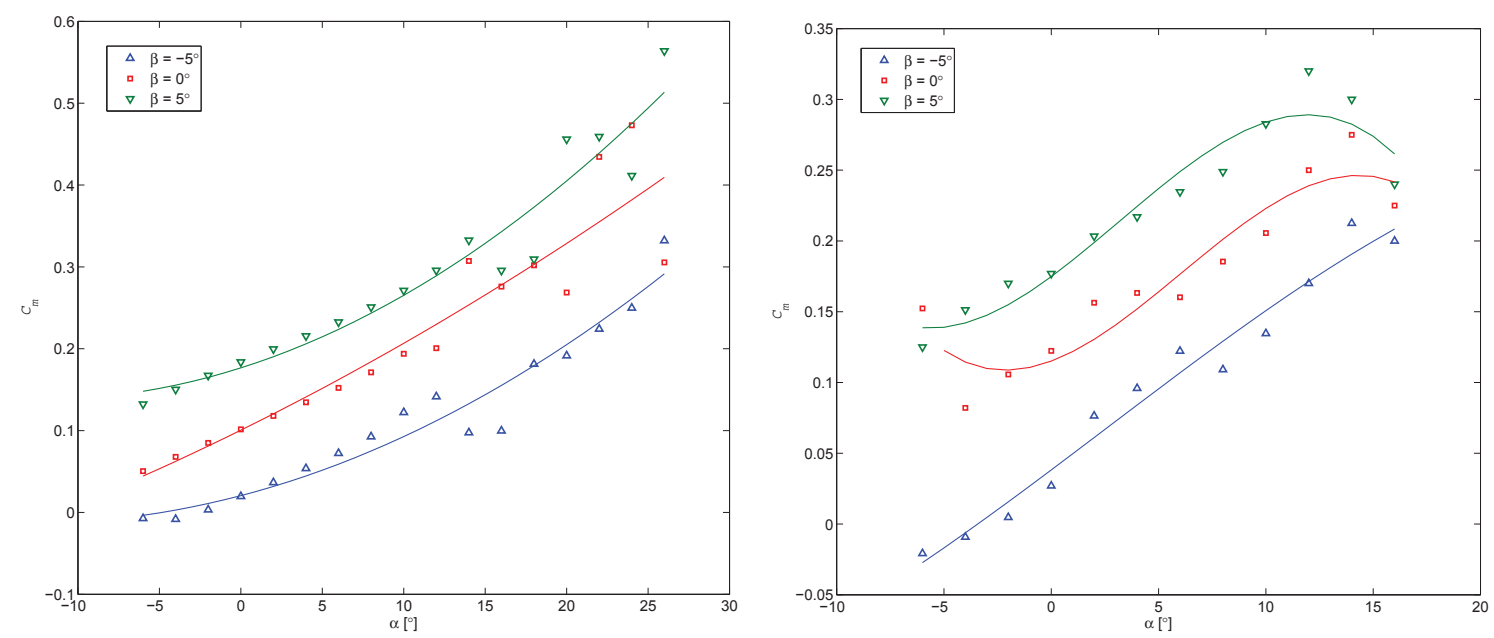

Figure 2.12: Coefficient of aerodynamic moment at the flap hinge, $C m_{h n g}$, plotted against angle of attack at the main airfoil section, $\alpha$. Left panel: NACA $64_{3}-618$, and right panel: DU 93-W-210, when attached with a $20 \%$-chord flap of Clark Y profile.

assembly (see section 2.2). The converged turbulent flow solutions were used to compute the normalized coefficients of moment around the flap hinge, at specific flap actuation angles and for the range of $\alpha$ relevant to wind power applications. These coefficients of hinge moment, $C_{m_{h n g}}$ form a repository of normalized 2-dimensional characteristic of an active trailing-edge flap that can be configured across the span of the turbine blade. As discussed in section 2.2, these two airfoil sections together cover a range of about $45 \%$ span of the NREL 5MW RWT blade, providing a strong platform to assess wide-ranging scenarios for flap actuation. Characteristics of the modular trailing-edge flap and the instantaneous configurations are available during design of the load-control scenarios. The instantaneous $C_{m_{h n g}}$ value is obtained from the corresponding configuration, and the aerodynamic moment is computed as 


$$
M_{\text {aer }}=\left(\frac{1}{2} \rho c^{2} W_{r e l}^{2} C_{m_{h n g}}\right) S .
$$

where $\rho$ is the density of air, $c$ is the design chord length of the trailing-edge flap at the blade section, $W_{\text {rel }}$ is the magnitude of instantaneous relative velocity of wind observed at the blade section, $C_{m_{h n g}}$ is the instantaneous coefficient of aerodynamic moment around the flap-actuation hinge, and $S$ is the total span of the trailing-edge flap.

\subsubsection{Inertial moment}

The moment around the flap-actuation hinge due to inertia consist of two components translational, $I_{t_{z}}$ and polar moment, $I_{p_{z}}$. The translational part is computed as $I_{t_{z}}=m r^{2}$, where $m$ is the total mass of the flap and $r$ is the distance from the point of load concentration to the actuation hinge. This study pertains to Clark Y profile trailing-edge flap that has a reference line defined along the span at $37.5 \%$ of the chord, measured from the leading-edge. The position of the flap-actuation hinge is adopted from an extensive experimental study for optimization of Clark Y profile flap positioning on high-lift airfoils such as NACA 23012 [61]. Figure 2.13 shows a schematic of the airfoil-flap assembly that presents the relative location of flap-actuation hinge with distances marked in proportion of the chord lengths. The total sectional aerodynamic loads are considered to be concentrated along the reference line, which for the current study is defined at $12.5 \%$ behind the 


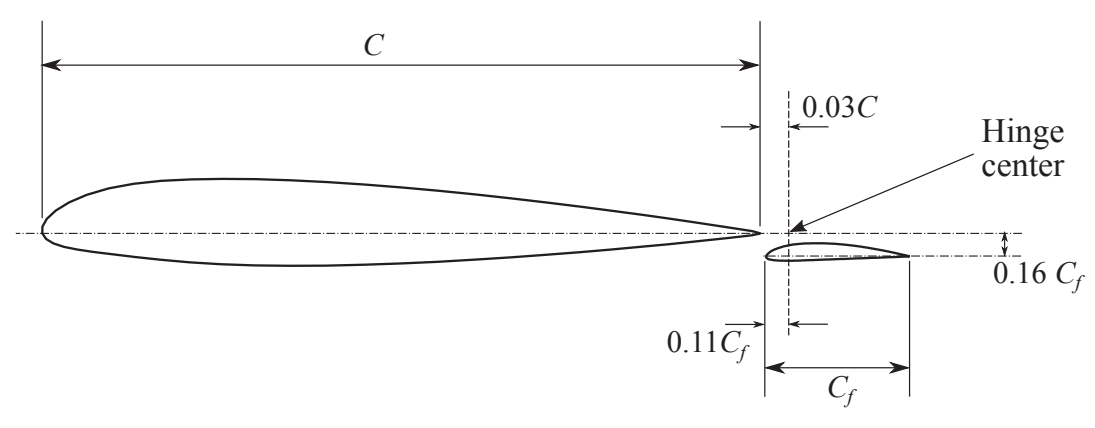

Figure 2.13: Schematic representation of airfoil-flap assembly adopted for Clark Y flaps attached on NREL 5MW RWT blades.

quarter-chord length i.e. at $37.5 \%$ of the flap chord. Hence the distance $r$ is calculated from this reference line to the flap-actuation hinge (as defined in figure 2.13).

The internal structure of the trailing-edge flap is designed in one of the most common approaches found on turbine blade designs, using the box-beam-spar ideology. A schematic representation of the internal structure developed for Clark Y is presented in figure 2.14. This internal structure is necessary to withstand the continued aerodynamic loads during turbine operation, and the total mass of the flap is determined primarily from these manufacturing considerations. The intended use of flaps as fractional-chord devices and to be attached on shorter spans of the blade, allows the design of strong internal structures without significantly adding weight. As these flaps are attached as modular devices (see figure 1.4 in section 1.3) on the blade, the desired aerodynamic alterations are effective only span-wise deflections are minimal. For NREL 5MW RWT turbine blades with Clark Y 


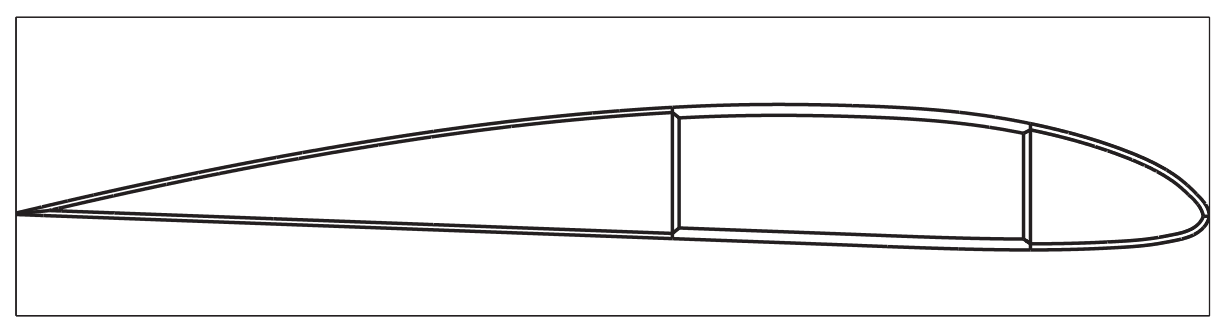

Figure 2.14: Schematic representation of the box-beam internal structure of Clark Y flap.

flaps, the span-wise (or chord-normal) deflection was designed to be $5 \%$ of the distance between the trailing-edge of airfoil and nose of the flap in a configuration of $\beta=5^{\circ}$. Higher span-wise deflections stand the risk of altering the design gap beyond their original configuration, causing a misrepresentation of the modified aerodynamic properties being used for the respective configurations. Considering uniform aerodynamic loading along the span of an attached flap, the chord-normal stiffness was computed using the equation 2.18 for uniformly loaded beams. Due to their higher significance in aerodynamic span-wise bending, forces in the chord-normal direction were used to determine the chord-normal stiffness for a permissible bending deflection.

$$
k_{N}=\frac{5}{384}\left(\frac{w S^{4}}{\delta_{f l p}}\right) .
$$

where $k_{N}$ is the chord-normal stiffness of the flap considered as a uniformly-loaded onedimensional beam, $w$ is the uniform aerodynamic load acting on the flap, $S$ is flap span, and $\delta_{f l p}$ is the design deflection permissible on the flap such that the aerodynamic effects of the airfoil-flap assembly is not lost. A series of internal structures are numerically designed for 
the flaps to obtain a matching value of the design chord-normal stiffness $k_{N}$. A box-beamspar internal structure designed for Clark Y profile (shown in figure 2.14) provided the sectional inertial properties including the mass per unit span length, chord-wise stiffness, span-wise stiffness, and polar moment of inertia. The flap span from load-control design is used in determining the total mass of the flap, which in turn provides the translational inertia, $I_{t_{z}}$. The inertia of the flap around itself defined as the polar moment of inertia, $I_{p_{z}}$ is relevant to the final computations and is also obtained from the internal structure design. Finally, the total inertial moment acting on the flap is computed as

$$
M_{\text {iner }}=\left(I_{t_{z}}+I_{p_{z}}\right) \Gamma_{c t r l}
$$

where $\Gamma_{c t r l}$ is the acceleration of flap-actuation during the control action. Having started the computation from the sectional properties of the flap profile, the value of $M_{\text {iner }}$ represents the total torque/moment required to overcome the inertial loads acting on the entire span of the re-dimensionalized trailing-edge flap. As described earlier in this section, this will contribute to the computations of instantaneous power required for control-actuation. 



\section{Chapter 3}

\section{Numerical Study}

This chapter presents the results of numerical experiments conducted on a benchmark turbine to study the dynamic aeroelastic effects on the rotor due to rapid control actions. The methodologies studied are collective full-scale pitching of all blades and dynamic actuation of fractional trailing-edge flap, which are implemented independently leading to comparable global changes on the rotor. The first section covers the significance of control actions designed for counteracting rapid load fluctuations and introduces the benchmark turbine used in assessing such scenarios. In the second section, we shall look at the dynamic effects on the rotor when subjected to pre-determined pitch-control scenarios designed for rapid action. The response of the rotor in nominal operating conditions will provide insight into rapid-action of cumulative pitching and their limitations, and assessment of the rotor in above rated wind speeds furthers these observations. The third section discusses the 
effects of rapid flap-actuation control on a modified rotor with trailing-edge flaps attached along $10 \%$ span of the blade, in comparable loading scenarios. In addition to understanding the dynamic response of such a rotor in limited actuation range, this will give a deeper insight into the structural limitations of rapid control actions turbines with flexible blades. In addition, this section also establishes the advantages of flow-control devices over pitching in rapid action scenarios through a comparative study of the power needed for control actuation when implemented in short time-scales.

\subsection{Need for rapid control action}

Load control is an integral part of any operational wind turbine, and the purpose varies depending on the situation to be handled. More often than not, dynamic controls alter the instantaneous state or operational regime to counter-act wind load variations that are sustained for a long period. For example, turbines based on the variable-speed concept have rotor speeds assigned for specific velocities of wind above and below the nominal. As wind speed changes, the control system alters the rotational speed from one assigned value to another based on the pre-determined set of values. The machine then continues to operate in the new rotational speed until another significant variation in wind is observed. Such control actions are necessary and critical for continued operation of wind turbine. However, there are other situations when load variations span for a shorter time and even repeat itself in every cycle of rotation. Such situations could cause sharp peaks in loading, especially 
the cyclic loads result in fatigue loading adversely affecting the lifespan of the turbine. Peaks of aerodynamic loads arising from passing gusts and variations due to interference of tower are valid examples of such situations. Counter acting such short-term variations would require swift alterations to aerodynamics of the rotor that can act within one cycle of rotation.

\subsubsection{Aeroelastic effects of short-term load fluctuations}

Short term variations in wind such as a sudden gust, or rapid aerodynamic changes can result in short-term load fluctuations. The sporadic power fluctuations resulting from such scenarios affect the safe operation of the electro-mechanical devices connected to the turbine, and the electric grid in general. Designing control approaches to counter such swift and short fluctuations in loads is important for reliability in continued power production of turbines. Such approaches would need to target control actions implemented within the period of cyclic rotation, which in nominal operating conditions are in the order of a few seconds. Aerodynamic loads on the rotor are consistent with the wind when the air is free to flow past the rotor. However, presence of the tower creates an obstruction to this flow, which causes a deficit in the available wind potential at the rotor plane when a blade is passing through that region (i.e. in front of the tower on an upwind rotor or behind the tower in a downwind one). This phenomena is observed for every blade and recurs in every cycle of rotation, resulting in cyclic variations in aerodynamic loads on the rotor. The influence of 


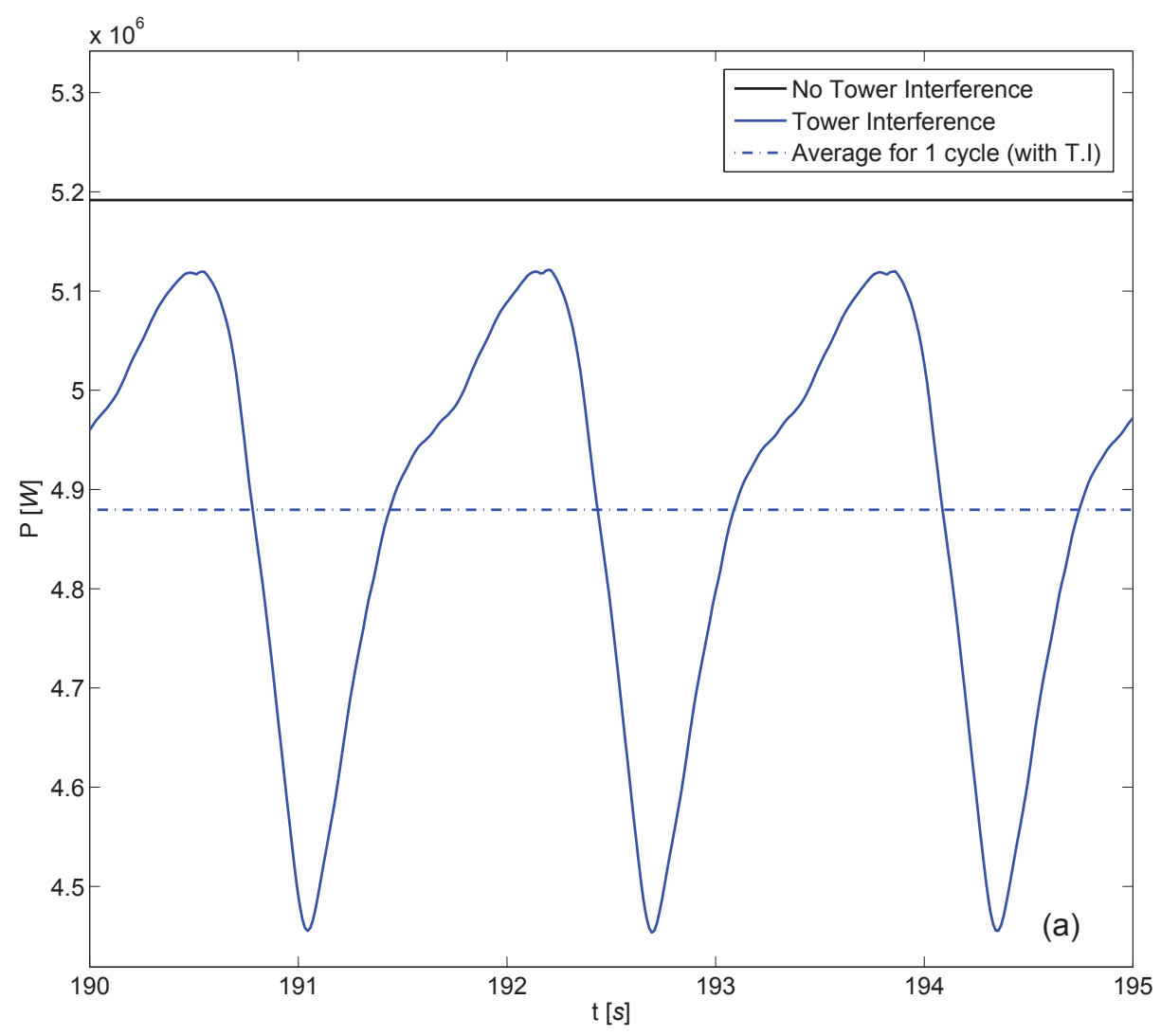

Figure 3.1: Instantaneous power generated $P$ indicative of turbine rotor behavior due to tower interference, plotted against time span of operation that includes one cycle of rotation.

tower on the rotor dynamics has always been of key interest to researchers in wind turbine related studies $[91,92,93]$.

In presence of the tower, certain measurable properties show variations that could impact the performance of the rotors. For example, oscillations in rotor thrust and torque, and power deficits occurring from such cyclical loading conditions have long lasting impact on turbine rotors. The NREL 5MW RWT rotor was subjected to tower interference in nominal operating conditions to understand the dynamic response. Figure 3.1 shows the instantaneous power $P$ during a period of $5 \mathrm{~s}$, which is close to the period of rotation at its nominal 
operational conditions of $12.1 \mathrm{rpm}$. As observed from the figure, the period shows three pulsations in instantaneous power generated that correspond to instances when each blade crosses in front of the tower. Besides adverse effects on the electrical machinery caused by rapid pulsations, there is also a consequential deficit in the averaged power generation that can be noted from the two horizontal lines in figure 3.1. While the solid black line indicates instantaneous power of the same rotor when tower interference effects are neglected, the dotted blue line is an averaged value of instantaneous power in the presence of a tower. This observation of the overall outcome can be primarily attributed to the aerodynamic changes in the rotor, which can be better understood through a closer study of the blade dynamics during these operational regimes.

Wind turbine blades are designed with emphasis to structural stability near the root (use of thicker airfoils) and with increasing aerodynamic efficiency moving away from the root (use of thinner airfoils). This is because the sections of the blade farther from the root $($ span $>50 \%)$ contribute more to the aerodynamic propulsion of the rotor. Lift generated by airfoil sections is highly dependent on the angle of attack $(\alpha)$, and is hence considered the single most important aerodynamic property of turbine blades. The angle of attack $(\alpha)$ at $90 \%$ span section of the rotor blades are studied to determine the cause for variations in power that was observed. Figure 3.2 depicts the angles of attack $(\alpha)$ observed on NREL 5MW RWT rotor blades covering a time period for one cycle of rotation in nominal operating conditions. The sharp fluctuations observed in $\alpha$ corresponds to the time when a blade passes in front of the tower, and reflects the deficit in axial wind due to the 




Figure 3.2: Angle of attack $\alpha$ indicative of rotor blade behavior due to tower interference, plotted against time span of operation that includes one cycle of rotation.

interference. The $\alpha$ behavior observed in a $5 s$ time span shown in figure 3.2 is indicative of the entire blade behavior that will repeat in every cycle of rotation. Another interesting aspect noticeable from the figure is that the interference of tower causing highest variations in $\alpha$ occurs in a time span of about $1 \mathrm{~s}$, and is consistent for every blade. The wide range of fluctuation in $\alpha$ also suggest contrasting aerodynamic loads on the turbine blades occurring in a short span of time. 


\subsubsection{NREL 5MW RWT as a numerical test bed}

The benchmark turbine used in this study is known as the NREL-5MW Reference Wind Turbine (RWT), and their characteristic properties are presented in Table 3.1. Designed with the support from U.S Department of Energy's National Renewable Energy Laboratory (NREL), the intention was to provide a baseline for researchers in the field of wind power. Design features of this machine are based on various state-of-the-art rotors and conceptual designs available at the time of conceptualization in 2009. While various multimegawatt turbines are operational today, the NREL-5MW RWT is still widely used as the baseline in a number of research projects across the globe. An upwind 3-bladed horizontal axis wind turbine with a rated power of $5 M W$, the RWT was designed for use as an offshore turbine. Most relevant to the current study are the flexible blades used on the NREL 5MW RWT rotors, which are $61.5 \mathrm{~m}$ long and weigh up to 18 tons. The blade also function under flexo-torsional modes of operation, which essentially means that they are designed to twist under bending loads to dynamically control the loads during operation. Jonkman et al. [74] provides a comprehensive description of the turbine's design features.

The aeroelastic effects on the rotor due to short-term fluctuations such as tower interference (as discussed earlier) are significant to completely understand the ideology adopted for rapid-load control. The two control methodologies covered in this study are pitching and trailing-edge flap actuation, and will be introduced in the context of rapid control 
Table 3.1

NREL-5MW RWT operational parameters

\begin{tabular}{lr}
\hline Description & Value \\
\hline Rating & $5 \mathrm{MW}$ \\
Rotor Orientation & Upwind \\
Configuration & $3 \mathrm{blades}$ \\
Rotor, hub diameter & $126 \mathrm{~m}, 3 \mathrm{~m}$ \\
Hub Height & $90 \mathrm{~m}$ \\
Rated wind speed & $11.4 \mathrm{~m} / \mathrm{s}$ \\
Rated rotor speed & $12.1 \mathrm{rpm}$ \\
Overhang & $5 \mathrm{~m}$ \\
Rotor pre-cone & $2.5^{\circ}$ \\
\hline
\end{tabular}

scenarios. The aeroelastic analysis of various load-control techniques applied under rapid scenarios are performed on the NREL 5MW RWT under operational conditions defined for the turbine.

\subsection{Rapid pitch control}

Wind turbine blades are complex beam-like structures with airfoil-profile cross sections that vary in their twist angle, sectional shape, and chord length all through the blade span. As a result at every instant of operation, the aerodynamic and structural loads acting across the span are different. On the other hand, mitigating the effects of rapid load variations need predictive counter actions that are fast enough to achieve the desired alterations within a few seconds. Full-span pitching mechanism involves rotation of the entire blade around its longitudinal axis using electric motors or hydraulic actuators located in the rotor hub. 
Though studies on conventional pitching are successful in understanding their ability to mitigate long-term load variations, there is very little knowledge about short-term pitch action. Consequently, the boundaries of full-span pitching as an effective technique for rapid action control are not established.

In context of rapid action, pitching should be swift in countering cyclical variations like the ones caused by tower interference effects and/or the presence of gusts and turbulence. Pitching of blades that cumulatively weigh about $50-55$ tons, while operating under aerodynamic loading and in such short time spans demands immense energy. There is also the potential for aero-elasto-inertial instabilities in the rotor that may compromise the structural integrity or drastically shorten turbine lifespan. This emphasizes the need to study the aeroelastic response of wind turbine rotors for a comprehension of the scope and limits of such rapid pitch-control actions. Pitching as a control action alters the alignment of the blade with the wind at the root, imposing the same angular change along the entire blade. Due to the changes in shape and inflow conditions, the aerodynamic loads acting on the various sections along the span are quantitatively different. However, the changes across the blade span have a similar qualitative pattern, which when integrated along the entire span results in a global response consistent to the control action. Thus, studying the aeroelastic behavior at significant sections could be considered representative of the qualitative dynamics of the entire blade and provides insight into the key physical mechanisms responsible for these dynamics. In any wind-turbine blade, the sections closer to the tip are designed to be more aerodynamically efficient and contribute more to the driving torque, 
which is crucial for power production. These span sections are hence potential regions that provide information on the aeroelastic rotor response to control-pitch action. Selecting the $90 \%$ span section for this analysis promises to show intense vibrational and deformational effects as close as possible to the blade tip without being influenced by the tip effects. This effectively takes into account the combined dynamics of the structural and aerodynamic effects on the blade. The aerodynamic forces acting at each section of the blade directly depend on the angle of attack, $\alpha$, which makes it a key observable for assessing the blade loads. It also reflects the geometrical modifications of the rotor due to dynamic structural deformations and flow characteristics that alter based on the machine kinematics. Hence, in a complex dynamic system like this with coupled aeroelastic modes of operation, an aerodynamic observable such as $\alpha$ serves as a nexus between the geometrical/kinematic aspect of the problem and the dynamic one.

Figure 3.3 shows a schematic of a two-dimensional airfoil blade section, showing the angles relevant to pitch control action, viz. the angle of attack $\alpha$, and its relationship with the angle of pitch $\theta_{p}$, the angle of incidence of the flow $\phi$, and the twist angle of the section $\theta_{t w}$. The twist angle depends on structural properties of the blade such as the design twist angle of sections in the original, un-deformed configuration $\theta_{L b_{z}}$, and the instantaneous deformational twist $\theta_{l L_{z}}$. These properties vary dynamically when pitch control actions are exerted, based on its complex non-linear aeroelastic dynamics. This results in a change in the angle of attack $\alpha$, which does not exactly resemble the change in pitching from the control action, $\theta_{p_{c t r l}}$. As it was mentioned above, $\alpha$ is an essential parameter that defines 


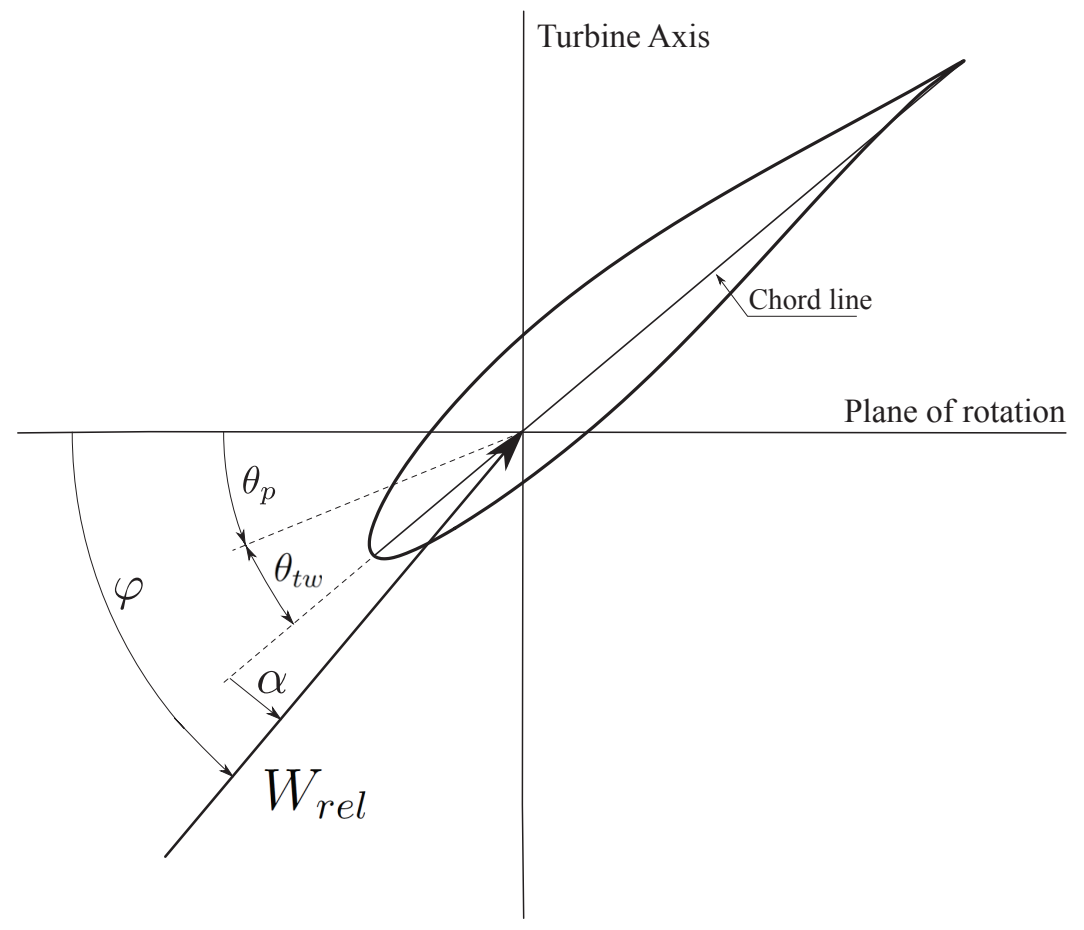

Figure 3.3: Schematic of a 2-D airfoil section of the blade, showing angles relevant to pitch control action.

the aerodynamic forces acting on the section, and thus is the target of the control action itself. Assessing the actual relationship between the control action represented by $\theta_{p_{c t r l}}$ and the corresponding change in $\alpha$ is the first aspect that we shall analyze.

This section of the numerical study explores the extent of rapid pitching and the related aeroelastic rotor response for a benchmark wind turbine for load-control scenarios that are similar to tower interference. A pre-determined control action is used to pitch the blade from one state to the other in a short time-scales and resulting changes in relevant properties of the rotor are studied. The basic test control action is a positive step change in pitch angle, $\theta_{p_{c t r l}}$, applied as a collective pitching of all the blades simultaneously. The simulation of the turbine operation continues for a few more cycles of rotation, as needed for the aeroelastic 
transient modes of the rotor to be captured.

\subsubsection{Aeroelastic blade response to pitching control}

Pitching action as a load-control mechanism during turbine operation works by adjusting the angle of attack $(\alpha)$ on blade sections. Based on the aerodynamic interference of the rotor on the incoming wind, $\alpha$ at each blade section is dependent on the instantaneous orientation the incident flow. The blade designed for use on NREL-5MW RWT is a flexible one [74] and will be referred to as the standard blade. This essentially means that the blade is capable of deforming under operation without causing permanent structural damage. The standard blade also has flexo-torsional modes of operation which allows it to twist under bending loads to optimize blade performance. These factors makes it difficult to attribute the precise cause for changes in $\alpha$ that result from a control action. The blade flexibility obscures the specific contributions towards variation in $\alpha$ from elastic deformations, torsional movement, variation in incident flow, and aerodynamic effects. In order to isolate the direct effect of incident flow alterations due to aerodynamic interference, a hypothetical hyperstiff blade was designed with a structural stiffness $10^{3}$ times that of the standard blade. Without altering the aerodynamic shape of the standard blade or its inertial properties, the higher stiffness ensures negligible deformation. By ruling out deflection and torsion, the hyper-stiff blade allows isolation of aerodynamic interference as the cause for change in $\alpha$ that occurs as a result of certain fixed change in pitching angle $\theta_{p}$. 
At its nominal operation conditions, the NREL-5MW has an initial pitch angle, $\theta_{p_{0}}$ of $0^{\circ}$. The control-action is imposed starting at $20 s$ of stable operation, which is approximately equivalent to 4 cycles of rotation. Figure 3.4 shows the evolution of $\alpha$ for a $90 \%$ span section of the hyper-stiff blade, resulting from a series of prescribed pitch-control actions. A positive pitching (feathering) action results in a drop in the angle of attack from the original value for that section in its nominal operating conditions. It can be observed that this change in angle of attack $\Delta \alpha$ is not equivalent to the control pitch angle $\theta_{p_{c t r l}}$ imposed, but there is a definitive behavior. And as mentioned earlier, this variation in the $\Delta \alpha / \theta_{p_{c t r l}}$ response for the hyper-stiff blade is exclusively related with the changes in aerodynamic interference. Though this observation is significant, a more important study would be on the actual rotor designed for NREL 5MW RWT that uses flexible blades. This rotor is designed to have a pre-cone of $2.5^{\circ}$ and operates with a positive tilt of $5^{\circ}$. A tilted rotor is asymmetric within each cycle of operation, which makes it difficult to isolate oscillations induced by blade movement during pitching. Hence, the test scenarios created for the standard blade also turned tilt off for the rotor. This would be comparable to an actual full-size rotor placed in a virtual wind tunnel with all other dynamics intact. These numerical experiments indicate a consistent dynamic response to rapid pitching similar to that observed for a hyper-stiff blade. Figure ?? shows the evolution of $\alpha$ for a $90 \%$ span section of the standard blade, for the same pitch-control actions. The rates of pitching applied in each case of control pitch angle $\theta_{p_{c t r l}}$ are same for both the blades. Use of the standard blade however, will now result in a different $\alpha$ at the $90 \%$ span section of the blade before the pitching is applied. And the 




Figure 3.4: Time evolution of $\alpha$ at the $90 \%$ span section of hyper-stiff blade, for a series of pitch-control actions of a determined angle $\theta_{p_{c t r l}}$.

variation in $\alpha$ resulting from the rapid pitching also has a consistent behavior. Even though the general behavior is similar to the hyper-stiff blade, the deformational effects introduce new aspects to the dynamic response in the transition period after the control action is complete. These effects and their cause will be discussed in later sections. Nonetheless, it is noticeable that distinct control pitch actions result in a specific alteration to $\alpha$, and these $\Delta \alpha / \theta_{p_{c t r l}}$ response are the result of an aero-elastic response of the blades.

The first aspect to note is the systematic consistency in variation of $\alpha$ achieved when the aeroelastic response stabilizes after a transient period (i.e. the value of the final plateau in 

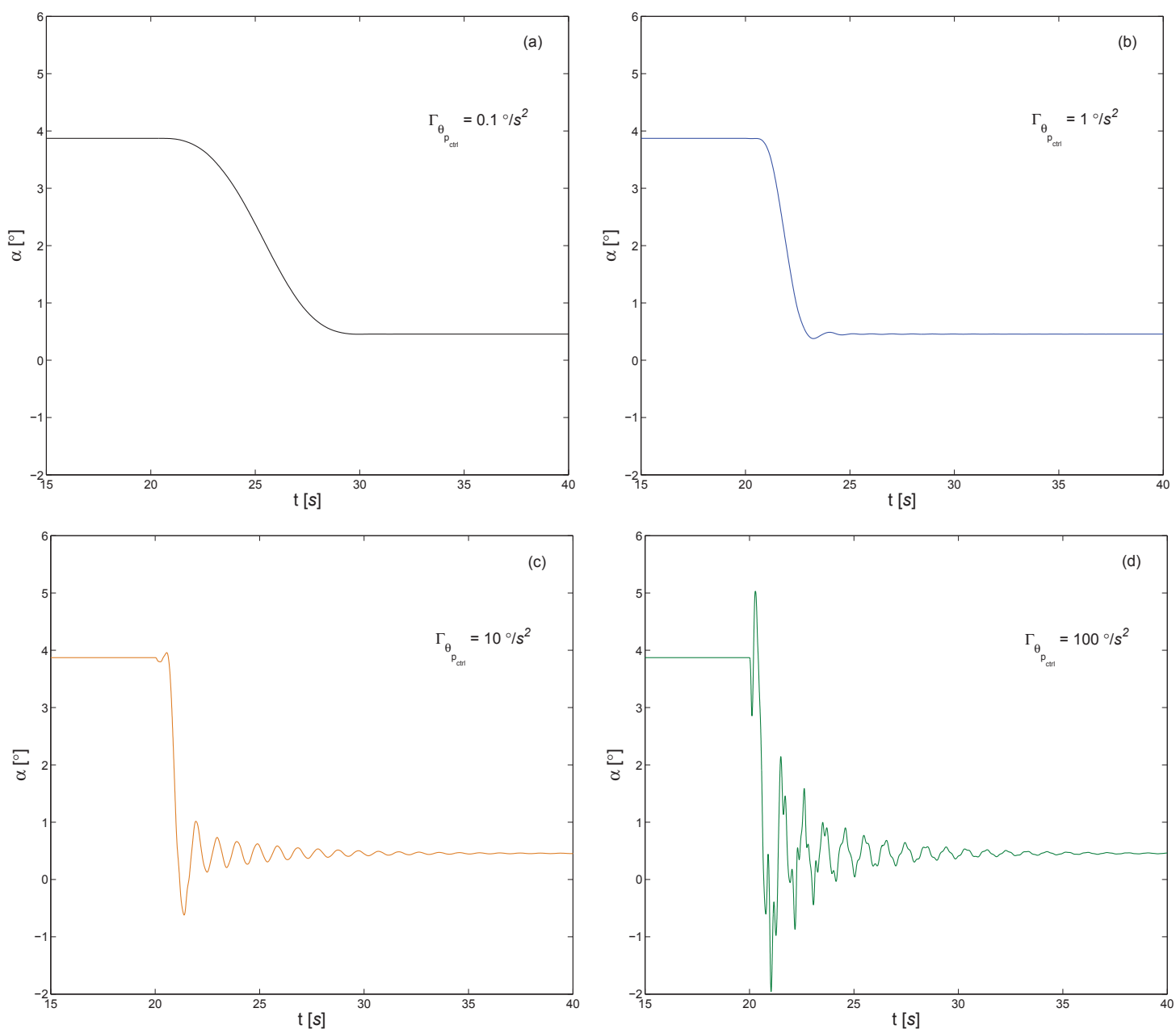

Figure 3.5: Change in angle of attack, $\Delta \alpha$ for a control pitch action of $\theta_{p_{\text {ctrl }}}=5^{\circ}$ with different accelerations of pitching, $\Gamma_{\theta_{p_{c t r l}}}$; (a) $0.1 \% s^{2}$, (b) $1 \% / s^{2}$, (c) $10 \% / s^{2}$, (d) $100 \% s^{2}$.

the different plots), and that this variation depends only on the angle of pitch control imposed, $\theta_{p_{c t r l}}$. This indicates that the end result of the control action is independent of the speed at which pitching takes place, similar to the hyper-stiff blade. However, the transient behavior now depends strongly on the acceleration of pitching. The value of the final plateau in the $\alpha$ signal is different than the one observed in the hyper-stiff blade for a simi$\operatorname{lar} \theta_{p_{c t r l}}$, which is due to the contribution to twist by the torsional deformation. In order to 
verify the behavior observed in the section at $90 \%$ of the blade span, the same analysis was repeated for other sections located along the external (i.e. the aerodynamically active) portion of the blade. As these regions are the major contributors to the aerodynamic functionality, the information presented here is representative of the entire blade. Figure 3.6 shows the $\Delta \alpha / \theta_{p_{c t r l}}$ relation in function of the pitch-control action $\theta_{p_{c t r l}}$ at three different locations along the blade span: $90 \%, 70 \%$, and $50 \%$. The $\Delta \alpha / \theta_{p_{c t r l}}$ relation is shown for both the hyper-stiff blade (dotted-lines) and standard blade (solid lines), and suggests consistent behavior throughout the entire blade. This also serves as a comparison between a purely aerodynamic vs. a fully aeroelastic response. This establishes a non-linearity in aeroelastic response for the entire blade, but indicates a consistent rotor behavior. The $\Delta \alpha / \theta_{p_{c t r l}}$ relation indicates a consistent increase with control pitch angle until about $\theta_{p_{c t r l}}=15^{\circ}$, after which there is a drop. This variation in the aeroelastic response will be discussed later.

The next step in the process is to better understand the dynamic aeroelastic response of the NREL-5MW rotor using the standard blade. The deformational effects and the aeroelastic interaction introduces new aspects in the dynamic response that depend not only on the amount of pitch-control action, but also on how fast this action is applied. An example of this could be seen in figure 3.5, where the change in $\alpha$ is shown for the case of a fixed control action of $5^{\circ}$ of pitch applied during different time-spans, i.e. involving different angular acceleration of the pitching action $\Gamma_{\theta_{p_{c t r l}}}$.

The second characteristic aspect that could be observed in the dynamic response of the 


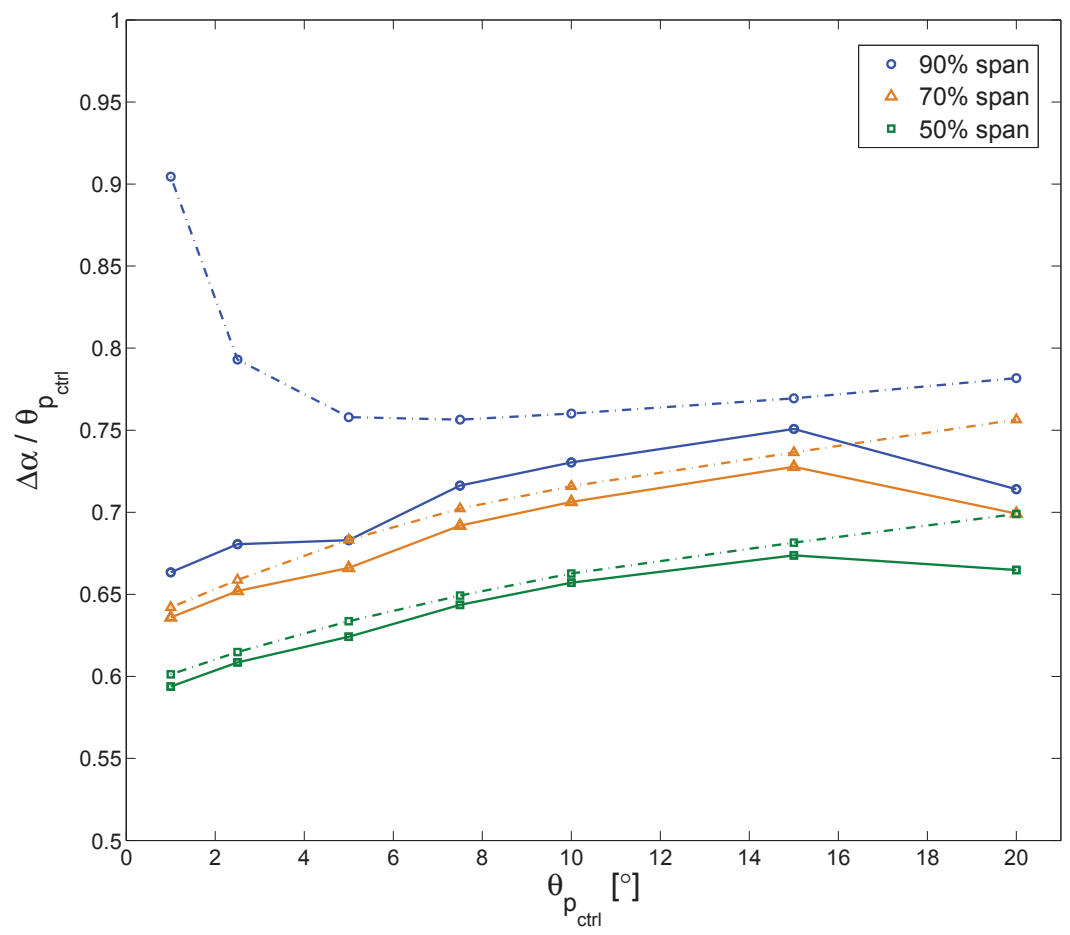

Figure 3.6: The $\Delta \alpha / \theta_{p_{c t r l}}$ relation in function of the pitch-control action $\theta_{p_{c t r l}}$ at different locations along the span. The dotted lines show the curves for the hyper-stiff blade, and the continuous lines the case of the standard blade.
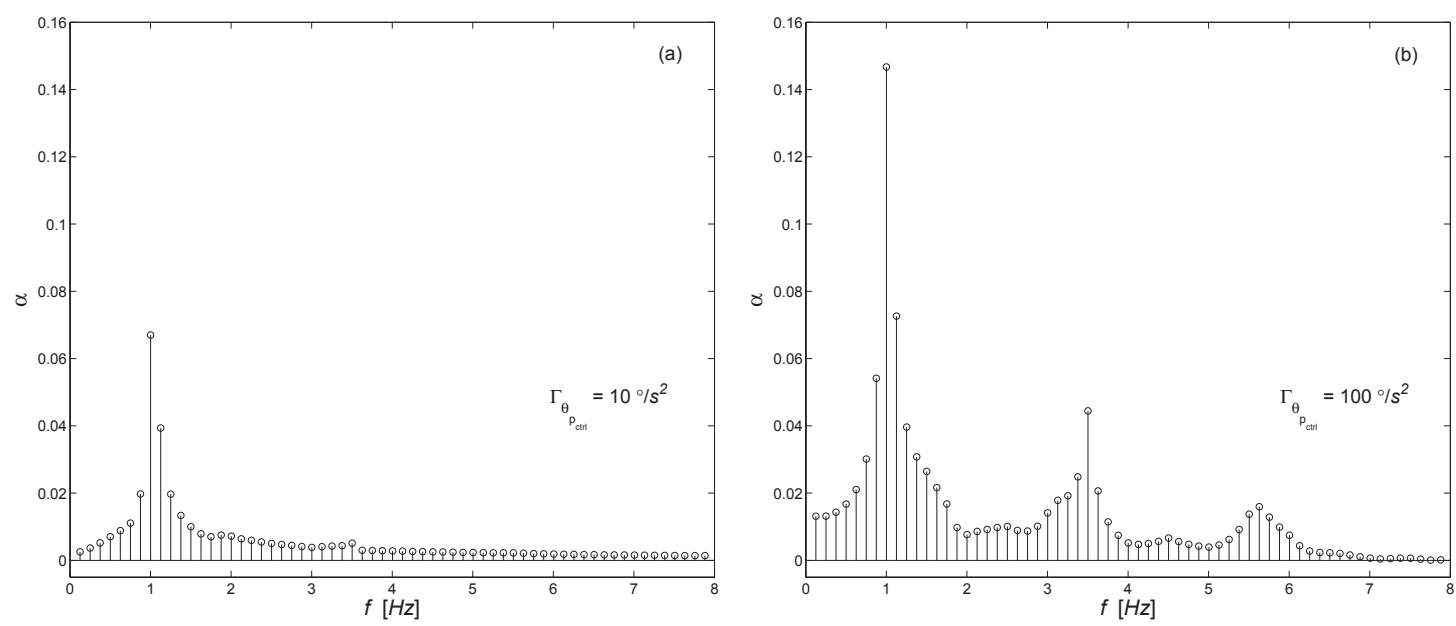

Figure 3.7: Frequency content in the aeroelastic response of the rotor for a pitch actuation of $\theta_{p_{c t r l}}=5^{\circ}$ at two different accelerations of pitching; (a): acceleration of $10^{\circ} / \mathrm{s}^{2}$, (b): acceleration of $100^{\circ} / \mathrm{s}^{2}$. These spectra correspond respectively to cases (c) and (d) in figure 3.5. 
rotor with standard blades, shown in Figure 3.5, is the presence of oscillations in the transient period before a constant value of $\alpha$ is achieved, whose nature depends on the value of pitching acceleration. The four panels in figure 3.5 illustrate four distinctive types of behavior observed at different values of $\Gamma_{\theta_{p_{c t r l}}}$. To illustrate the effect of actuation rate on rapid pitching, a nominal pitch control angle of $\theta_{p_{c t r l}}=5^{\circ}$ is presented. A wide range of acceleration cases were assessed, and the cases shown here covering four orders of magnitude is an overall representation. First, at low values of acceleration such as $\Gamma_{\theta_{p_{c t r l}}}=0.1^{\circ}$ (figure 3.5(a)), the transient evolution of $\alpha$ is completed without oscillations, and is comparable to the behavior observed for hyper-stiff blade. When the level of acceleration is slightly increased to $\Gamma_{\theta_{p_{c t r l}}}=1^{\circ}$ (figure 3.5(b)), a second type of behavior where small noticeable oscillations appear in the aeroelastic response after pitching. This is followed by a range of acceleration values where a consistent behavior of periodic oscillations of a single fundamental frequency, which is depicted by an acceleration value $\Gamma_{\theta_{p_{c t r l}}}=10^{\circ}$ (figure 3.5(c)). Finally, at higher values of acceleration $\Gamma_{\theta_{p_{c t r l}}}=100^{\circ}$ (figure 3.5(d)), the evolution of $\alpha$ has a more complex oscillatory behavior, indicating the presence of richer spectrum of frequencies.

\subsubsection{Oscillatory blade response induced by rapid pitching action}

As shown in the previous section (see figure 3.5), for a given value of the pitch action

$\theta_{p_{c t r l}}$, the evolution in the transient period before the blade reaches a stable configuration 
exhibits four distinctive states in terms of its qualitative behavior, whose nature depends on the value of pitching acceleration. In this section, we focus on the main aspects of the oscillatory response observed at higher pitch-acceleration values, e.g. the states depicted in figures 3.5(c) and 3.5(d). First, we identified the threshold in pitch acceleration that leads to the appearance of single-frequency oscillations and the threshold at which a multifrequency content is observed, i.e. the states whose spectra are depicted in figures 3.7(a) and 3.7(b) respectively. The values of these transitional thresholds depend on the amount of the pitch action $\theta_{p_{c t r l}}$, and are plotted in figure 3.8 for a pitching range from $\theta_{p_{c t r l}}=1^{\circ}$ to $\theta_{p_{c t r l}}=15^{\circ}$. The light-gray shaded region below lower threshold indicates pitching conditions at accelerations low enough to result in an aeroelastic response with minor or negligible oscillations. The shaded region above the upper threshold indicates pitching accelerations at which a multi-frequency content is present. As a way of example, figure 3.8 also includes two markers $(\nabla)$ showing the locus of the cases for $\Gamma_{\theta_{p_{c t r l}}}=10^{\circ} / \mathrm{s}^{2}$ and $\Gamma_{\theta_{p_{c t r l}}}=100^{\circ} / \mathrm{s}^{2}$, which correspond to the spectra shown in figure 3.7, and the evolutions shown in figures 3.5(c) and 3.5(d).

The region in between the two thresholds, shown in white in figure 3.8 , is characterized by a consistent oscillatory behavior with a single fundamental frequency which has a consistent value slightly above $1 \mathrm{~Hz}$, with a slight dependence on the pitch actuation angle, ranging from a minimum of $0.993 \mathrm{~Hz}$ for $\theta_{p_{c t r l}}=1^{\circ}$, to a maximum of $1.071 \mathrm{~Hz}$ for $\theta_{p_{c t r l}}=20^{\circ}$. The oscillations also exhibit a consistent damping that depends only on the value of $\theta_{p_{c t r l}}$, examples of which can be observed in the time evolutions of $\alpha$ shown in figure 3.9 for four 


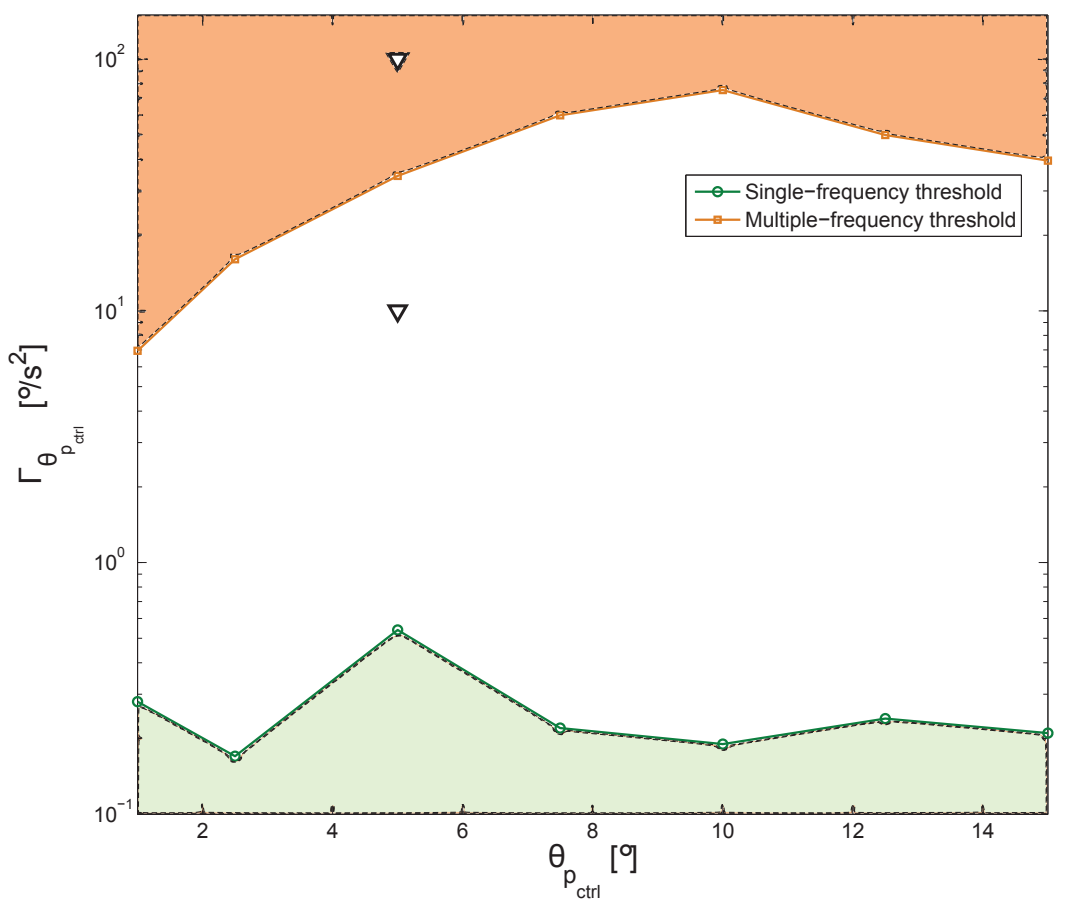

Figure 3.8: Thresholds on acceleration of pitching actuation for the appearance of singlefrequency periodic oscillations, and for the appearance of multiple-frequency content. Markers $(\nabla)$ indicate the locus of cases for $\Gamma_{\theta_{p_{c t r l}}}=10^{\circ} / \mathrm{s}^{2}$, and $100^{\circ} / \mathrm{s}^{2}$, at $\theta_{p_{c t r l}}=5^{\circ}$, corresponding to the examples shown in figures $3.5(\mathrm{c})$ and $3.5(\mathrm{~d})$, and the frequency spectra shown in figure 3.7 .

different pitching angles from $\theta_{p_{c t r l}}=1^{\circ}$ to $\theta_{p_{c t r l}}=20^{\circ}$. This exclusive dependency on an aerodynamic variable such as $\theta_{p_{c t r l}}$ indicates that the damping is predominantly aeroelastic in nature, more than purely due to material properties of the blade structure. The four different cases plotted in figure 3.9 illustrate the change in damping as $\theta_{p_{c t r l}}$ increases, going progressively from rapid attenuation to actual amplification of the aeroelastic vibrations, with a zero damping situation (where the amplitude remains practically constant) occurring at about $\theta_{p_{c t r l}}=16.25^{\circ}$. Figure 3.10 shows the value of the logarithmic decrement $\delta$, characterizing the damping at the nominal wind speed of $11.4 \mathrm{~m} / \mathrm{s}$ for different values of $\theta_{p_{c t r l}}$, and the solid line connecting the markers represents the best-fit cubic curve to 


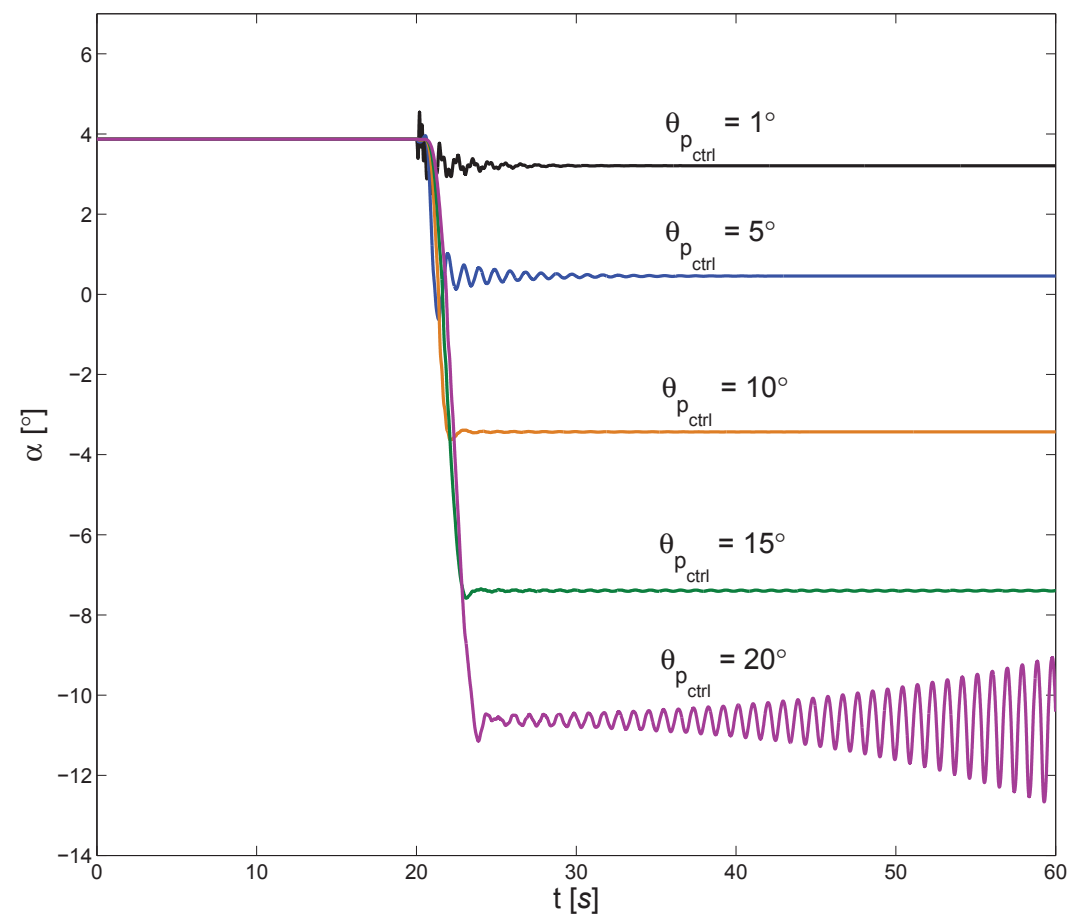

Figure 3.9: Examples of time evolution of the angle of attack $\alpha$ for five different pitching angles, $\theta_{p_{c t r l}}$.

the data. This qualitative behavior of the oscillations, in terms of frequency and damping observed at nominal wind speed is also consistently exhibited at higher values of the operational wind speed, which will be the focus of sub-section 3.2.3.

Verification studies conducted on global rotor parameters during the rapid pitch actuation shows consistency with previous observations about blade-section parameters. The output power P, rotor thrust T, and blade-tip displacement, $U_{h_{x_{t i p}}}$, were assessed in similar test conditions for rapid pitching. A positive pitching (feathering) produced drop in output power, and the final value showed dependency only on the amount of pitch actuation, $\theta_{p_{c t r l}}$. This behavior is qualitatively identical to the one observed in the time evolution of $\alpha$ for the airfoil section at $90 \%$ of the blade span (see figure 3.5), which support our initial selection 


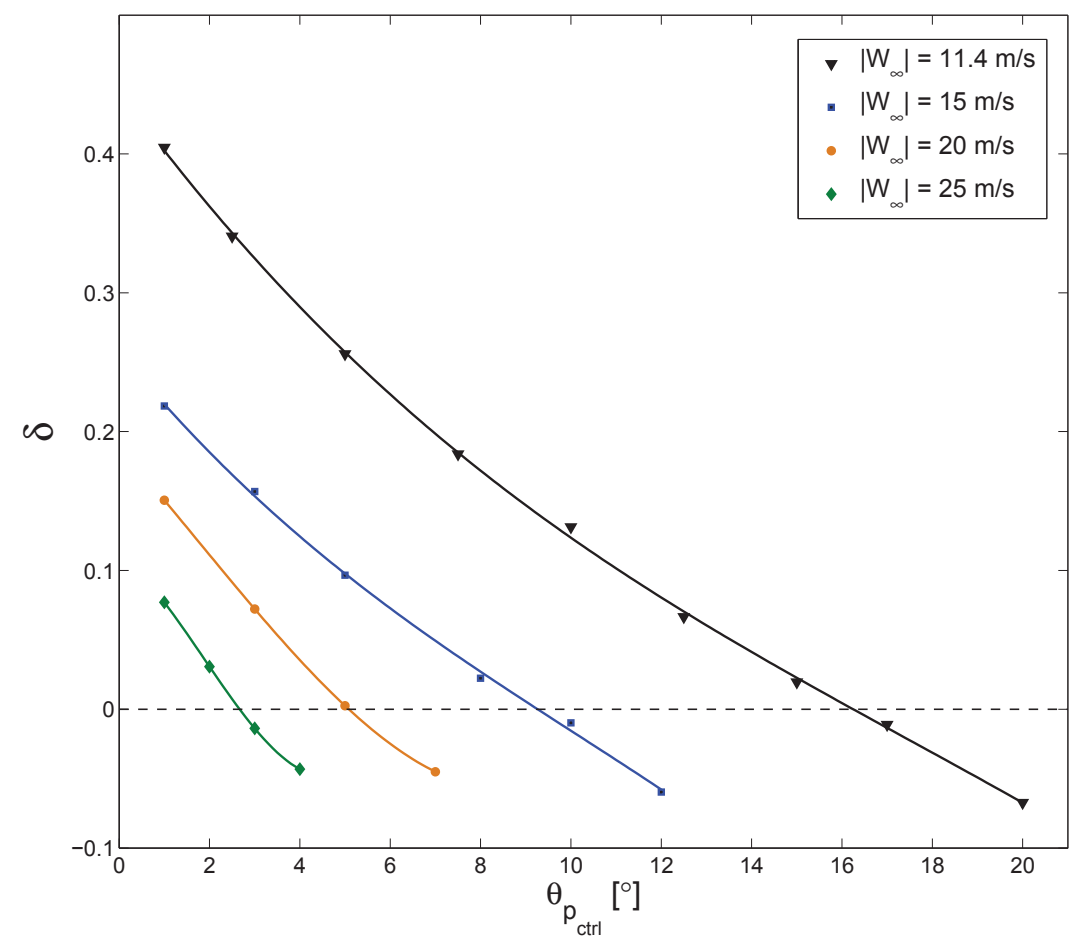

Figure 3.10: Logarithmic decrement $\delta$, characterizing the damping at the nominal wind speed of $11.4 \mathrm{~m} / \mathrm{s}$ for different values of $\theta_{p_{c t r l}}$ (the solid line connecting the markers represents the best-fit cubic curve to the data). Also shown is the damping behavior for three wind speeds above nominal, $W_{\infty}=15,20$, and $25 \mathrm{~m} / \mathrm{s}$.

of $\alpha$ at this location as a significant parameter to study. Accordingly, blade-tip displacement and rotor thrust also show similar behavior on their time evolution.

\subsubsection{Rotor response to rapid pitching for wind speeds above the nom-}

\section{inal}

Figure 3.10 also shows the results for $\delta$ at three different wind speeds above the nominal:

$15 \mathrm{~m} / \mathrm{s}, 20 \mathrm{~m} / \mathrm{s}$, and $25 \mathrm{~m} / \mathrm{s}$, which covers the entire upper range of operation for the NREL-5MW RWT until its cut-off wind speed. The objective of this part of our study was 
to assess the consistency in rotor response to rapid control actions for operational conditions at which the initial pitch $\theta_{p_{0}}$ is no longer zero as it was in the case of the nominal speed. That is, the rapid control action adds to an initial existing pitch which is required to maintain the output power at its rated level for wind speeds above nominal. For the examples shown in figure 3.10, the rotor blades operate at a pitch angle of $\theta_{p_{0}}=9.27^{\circ}$ for wind speed of $15 \mathrm{~m} / \mathrm{s}, \theta_{p_{0}}=16.2^{\circ}$ for $20 \mathrm{~m} / \mathrm{s}$, and $\theta_{p_{0}}=21.8^{\circ}$ for $25 \mathrm{~m} / \mathrm{s}$ (see Table 2 from Ponta et al. $[70])$.

The behavior observed at these higher wind conditions is qualitatively equivalent to the nominal case. That is, final values of $\alpha$ only depend on the corresponding angles of pitch actuation, and the characteristics of the oscillatory motion during the transient period until a stable value of $\alpha$ is achieved depends on the pitching acceleration. The damping behavior is also consistent, with a dependence only on the pitch actuation angle. In figure 3.10 we could see that the value of $\delta$ for the same $\theta_{p_{c t r l}}$ decreases as wind speed increases. This is a consequence of the change in geometrical configuration of the rotor, which is subjected to a higher deformation when operating at higher wind speeds. Angle of the rapid pitching action at which a zero-damping situation occurs $\left(\theta_{p_{\operatorname{ctrl}(\delta=0)}}\right)$ also reduces as the wind speed increases. This is expected due to the fact that the pitching action, $\theta_{p_{c t r l}}$, starts not from zero as in the nominal case, but from a required initial pitch $\left(\theta_{p_{0}}\right)$ that increases with wind speeds beyond the nominal value. However, the total value of pitching angle at which zerodamping occurs $\left(\theta_{p_{(\delta=0)}}=\theta_{p_{0}}+\theta_{p_{c t r l(\delta=0)}}\right)$ increases. Figure 3.11 shows the curves for both $\theta_{p_{\operatorname{ctrl}(\delta=0)}}$ and $\theta_{p_{(\delta=0)}}$ vs wind speed, markers correspond to the same four wind speeds cases 




Figure 3.11: $\theta_{p_{c t r l(\delta=0)}}$ and $\theta_{p_{(\delta=0)}}$ vs wind speed. Markers correspond to the same four wind speed cases shown in figure 3.10, and solid lines show their respective best-fit curves based on a cubic polynomial fitting.

shown in figure 3.10, and solid lines show their respective best-fit curves based on a cubic polynomial fitting.

The fundamental frequencies for these oscillations in cases of wind speeds above nominal also show a consistent behavior with the value slightly above $1 \mathrm{~Hz}$, with a slight dependence on the pitch actuation angle, $\theta_{p_{c t r l}}$. For wind speed of $15 \mathrm{~m} / \mathrm{s}$, the value of frequency ranges from a minimum of $1.012 \mathrm{~Hz}$ for $\theta_{p_{c t r l}}=1^{\circ}$ to a maximum of $1.06 \mathrm{~Hz}$ for $\theta_{p_{c t r l}}=12^{\circ}$. For wind speed of $20 \mathrm{~m} / \mathrm{s}$, the value of frequency ranges from a minimum of $1.031 \mathrm{~Hz}$ for $\theta_{p_{c t r l}}=1^{\circ}$ to a maximum of $1.063 \mathrm{~Hz}$ for $\theta_{p_{c t r l}}=7^{\circ}$. For wind speed of $25 \mathrm{~m} / \mathrm{s}$, the value of frequency ranges from a minimum of $1.045 \mathrm{~Hz}$ for $\theta_{p_{c t r l}}=1^{\circ}$ to a 
maximum of $1.063 \mathrm{~Hz}$ for $\theta_{p_{c t r l}}=4^{\circ}$.

\subsection{Rapid actuation of trailing-edge flaps}

Trailing-edge flaps as active flow-control devices can be used in dynamically controlling the aerodynamic loads on wind turbine rotors through alteration of aerodynamic characteristics of the blades during operation. These devices can manipulate the airflow dynamics near the region of attachment resulting in an increase or decrease in the lift behavior. As fractional chord devices used on short span lengths of the blade, they are lighter and hence easier to be actuated rapidly. Such devices have a great potential in mitigating loads variations that occur in short time spans, such as that caused by tower interference. The assessment of trailing-edge flaps as rapid load-control devices discussed in this section is a represents the wider usage of flow-control devices in active control of dynamic loads for short time-scale fluctuations.

The current study focuses on understanding the rotor response with the use of a $20 \%$-chord Clark Y profile flap as an active trailing-edge device attached on the NREL 5MW RWT rotor blades. The flap as depicted in figure 1.4 spans for $6.15 \mathrm{~m}$ (i.e. $10 \%$ span of the blade) and is attached on all three blades. This covers about $75 \%$ of the aerodynamically active region of the blade. Two dimensional behavior of two key airfoils, NACA $64_{3}-618$ and DU 93-W-210, when attached with a Clark Y flap are available for the study, as mentioned 
in section 2.2 in chapter 2 . In the current study, span regions of the RWT rotor blades that are attached with the Clark Y flaps contain NACA $64_{3}-618$ airfoils. The aerodynamic data characterizing Clark Y flap behavior are available for a range of actuation angles that makes pragmatic sense to their use as dynamic control devices. This is defined by the relative angle between the airfoil and flap denoted by $\beta$, and ranges from $-5^{\circ}$ to $5^{\circ}$. The assessment of trailing-edge flaps control devices for rapid action for short-term load variations are based on pre-determined time spans based on the observations about tower interference, made in section 3.1.1. The control approach actuates the flaps on all three blades from one static relative position to another, and the rotor response during this period is evaluated. The change in relative angle $\beta$ from one configuration to the other is considered as the control action and is known as the control flap-actuation angle, $\beta_{c t r l}$. The primary test scenario is a negative step change in flap control angle, $\beta_{c t r l}$, which changes the relative position of the flap from one static angle to another. The control actuation is applied on all blades simultaneously, and is designed to be completed within one rotation cycle. The simulation of the turbine operation continues for a few more cycles of rotation, as needed for the aeroelastic transient modes of the rotor to develop into a stable state of operation.

The primary interest in most dynamic load control approaches are to curtail the power production and decrease the deformation causing aerodynamic loads on the rotor. Using trailing-edge flaps in rapid control situations proves to be an effective approach in this respect. As discussed earlier, the airfoil-flap configuration had an upper limit of $\beta=$ 
$-5^{\circ}$ and a lower limit of $\beta=5^{\circ}$. This allowed the assessment of a wide range of flapactuation control scenarios by actuating the flap from a configuration of $\beta=0^{\circ}$ to distinct configurations defined by the flap-actuation control angles, $\beta_{c t r l}=-5^{\circ},-2^{\circ},-1^{\circ}, 1^{\circ}, 2^{\circ}$, and $5^{\circ}$.

\subsubsection{Rapid actuation of flap as dynamic load control}

Analysis of the aeroelastic response of turbine rotors in trailing-edge flap configurations indicates that blade-spans with the flap have slightly different behavior compared to regions (without a flap) that are in their original configuration. This variation in response primarily inferred through the angle of attack $(\alpha)$ showed a dependency on the angle of flap-actuation $(\beta)$ as well. Since the aerodynamics of the rotor is now dependent on the flap configurations, the aeroelastic response to control actions cannot be entirely understood by the observation of $\alpha$ alone. They are now the result of combined effects from the instantaneous $\beta$ and the resulting $\alpha$ observed at the blade section. Hence, the effects of such rapid control action are evaluated from a global perspective by evaluating four key aspects of the rotor response. The most key among these are the structural impact due to axial loading and effects on power production. At the turbine, these effects are assessed based on the axial rotor thrust, $T$, and the instantaneous rotor power, $P$. The structural deformations on the blades are also studied from the changes in tip displacement, which is 
observed at the $h u b$ in the axial direction and denoted as $U_{h_{x} \text { tip }}$. And the aerodynamic response at the rotor level is assessed from the changes in $\alpha$ observed at the $90 \%$ span section of the blade, which typically represents the entire blade behavior. Figure 3.12 presents the representative behavior of these four properties for two rapid-control cases where the flap is actuated for $\beta_{c t r l}=-5^{\circ}$ (depicted by the blue curves) and for $\beta_{c t r l}=5^{\circ}$ (depicted by the green curves). They are an overall representation of the dynamic rotor response to rapid flap-actuation when the respective control actions are implemented in a $1 s$ time period.

The scenarios presented in figure 3.12 show the outer limits for the range of flap actuation covered in this rapid-control study. And these properties illustrate the outer boundaries of effective alterations possible in the airfoil-flap configurations under consideration. In the case of $\beta_{c t r l}=-5^{\circ}$, figure 3.12(a) shows a reduction in axial thrust on the rotor easing the aerodynamic loads acting on the rotor as the result of the control action. This reduction of about $17 k N$ is relevant for sudden fluctuations in wind, improving the reliability and lifespan of turbines through better management of fatigue loads. One of the most important effect is an overall power reduction as seen in figure 3.12(b), which is attained through a drop in lift created by the dissipation of flow energy. It shows a reduction of about $76 \mathrm{~kW}$ in generated power by actuating the flap in a nose-down direction of $\beta_{c t r l}=-5^{\circ}$. The ability to effect such power reduction, demonstrated by a light-weight fractional-chord device through a small angle of control actuation is significant in developing innovative control strategies. The rotor response illustrated by tip deflection $U_{h_{x_{t i p}}}$ in figure 3.12(c) shows a reduction in the bending deformation of the blade. This is consistent with the reduced axial 

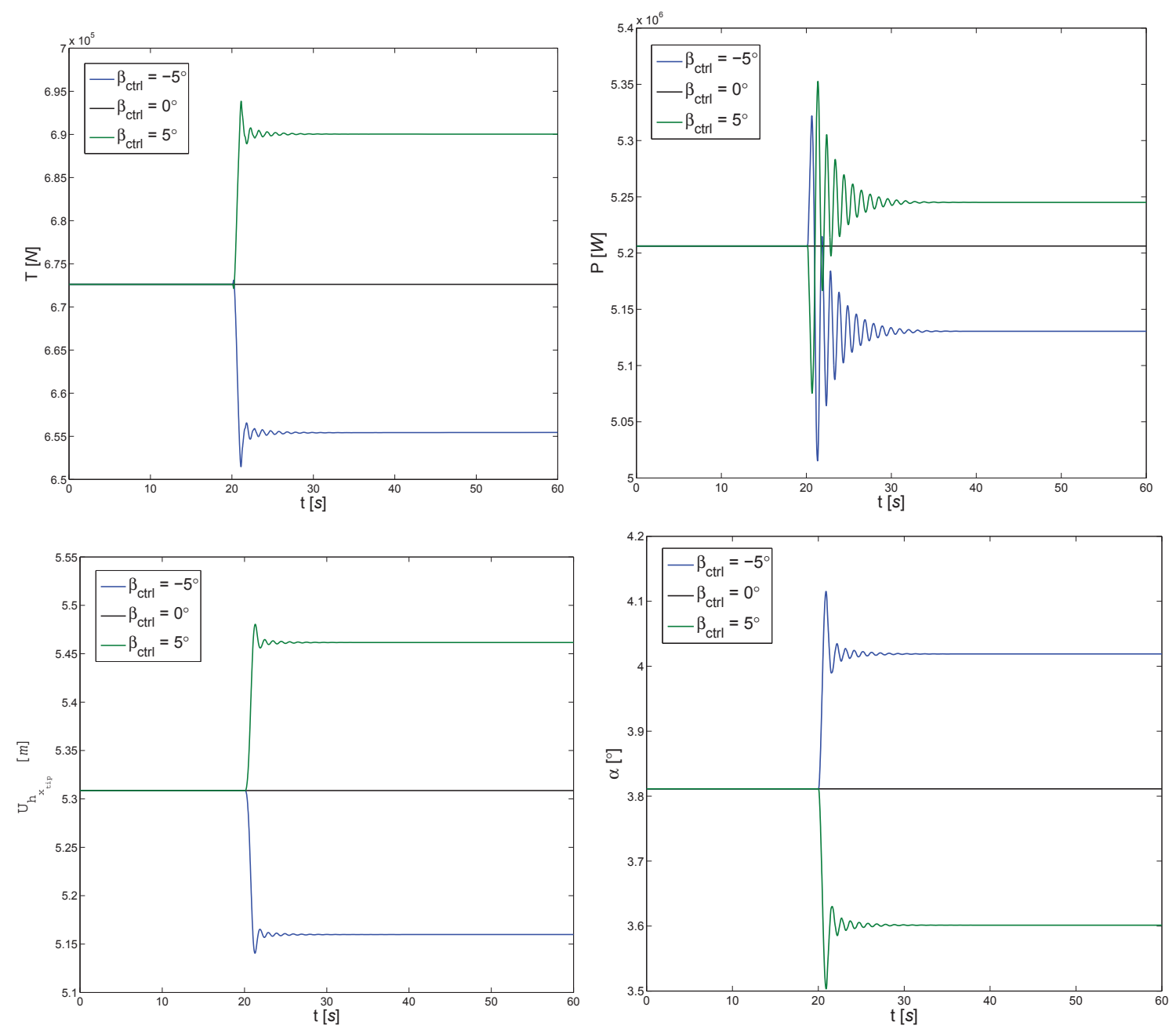

Figure 3.12: Aeroelastic response of the turbine rotor to rapid control action for flap actuation $\beta_{c t r l}=-5^{\circ}$, and $5^{\circ}$ implemented in time-span of $1 \mathrm{~s}$; panels are presented to show the aerodynamic observable, deformational response, and global effects in terms of force and power. Panels (a) rotor thrust $T$, (b) instantaneous power $P$, (c) tip deflection $U_{h_{x_{t i p}}}$, and (d) angle of attack $\alpha$ at $90 \%$ span section. The case for $\beta_{c t r l}=0^{\circ}$ is shown as reference when a flap is attached but not actuated.

loading observed on the rotor as the result of the control action. It is also noticeable from figure 3.12(d) that actuating the flap in the nose-down direction effects a slight increase in the angle of attack $(\alpha)$ observed at the $90 \%$ span section of the blade. Though the change in $\alpha$ observed across the span could vary, the response depicted here at the $90 \%$ represents the overall aerodynamic behavior of the blade and hence the rotor. In understanding the overall 
rotor response, figure 3.12(d) suggests that the amplitude of oscillations in instantaneous power is relatively higher compared to other properties presented. This is expected in a rotor that operates under various modes of vibrations, but the more important observation here is that these oscillations are quickly damped and it attains a stable value of power in the new configuration. The final values attain by these properties have a consistent characteristic that it is purely dependent on the flap-actuation control angle $\beta_{c t r l}$ and does not vary with the rate of flap actuation.

This observation is consistent with all the flap-actuation control angles studied here, i.e. $\beta_{c t r l}=-5^{\circ},-2^{\circ},-1^{\circ}, 0^{\circ}, 1^{\circ}, 2^{\circ}$, and $5^{\circ}$. Figure 3.13 shows the final values observed for the same four properties at the end (or as a result) of the control action, plotted for each flapactuation control angle evaluated. The final values are indicated by the respective markers in each panel of the figure, and the solid lines are the best fit curve illustrating the trend. Beginning at the neutral zero-actuation scenario, there is a steady drop in rotor thrust $T$ with increasing negative angles of control actuation $\left(\beta_{c t r l}<0\right)$, and a similar increase with positive actuation $\left(\beta_{c t r l}>0\right)$. This presents a linear pattern in rotor thrust $T$ as a direct response to the control action, as depicted in figure 3.13(a). An interesting observation is made about the effect on instantaneous power $P$, which shows a cubical dependence on the flap-actuation control angle $\beta_{c t r l}$, as presented in figure 3.13(b). The proportional increase in power at higher angles of actuation is consistent with more driving torque from the higher lift generation associated with positive increase in $\beta$. Correspondingly, there is a linear increase in the tip deflection $U_{h_{x t i p}}$ observed and the computed rotor thrust $T$, which 

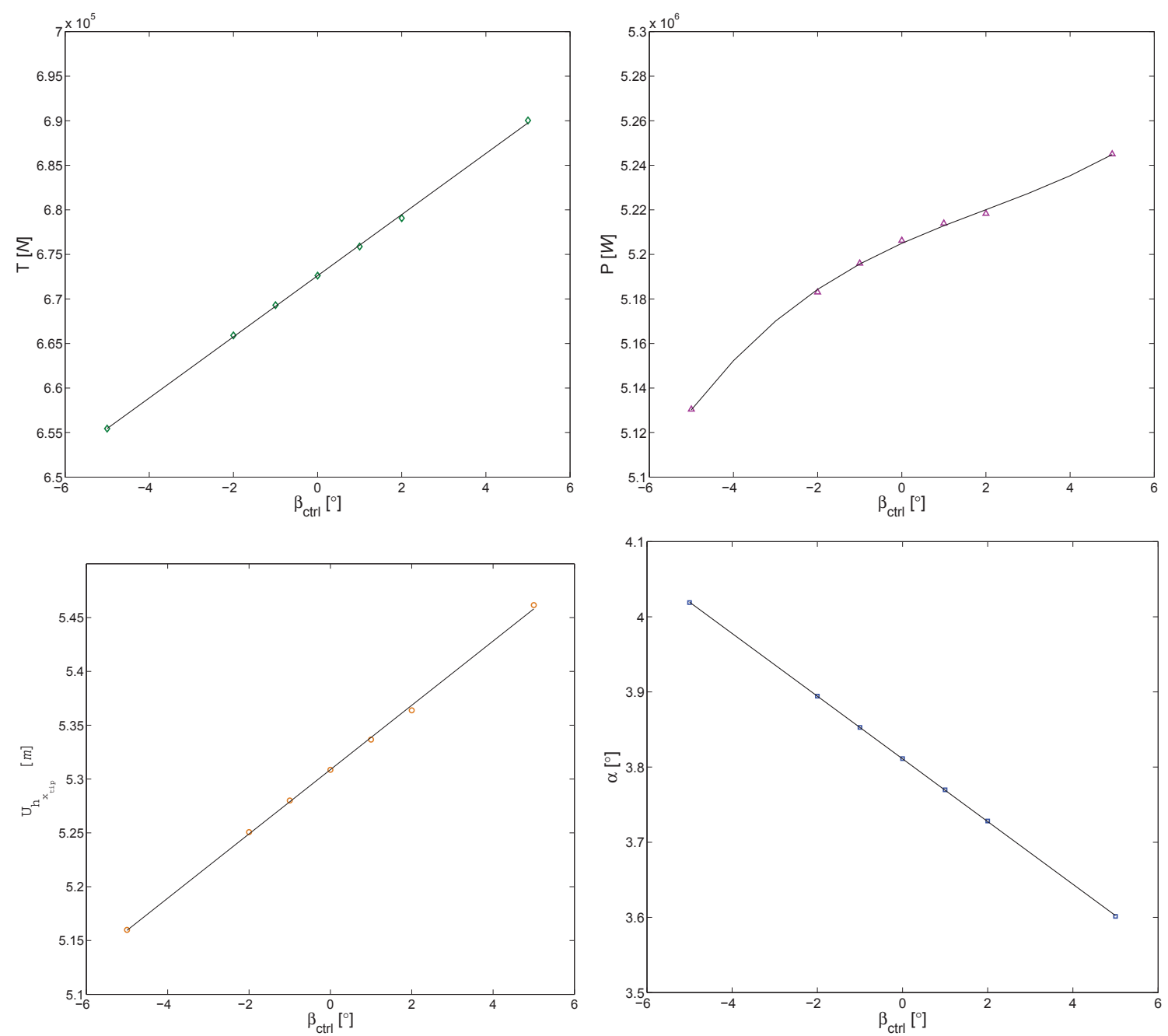

Figure 3.13: Consistent variation in rotor behavior for angles of flap actuation ranging between $\beta_{c t r l}=-5^{\circ}$ and $+5^{\circ}$, showing an aerodynamic observable, deformational response, and global effects in terms of force and power. Panels show the final value of (a) rotor thrust $T$, (b) instantaneous power $P$, (c) tip deflection $U_{h_{x_{t i p}}}$, and (d) angle of attack $\alpha$ at $90 \%$ span section, plotted against angle of rapid flap-actuation control $\beta_{c t r l}$.

can be seen in figure 3.13(b) and (c). It can be observed from figure 3.13(d) the aerodynamic response of the blade decreases linearly with and increasing angle of flap-actuation control. Observations about these trends in tangible properties shown in figure 3.13 are also consistent with the limits of flap-actuation presented in respective panels of figure 3.12. 



Figure 3.14: Oscillatory behavior in rotor response observed for a case of $\beta_{c t r l}=-5^{\circ}$ that effects a reduction in rotor thrust $T$, at increasing rate of flap actuation - (a) $\Delta t_{c t r l}=$ $2 \mathrm{~s}$, (b) $\Delta t_{c t r l}=1 \mathrm{~s}$ (c) $\Delta t_{c t r l}=0.2 \mathrm{~s}$, and (d) $\Delta t_{c t r l}=0.1 \mathrm{~s}$.

The oscillatory behavior of the turbine blades and their effect on the rotor was also evaluated by studying the dynamic response at four different time spans of control action, $\Delta t_{c t r l}$. Keeping $\Delta t_{c t r l}=1 \mathrm{~s}$ as the reference for rapid variations in dynamic loads observed, three other time spans were adopted. Figure 3.14 shows the rotor thrust $T$ behavior during the rapid flap-actuation control when the control is implemented at four different time spans of control actuation. The corresponding rates of flap-actuation are also indicated as an inset 
in the respective panels. The reference case of $\Delta t_{c t r l}=1 s$ resulted in a variation in $T$ as shown in figure $3.14(\mathrm{~b})$, which shows the presence of minor oscillations, noticeable as the effect of the control action that swiftly disappear. A slightly slower scenario was assessed with $\Delta t_{c t r l}=2 s$, which is still within one cycle of rotation of the rotor. The effect of the control action on $T$ is shown in figure 3.14(a), where the resulting $T$ value is immediately obtained and any oscillations present in this scenario are negligible. On the other hand, with increasing rates of flap actuation the oscillatory behavior becomes more noticeable with secondary and possibly tertiary frequencies that contribute significantly to the rotor response. These effect are reflected through increasing amplitudes of oscillations shown in figures 3.14(c) and (d). The final value of $T$ is attained after noticeable oscillations showing rich spectral behavior of the various modes of vibrations resulting from the rapid control action. The most interesting aspect here are the higher rates of damping (relative to those observed in rapid-pitching) involved, which ensures the system stabilizes in its new configuration within a short period of time.

\subsubsection{Power consumption in rapid control}

Considering the short time spans in which these control actions are implemented, a more important aspect is an assessment of power required for actuation, and placing it in relation to the effective alteration in generated power. This section evaluates instantaneous power generated $P$ and the instantaneous power required for control action $P_{c t r l}$ as a baseline 
reference for the comparative analysis of rapid-pitch control and rapid flap-actuation control. First, we will study the instantaneous power $P$, which is the total power output from the turbine computed at each instant of time during operation. As the primary interest in load control is curtailment of power generation, the scenarios of control actuation primarily assessed will be for power reduction. Qualitatively, a positive pitching (feathering) and negative flap-actuation (nose-down) effect a reduction in power. While in both the case the control device (blade or flap) is actuated in a nose-down orientation, due the conventions the pitching action is considered positive and flap-actuation as negative. Quantitatively however as trailing-edge flaps are spread across smaller span sections of the blade, they are intended to produce lesser overall power reduction in comparison to conventional pitching. On the other side, being lighter devices compared to the entire blade (in pitching), flap-actuation is expected to employ lesser power for the control action itself.

As the total alterations in instantaneous power through flap-actuation are lesser, the extreme case for power reduction was chosen and a corresponding effect through rapid-pitching was matched. Based on this idea it was determined that a power reduction effected by a nosedown $\beta_{c t r l}=-5^{\circ}$ can be matched by a feathering action of pitching with $\theta_{p_{c t r l}}=0.35^{\circ}$. In both cases, a reduction of about $76 \mathrm{~kW}$ is obtained irrespective of the rate of control action. Figure 3.15 shows the evolution of generated power $P$ during the rapid control actions, plotted against the time of turbine operation covering a span where the control action is completed. They are plotted top-down in increasing rapidity of control action, and comparing the effects from rapid-pitching presented on the left side to corresponding rapid 

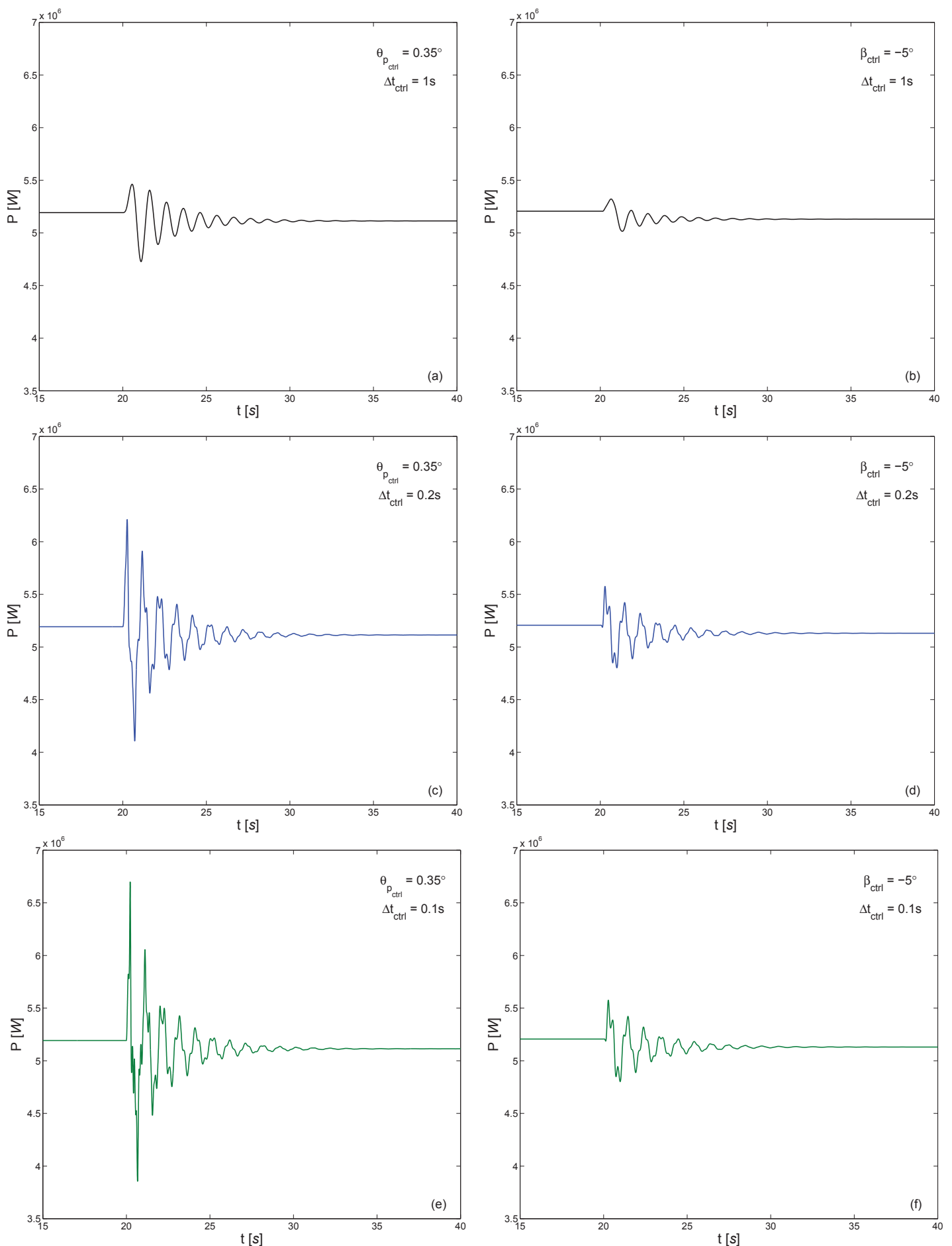

Figure 3.15: Evolution of instantaneous power $P$ during nominal operating conditions due to rapid control action resulting in power reduction, presented for $\theta_{p_{c t r l}}=0.35^{\circ}$ on the left compared to $\beta_{c t r l}=-5^{\circ}$ cases on the right. Panels (a) and (b) show cases with $\Delta t_{c t r l}=1 \mathrm{~s}$, (c) and (d) $\Delta t_{c t r l}=0.2 \mathrm{~s}$, and (e) and (f) $\Delta t_{c t r l}=0.1 \mathrm{~s}$. 
flap-actuation depicted on the right side. That is, control action time span, $\Delta t_{c t r l}$ is the same for panels (a) \& (b), (c) \& (d), and (e) \& (f). Observing the plots top-down, it can be observed that with growing rapidity of control action, the increase in amplitude of oscillations are significantly huge for rapid pitch-control in comparison to rapid flap-actuation control. Both approaches introduce secondary modes of oscillation at higher rates of actuation, and this is attributed to the structural response of the rotor in this context. These oscillations are damped through the course of turbine operation after the control action is completed, but their presence is significant in understanding the effects on mechanical and electrical components associated with the turbine operation. These oscillations are inevitable in any rapid control action, and in this context the flap-actuation control is more favorable as at higher rapidity of actuation, the growth in oscillation amplitudes is minimal.

In a similar comparative assessment of rapid-pitch and rapid flap-actuation controls, scenarios were evaluated for an effective increase in generated power as the result of the control action. This increase will occur from a negative pitching (pitching to stall) or a positive flap actuation (nose-up). The limit in the opposite direction for flap-actuation would be given by a control action of $\beta_{c t r l}=5^{\circ}$ that will result in a proportional increase in instantaneous power generated $P$. However, due to the non-linear behavior in generated power against rapid flap-actuation angle, the increase in power from $\beta_{c t r l}=5^{\circ}$ is about $39 \mathrm{~kW}$. This variation in power is matched by a pitching action of $\theta_{p_{c t r l}}=-0.2^{\circ}$. Figure 3.16 shows the evolution of instantaneous power generated $P$ during the rapid control actions, plotted against the time of turbine operation covering a span where the control action is completed. 

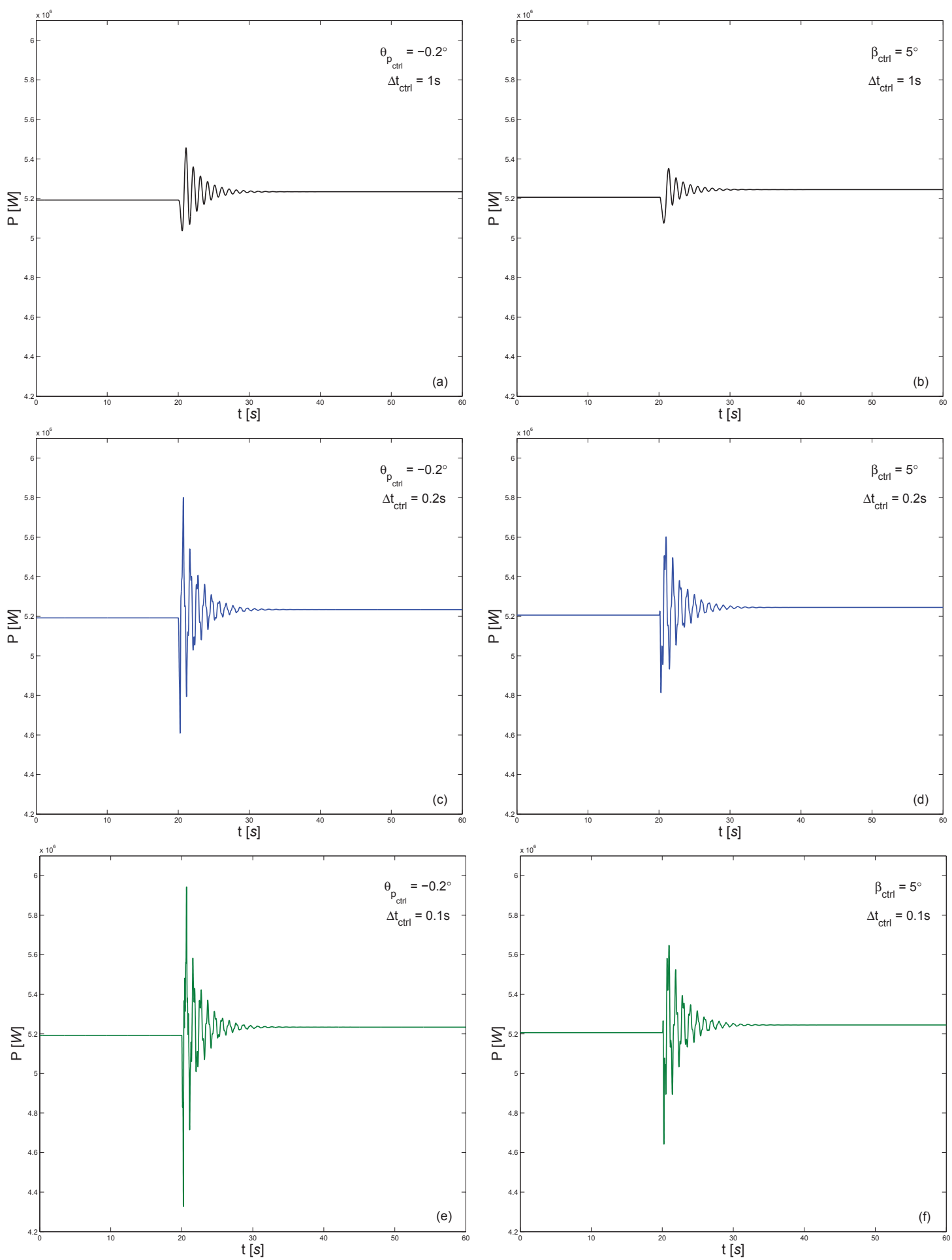

Figure 3.16: Evolution of instantaneous power $P$ during nominal operating conditions due to rapid control resulting in power augmentation, presented for $\theta_{p_{c t r l}}=-0.2^{\circ}$ on the left compared to $\beta_{c t r l}=5^{\circ}$ cases on the right. Panels (a) and (b) show cases with $\Delta t_{c t r l}=1 \mathrm{~s}$, (c) and (d) $\Delta t_{c t r l}=0.2 \mathrm{~s}$, and (e) and (f) $\Delta t_{c t r l}=0.1 \mathrm{~s}$. 
They are plotted top-down in increasing rapidity of control action, and comparing the effects from rapid-pitching presented on the left side directly to rapid flap-actuation depicted on the right side. That is, control action time span, $\Delta t_{c t r l}$ is the same for panels (a) \& (b), (c) \& (d), and (e) \& (f). In spite of the fact that a relatively small amount of pitching $\left(\theta_{p_{c t r l}}=-0.2^{\circ}\right)$ is compared to the limit of nose-up flap-actuation $\left(\beta_{c t r l}=5^{\circ}\right)$, the differences in amplitude of oscillations are noticeable. The observations about the dynamic effects on the rotor as a result of the rapid control action in this configuration are similar to the previous discussion for power reduction (based on figure 3.15), and re-establishes the effectiveness of flap actuation as a rapid control methodology.

A more interesting aspect of these rapid control actions is the power involved in actuation of the control itself, which is designated as $P_{c t r l}$. As discussed in section 2.3, enabling the control action involves overcoming both the inertial loads of the actuation device and the dynamic loads acting on the device. The latter are primarily determined from the instantaneous aerodynamic loads, which depends on various factors such as wind conditions, rotor orientation, and structural deformations. In nominal operating conditions, the NREL 5MW RWT blades are designed to operate with the axis for center of pressure slightly behind the pitching axis (i.e. reference-line of the blade). Consequently, the blades sections operate in a configuration with the tendency to naturally pitch nose-down (i.e. pitching to feather). What this means to pitching as a control action is that energy needs to be dissipated during the process of pitching the blades to feather (positive pitch action), and energy should be supplied in attempting pitching to stall (negative pitch action). Based on 
the configuration for Clark Y trailing-edge flap attached on the NREL 5MW RWT blades, they also have a natural nose-down orientation. This is attributed to the location of flapactuation hinge ahead of the aerodynamic center of the flap section, and hence ensuring the center of pressure will always lie aft of the actuation hinge. Due to the convention differences in pitching and flap-actuation, a natural nose-down tendency for flaps naturally augments a negative flap-actuation control, requiring the system to dissipate energy to effect the actuation in a controlled manner. And in contrast, a positive actuation would require to overcome the natural nose-down alignment and hence would extract energy from the system.

The power needed for control actuation were computed for pitching and flap-actuation for rapid load-control scenarios defined by the control time-span, $\Delta t_{c t r l}=1 \mathrm{~s}, 0.2 \mathrm{~s}$, and $0.1 \mathrm{~s}$. Figure 3.17 shows the instantaneous power required for the control actions, where rapidpitching is juxtaposed with rapid flap-actuation under comparable control scenarios. Each panel depicts the dynamic response for a specific time-span that defines the rapid control scenario, and is shown top-down with an increasing rapidity of control action. The cases shown here are for pitching to feather and nose-down flap-actuation, and correspond to scenarios presented in figure 3.15 effecting the same amount of reduction in power generated by the turbine. From the figure 3.17, it can be observed that with increasing rapidity of control action (or decreasing $\Delta t_{c t r l}$ ), the power required $P_{c t r l}$ increases for both approaches. This increase is more pronounced for pitching action than for flap-actuation, and is associated with the higher inertia of the entire blade as compared to the short-span 

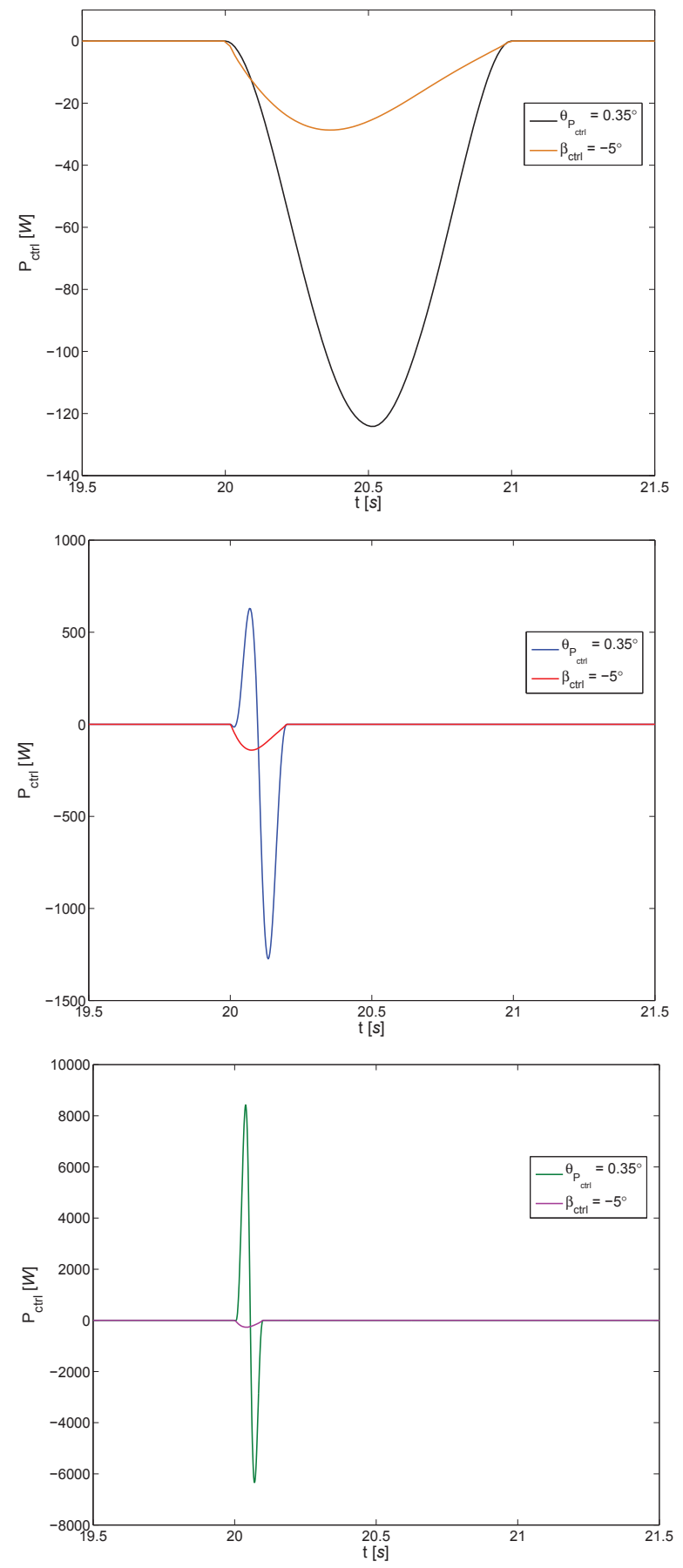

Figure 3.17: Power required to perform rapid control $P_{c t r l}$, plotted in decreasing timescale of the control application. Top panel: $\Delta t_{c t r l}=1 \mathrm{~s}$, middle panel: $\Delta t_{c t r l}=0.2 \mathrm{~s}$, bottom panel: $\Delta t_{c t r l}=0.1 \mathrm{~s}$; each panel compares rapid pitch-control against rapid flapactuation. 
fractional-chord flap. Each of the NREL-5MW RWT blades weigh 17, $740 \mathrm{~kg}$ [74], compared to a meager $28.1 \mathrm{~kg}$ of the flap used along 10\%-span of the blade, in the current design. These inertial differences become significant due to a dependence on the time-span of control action $\Delta t_{c t r l}$. The power required to overcome the aerodynamic moment $M_{a e r}$, and inertial moment $M_{\text {iner }}$ have different dependencies on $\Delta t_{c t r l}$. While the aerodynamic moment remains quasi-constant for the consistent wind scenario that is being studied, the inertial moment depends on the rotational acceleration of the control device. This imposes a dependence for $P_{c t r l}$ only on the rotational velocity of the control actuation to overcome $M_{a e r}$, and an additional dependence on the acceleration of control actuation to overcome $M_{\text {iner. }}$. With increasing rapidity of control actuation the inertial aspect overshadows the aerodynamic one, skewing the power requirement in favor of flap-actuation control that has significantly lesser inertial loads to overcome. This also exposes the limitations of pitching as a rapid load control approach with increasing rapidity, and suggests that trailing-edge flaps are more favorable in such situations.

These observations are further established through visualization of differences between pitching and flap-actuation, presented using peaks of the power required in respective control actions. Figure 3.18 shows the peaks of power involved in rapid-pitching and rapid flap-actuation controls, plotted against increasing time-spans of control actuation for specific cases of $\Delta t_{c t r l}=1 \mathrm{~s}, 0.2 \mathrm{~s}$, and $0.1 \mathrm{~s}$. The solid markers indicate the actual peak powers, irrespective of their application for braking or acceleration, and the solid lines present the respective best-fit curves. The direct comparison cases for power reduction 




Figure 3.18: Peaks powers of control actions, $P_{c t r l}$ for comparable scenarios in rapidpitch and rapid flap-actuation controls, plotted against the control action time span, $\Delta t_{c t r l}$.

would be between $\beta_{c t r l}=-5^{\circ}$ and $\theta_{p_{c t r l}}=0.35^{\circ}$; and for power augmentation would be between $\beta_{c t r l}=5^{\circ}$ and $\theta_{p_{c t r l}}=-0.2^{\circ}$. It is noticeable that with decreasing $\Delta t_{c t r l}$ values, the curves for $\beta_{c t r l}$ show a gradual increase presenting a manageable power requirement for rapid flap-actuation control, whereas the curves for $\theta_{p_{c t r l}}$ depict a drastic increase in the power required for rapid-pitching. 


\section{Chapter 4}

\section{Conclusion}

The study conducted towards this thesis comprised of two main aspects. First, the extension of Common ODE Framework (CODEF) capabilities to evaluate flow-control devices as an active dynamic load control methodology. Second, establishing the limits and effectiveness of contemporary and innovative load control methods through an aeroelastic evaluation of a benchmark turbine under rapid load-control scenarios. This chapter first discusses the fundamental observations and conclusions arrived from the study and later presents a brief outlook of the scope for further research on similar lines.

The control module of the CODEF was added with a functionality to enable the use of flowcontrol devices (FCD) as an active load control technique. The aeroelastic code accepts any 
fractional FCD with the ability to alter the aerodynamics of the rotor without adding significant inertial loads, such as trailing-edge flaps, vortex generators, and retractable microtabs. The code is currently equipped with aerodynamic characteristics for a $20 \%$-chord Clark Y trailing-edge flap attached to either a NACA $64_{3}-618$ or a DU $93-\mathrm{W}-210$. This allows configurations for flap attachments along almost $50 \%$ span of an NREL 5MW RWT blade, which makes up for nearly $75 \%$ of the aerodynamically active part of these blades. This provides a strong foundation to conduct wide range of numerical experiments for the analysis of trailing-edge flaps as flow-control devices on a benchmark turbines such as the RWT.

The oscillating transient behavior observed in the study of pitching as a short-term operational control method suggests several limitations inherent to the aeroelastic response of the rotor. This may preclude the use of conventional pitching as a means to mitigate effects of rapid aerodynamic changes on the rotor such as the ones induced by tower interference, quick gusts, and other similar conditions that occur within a cycle of rotation. There is also the fact that rapid-pitching action may require substantially higher powers of actuation due to the need of imposing a rapid angular accelerations to quickly turn the blade around its own axis. This aspect would become more critical as the size of the state-of-the art turbine increases. This upscaling in rotor size is a definitive trend for reduced cost-of-energy in the envisioned wind-power industry of the future. An increase in blade length results in a higher swept area, squaring the power generation but, there is also a cubical increase in the rotor weight as per the square-cube law (see [94]). This accounts to a bottleneck in 
expansion of load-control techniques that become more energy intensive with increasing inertial loads.

These massive blades associated with larger rotors to be used on future wind super turbines need control systems that are less energy intensive than the pitch actuators. In this respect, innovative control methods such as active flow-control devices (FCDs) are a better alternative. An extensive aeroelastic study conducted with trailing-edge flaps on the benchmark turbine blades indicate capability of such devices to effect global changes in the aeroelastic response of the rotors. Of the scenarios covered, the ability of flaps to reduce instantaneous power generated by actuating in the negative (nose-down) direction is of key interest. These active control devices require low-energy inputs to the actuating mechanisms, and can respond rapidly to dynamic variations in turbine operating conditions. This is significant to effectuating rapid alteration in rotor configuration to mitigate load variations that occur in fraction of a second, and could also be relevant to turbine operation above rated wind speeds. Predominantly, trailing-edge flaps used as active flow-control devices seem like a great alternative to contemporary methodologies in dynamic load control, especially with respect to rapid load variations. Further, they could also be used along with the conventional pitching mechanisms, or with alternative control methods such as variable-speed stall control, and flexo-torsional adaptive blades, to create a hybrid low actuation energy blade that could eventually react fast enough to mitigate the effects of rapidly changing aerodynamic conditions. 


\subsection{Outlook for further work}

To expand the scope of current research, CODEF provides a great platform in furthering the study on active flow-control devices with the benchmark turbine. Some of the avenues identified as needing further exploration are discussed here. Though using trailing-edge flaps on the NREL 5MW RWT standard blade was an effective preliminary study, presence of minor oscillations as the effect of rapid flap-actuation could cause concerns. These fluctuations are attributed to the structural modes of operation of the flexible blades. Future research on these lines should focus on altering the internal structure of the blades enabling swift mitigation of these vibrations or in designing advanced control strategies that consider the presence of these oscillations. The robustness of the control system module and the ability to actuate trailing-edge flaps at very high rates of actuation permit further explorations on this front. 


\section{References}

[1] Siemens and AG, . Press pictures: Direct drive wind turbines for rentel offshore project. 2016. (Accessed July 2017); URL https://www.siemens.com/ press/IM2016120256WPEN.

[2] Siemens AG, . Press pictures: The opening ceremony was a full success. 2016. (Accessed July 2017); URL https://www.siemens.com/press/ IM2016120211WPEN.

[3] Burton, T., Sharpe, D., Jenkins, N., Bossanyi, E.. Wind Energy Handbook. Chichester, UK: Wiley; 2001.

[4] IEC, . Wind turbine generator systems - part 13: Measurement of mechanical loads. Report IEC/TS 61400-13; International Electrotechnical Commission (IEC); 2001.

[5] Griffin, D.A.. Blade system design studies volume I: Composite technologies for large wind turbine blades. Report SAND2002-1879; Sandia National Laboratories; 2002. 
[6] Glauert, H.. The analysis of experimental results in the windmill brake and vortex ring states of an airscrew. Tech. Rep. Reports and Memoranda Volume 1026; Great Britain Aeronautical Research Committee; 1926.

[7] Buhl, M.. A new empirical relationship between thrust coefficient and induction factor for the turbulent windmill state. Tech. Rep.; National Renewable Energy Laboratory; 2005.

[8] Lock, B., Townend, . An extension of the vortex theory of airscrews with applications to airscrews of small pitch, including experimental results. Aeronautical Research Committee, R\&M 1925;1014(1014):1 to 49.

[9] WindEurope, . Wind in power: 2016 european statistics. 2017. (Accessed April 2017); URL https://windeurope.org/about-wind/ statistics/european/wind-in-power-2016/.

[10] Fichaux, N., Beurskens, J., Jensen, P.H., Wilkes, J., Frandsen, S., Sorensen, J., et al. Upwind: Design limits and solutions for very large wind turbines. Sixth Framework Programme 2011;

[11] Madsen, S.F., Bertelsen, K., Krogh, T.H., Erichsen, H.V., Hansen, A.N., Lønbæk, K.B.. Proposal of new zoning concept considering lightning protection of wind turbine blades. Journal of Lightning Research 2012;4(2):108-117. 
[12] Vestas, . Worldś most powerful wind turbine now operational. 2014. (accessed February 2017); URL https://www.vestas.com/en/media/news\#!140128_ nr_vws.

[13] de Vries, E.. Turbines of the year: Offshore turbines. Wind Power Monthly; 2016. (accessed February 2017); URL http://www.windpowermonthly. com/article/1419304/turbines-year-offshore-turbines.

[14] Campbell, S.. 10 of the biggest turbines. Wind Power Monthly; 2016. URL http://www.windpowermonthly.com/10-biggest-turbines.

[15] Manwell, J.F., McGowan, J.G., Rogers, A.L.. Wind energy explained: Theory, design and application. Chichester, UK: Wiley; 2002.

[16] Stuart, J.G., Wright, A.D., Butterfield, C.P.. Wind turbine control systems: dynamic model development using system identification and the fast structural dynamics code. Tech. Rep. NREL/TP-440-22081; National Renewable Energy Laboratory, Golden, CO (United States); 1996.

[17] Barlas, T.K., Van Kuik, G.A.M.. State of the art and prospectives of smart rotor control for wind turbines. In: Journal of Physics: Conference Series; vol. 75. IOP Publishing; 2007, p. 012080.

[18] Wingerden, J.W.V., Hulskamp, A.W., Barlas, T., Marrant, B., Kuik, G.A.M.V., Molenaar, D.P., et al. On the proof of concept of a 'smart'wind turbine rotor blade for load alleviation. Wind Energy 2008;11(3):265-280. 
[19] J. H. Laks, L.Y.P., Wright, A.D.. Control of wind turbines: Past, present, and future. In: 2009 American Control Conference. IEEE; 2009, p. 2096-2103.

[20] Soliman, M., Malik, O.P., Westwick, D.T.. Multiple model predictive control for wind turbines with doubly fed induction generators. IEEE Transactions on Sustainable Energy 2011;2(3):215-225.

[21] Okulov, V.L., Sørensen, J.N.. Optimum operating regimes for the ideal wind turbine. In: Journal of Physics: Conference Series; vol. 75. 2007, p. 012009.

[22] Lobitz, D.W., Veers, P.S.. Load mitigation with bending/twist-coupled blades on rotors using modern control strategies. Wind Energy 2003;6(2):105-117.

[23] Bianchi, F.D., De Battista, H., Mantz, R.J.. Wind turbine control systems: principles, modelling and gain scheduling design. Springer Science \& Business Media; 2006.

[24] Salameh, Z.. Operation of the variable speed constant frequency double output induction generator (vscf-doig) in a constant optimum power coefficient mode. Wind Engineering 1985;9:67-75.

[25] Hohenemser, K.H., Swift, A.H.P.. On the design of horizontal axis two-bladed hinged wind turbines. Journal of Solar Energy Engineering 1984;106(2):171-176. 
[26] Muljadi, E., Pierce, K., Migliore, P.. Control strategy for variable-speed, stallregulated wind turbines. In: Proceedings of the 1998 American Control Conference. ACC (IEEE Cat. No.98CH36207); vol. 3. 1998, p. 1710-1714.

[27] Polinder, H., Bang, D., van Rooij, R.P.J.O.M., McDonald, A.S., Mueller, M.A.. 10 mw wind turbine direct-drive generator design with pitch or active speed stall control. In: 2007 IEEE International Electric Machines \& Drives Conference; vol. 2. 2007, p. $1390-1395$.

[28] Bottasso, C.L., Croce, A., Gualdoni, F., Montinari, P.. Load mitigation for wind turbines by a passive aeroelastic device. Journal of Wind Engineering and Industrial Aerodynamics 2016;148:57-69.

[29] Muljadi, E., Butterfield, C.P.. Pitch-controlled variable-speed wind turbine generation. IEEE Transactions on Industry Applications 2001;37(1):240-246.

[30] Herrera, J.I., Reddoch, T.W., Lawler, J.S.. Harmonics generated by two variable speed wind generating systems. IEEE Transactions on Energy Conversion 1988;3:267-273.

[31] Steinbuch, M.. Optimal multivariable control of a wind turbine with variable speed. Wind Engineering 1987;11(3):153-163.

[32] Bossanyi, E.A.. Further load reductions with individual pitch control. Wind Energy 2005;8(4):481-485. 
[33] Larsen, T.J., Madsen, H.A., Thomsen, K.. Active load reduction using individual pitch, based on local blade flow measurements. Wind Energy 2005;8(1):67-80.

[34] Njiri, J.G., Söffker, D.. State-of-the-art in wind turbine control: Trends and challenges. Renewable and Sustainable Energy Reviews 2016;60:377-393.

[35] Bossanyi, E.A.. Individual blade pitch control for load reduction. Wind Energy 2003;6(2):119-128.

[36] Stol, K.A., Wright, W.Z.A.D.. Individual blade pitch control for the controls advanced research turbine (cart). Journal of Solar Energy Engineering 2006;128(4):498-505.

[37] Johnson, S.J., Baker, J.P., van Dam, C.P., Berg, D.. An overview of active load control techniques for wind turbines with an emphasis on microtabs. Wind Energy 2010;13(2-3):239-253.

[38] Zayas, J., van Dam, C., Chow, R., Baker, J., Mayda, E.. Active aerodynamic load control for wind turbine blades. In: European Wind Energy Conference. Athens, Greece; 2006,.

[39] van Dam, C.P., Berg, D.E., Johnson, S.J.. Active load control techniques for wind turbines. Tech. Rep. SAND2008-4809; Sandia National Laboratories; 2008.

[40] Muljadi, E., Pierce, K.G., Migliore, P.G.. A conservative control strategy for 
variable-speed stall-regulated wind turbines. National Renewable Energy Laboratory; 2000.

[41] Ponta, F.L., Otero, A.D., Rajan, A., Lago, L.I.. The adaptive-blade concept in wind-power applications. Energy for Sustainable Development 2014;22:3-12.

[42] Griffin, D.A.. Evaluation of design concepts for adaptive wind turbine blades. Report SAND2002-2424; Sandia National Laboratories; 2002.

[43] Lago, L.I., Ponta, F.L., Otero, A.D.. Analysis of alternative adaptive geometrical configurations for the NREL-5 MW wind turbine blade. Renewable Energy $2013 ; 59: 13-22$.

[44] Kooijman, H.. Bending-torsion coupling of a wind turbine rotor blade. Netherlands Energy Research Foundation ECN; 1996.

[45] Henriksen, L.C., Bergami, L., Andersen, P.B.. A model based control methodology combining blade pitch and adaptive trailing edge flaps in a common framework. In: European Wind Energy Conference \& Exhibition 2013. 2013,.

[46] Young, A.D.. The aerodynamic characteristics of flaps. Tech. Rep. ARC-R/M-2622; DTIC Document; 1947.

[47] Weyl, A.. High-lift devices and tailless aeroplanes. Aircraft Engineering and Aerospace Technology 1945;17(10):292-297. 
[48] Sadraey, M.H.. Aircraft design: A systems engineering approach. John Wiley \& Sons; 2012.

[49] Jost, E., Lutz, A.F.T., Krämer, E.. An investigation of unsteady 3 d effects on trailing edge flaps. In: Journal of Physics: Conference Series; vol. 753. 2016, p. 022009.

[50] Wilson, D., Barlas, T., Berg, D., van Kuik, G., Resor, B.. Active aerodynamic blade distributed flap control design proceedure for load reduction on the upwind 5mw wind turbine. In: 48th AIAA Aerospace Sciences Meeting Including the New Horizons Forum and Aerospace Exposition. 2010, p. 254.

[51] Behrens, T., Zhu, W.J.. Feasibility of aerodynamic flap hinge moment measurements as input for load alleviation control. EWEA 2011 2011;

[52] Barlas, T.K., van Der Veen, G.J., van Kuik, G.A.M.. Model predictive control for wind turbines with distributed active flaps: incorporating inflow signals and actuator constraints. Wind Energy 2012;15(5):757-771.

[53] Castaignet, D., Barlas, T., Buhl, T., Poulsen, N.K., Wedel-Heinen, J.J., Olesen, N.A., et al. Full-scale test of trailing edge flaps on a vestas v27 wind turbine: active load reduction and system identification. Wind Energy 2014;17(4):549-564.

[54] Migliore, P.G., Miller, L.S., Quandt, G.A.. Wind turbine trailing edge aerodynamic brakes. Tech. Rep. NREL/TP-441-7805; National Renewable Energy Laboratory, Golden, CO (United States); 1995. 
[55] Miller, S., Migliore, P.G., Quandt, G.A.. An evaluation of several wind turbine trailing-edge aerodynamic brakes. Journal of solar energy engineering 1996;118(4):198-203.

[56] Miller, S.L.. Experimental investigation of aerodynamic devices for wind turbine rotational speed control: Phase ii. Tech. Rep. NREL/TP-441-20507; National Renewable Energy Laboratory, Golden, CO (United States); 1996.

[57] Miller, L.S., Huang, S., Quandt, G.. Atmospheric tests of trailing-edge aerodynamic devices. Tech. Rep. NREL/SR-500-22350; National Renewable Energy Laboratory, Golden, CO (United States); 1998.

[58] Stuart, J.G., Wright, A.D., Butterfield, C.P.. Considerations for an integrated wind turbine controls capability at the national wind technology center: an aileron control case study for power regulation and load mitigation. Tech. Rep. NREL/TP-44021335; National Renewable Energy Laboratory, Golden, CO (United States); 1996.

[59] Cahill, J.F.. Summary of section data on trailing-edge high-lift devices. Tech. Rep. NACA-TR-938; National Advisory Committee for Aeronautics(NACA); 1949.

[60] Enenk1, B., Klöppel, V., Preißler, D., Jänker, P.. Full scale rotor with piezoelectric actuated blade flaps. In: 28th European Rotorcraft Forum. 2002, p. 17-19.

[61] Platt, R.C.. Aerodynamic characteristics of wings with cambered external-airfoil flaps including lateral control with a full-span flap. Report NACA-TR-541; National 
Advisory Committee for Aeronautics. Langley Aeronautical Lab, Langley Field VA, United States; 1936.

[62] Platt, R.C., Abbott, I.H.. Aerodynamic Characteristics of NACA 23012 and 23021 Airfoils with 20-percent-chord External-airfoil Flaps of NACA 23012 Section. Defense Technical Information Center; 1936.

[63] Platt, R.C., Shortal, J.A.. Wind-Tunnel Investigation of Wings with Ordinary Ailerons and Full-Span External-Airfoil Flaps. National Advisory Committee for Aeronautics; 1937.

[64] Narsipur, S., Pomeroy, B., Selig, M.. CFD analysis of multielement airfoils for wind turbines. In: Proceedings of the 30th AIAA Applied Aerodynamics Conference. 2012 ,

[65] Hansen, M.O.L., Sørensen, J.N., Vousitas, S., Sørensen, N., Madsen, H.A.. State of the art in wind turbine aerodynamics and aeroelasticity. Prog in Aerospace Sciences $2006 ; 42: 285-330$.

[66] Jonkman, J.M., Buhl Jr, M.L.. Fast user's guide. Tech. Rep. NREL/EL-500-38230; National Renewable Energy Laboratory (NREL); Golden, Colorado, USA; 2005.

[67] Moriarty, P., Hansen, A., (US), N.R.E.L., Engineering, W.. Aerodyn theory manual. National Renewable Energy Laboratory; 2005. 
[68] Laino, D., Hansen, A.. User's guide to the wind turbine aerodynamics computer software aerodyn. Tech. Rep.; National Renewable Energy Laboratory under subcontract No. TCX-9-29209-01; 2002.

[69] Otero, A.D., Ponta, F.L.. Structural analysis of wind-turbine blades by a generalized Timoshenko beam model. Journal of Solar Energy Engineering 2010;132:011015.

[70] Ponta, F.L., Otero, A.D., Lago, L.I., Rajan, A.. Effects of rotor deformation in wind-turbine performance: The dynamic rotor deformation blade element momentum model (drd-bem). Renewable Energy 2016;92:157-170.

[71] Yu, W., Hodges, D.H.. Generalized Timoshenko theory of the variational asymptotic beam sectional analysis. J American Helicopter Society 2005;50:46-55.

[72] Yu, W., Hodges, D.H., Volovoi, V., Cesnik, C.E.S.. On Timoshenko-like modeling of initially curved and twisted composite beams. Int J Sol and Struct 2002;39:51015121.

[73] Hodges, D.H.. Nonlinear Composite Beam Theory. Reston, Virginia: AIAA; 2006.

[74] Jonkman, J., Butterfield, S., Musial, W., Scott, G.. Definition of a 5-MW reference wind turbine for offshore system development. Tech. Rep. NREL/TP-500-38060; National Renewable Energy Laboratory; 2009.

[75] Jamieson, P.. Innovation in wind turbine design. Wiley; 2011. 
[76] Crawford, C.. Re-examining the precepts of the blade element momentum theory for coning rotors. Wind Energy 2006;9(5):457-478.

[77] Crawford, C., Platts, J.. Updating and optimization of a coning rotor concept. Journal of Solar Energy Engineering 2008;130:031002.

[78] Burden, R.L., Faires, J.D.. Numerical analysis. Brooks Cole; 1998.

[79] Mathews, J.H., Fink, K.D.. Numerical methods using Matlab. Prentice Hall; 1999.

[80] Du, Z., Selig, M.S.. A 3-D stall-delay model for horizontal axis wind turbine performance prediction. In: AIAA, Aerospace Sciences Meeting and Exhibit 36th, and 1998 ASME Wind Energy Symposium, Reno, Nevada, USA. American Institute of Aeronautics and Astronautics, ASME International; 1998, p. 9-19.

[81] Eggers, A.J.. Modeling of yawing and furling behavior of small wind turbines. In: 2000 ASME Wind Energy Symposium, 19 th, AIAA, Aerospace Sciences Meeting and Exhibit, 38 th, Reno, NV. 2000, p. 1-11.

[82] Leishman, J.G., Beddoes, T.S.. A generalised model for airfoil unsteady aerodynamic behaviour and dynamic stall using the indicial method. In: 42nd. Annual Forum of the American Helicopter Society, Washington D. C. 1986,

[83] Leishman, J.G., Beddoes, T.S.. A semi-empirical model for dynamic stall. Journal of the American Helicopter Society 1989;34(3):3-17. 
[84] Leishman, J., Beddoes, T.. A generalized model for unsteady aerodynamic behaviour and dynamic stall using the indicial method. Journal of the American Helicopter Society 1990;36:14-24.

[85] Viterna, L.A., Janetzke, D.C.. Theoretical and experimental power from large horizontal-axis wind turbines. Tech. Rep.; National Aeronautics and Space Administration, Cleveland, OH (USA). Lewis Research Center; 1982.

[86] Glauert, H.. Airplane propellers. In: Aerodynamic theory. Springer; 1935, p. 169360.

[87] Wilson, R.E.. Aerodynamic behavior of wind turbines. In: Spera, D., editor. Wind Turbine Technology, Fundamental Concepts of Wind Turbine Engineering. ASME Press, New York; 1994, p. 215-282.

[88] Bak, C., Aagaard Madsen, H., Johansen, J.. Influence from blade-tower interaction on fatigue loads and dynamic (poster). In: Wind energy for the new millennium. Proceedings. 2001 European wind energy conference and exhibition (EWEC'01). 2001, p. $2-6$.

[89] Powles, S.R.J.. The effects of tower shadow on the dynamics of a horizontal-axis wind turbine. Wind Engineering 1983;7:26-42.

[90] Menon, M.. On the aerodynamic properties of slotted-flap flow-control devices for wind turbine applications. Master's thesis; Michigan Technological University; 1400 Townsend Drive, Houghton, MI; 2016. 
[91] Duckwitz, D., Shan, M.. Active tower damping and pitch balancing - design, simulation and field test. In: Journal of Physics: Conference Series; vol. 555. 2014,.

[92] Sørensen, P., Hansen, A.D., Rosas, P.A.C.. Wind models for simulation of power fluctuations from wind farms. Journal of Wind Engineering and Industrial Aerodynamics 2002;90(12?15):1381 - 1402. Fifth Asia-Pacific Conference on Wind Engineering.

[93] Eriksson, S., Bernhoff, H., Leijon, M.. Evaluation of different turbine concepts for wind power. Renewable and Sustainable Energy Reviews 2008;12(5):1419 - 1434.

[94] Thresher, R., Schreck, S., Robinson, M., Veers, P.. Wind energy status and future wind engineering challenges. Tech. Rep. NREL/CP-500-43799; National Renewable Energy Lab; 2008. 


\section{Appendix A}

\section{Copyright Agreements}

This appendix contains the copyright statements that allow the re-use of the following figures: $1.1,1.2$, and 2.3

\section{A.1 Siemens press pictures}

Copyright statement for figures 1.1 and 1.2, from Siemens press pictures:

- Siemens press photos may only be used for editorial purposes. All copyrights belong to Siemens AG, Munich/Berlin, unless another copyright is expressly given. Commercial use or sale of the pictures and data, even in electronically manipulated form, 
is prohibited. The photos may be printed free of charge, but in the case of print media we would appreciate a copy for our records. If pictures are used in films or electronic media, brief notification would suffice.

\section{Attribution:}

$\diamond$ Figure 1.1 was reproduced from a press release from Siemens global website, (C) Siemens AG.

$\diamond$ Figure 1.2 was reproduced from a press release from Siemens global website, (C) Siemens AG.

\section{A.2 Sandia report graphics}

Copyright statement for figure 2.3, from Sandia National Laboratories technical report SAND2002-1879:

- Approved for public release; further dissemination unlimited.

\section{Attribution:}

$\diamond$ Figure 2.3 was reproduced from report SAND2002-1879, prepared by Sandia National Laboratories and made available for public use. 